\title{
A CENSUS OF INTRINSIC NARROW ABSORPTION LINES IN THE SPECTRA OF QUASARS AT $z=2-4^{1}$
}

\author{
Toru Misawa, ${ }^{2}$ Jane C. Charlton, $^{2}$ Michael Eracleous, ${ }^{2,3}$ Rajib Ganguly, ${ }^{4}$ David Tytler, ${ }^{5,6}$ \\ David Kirkman, ${ }^{5,6}$ NaO SuZuki, ${ }^{5,6}$ and Dan Lubin ${ }^{5,6}$ \\ Received 2006 August 11; accepted 2007 January 29
}

\begin{abstract}
We use Keck HIRES spectra of 37 optically bright quasars at $z=2-4$ to study narrow absorption lines that are intrinsic to the quasars (intrinsic NALs, produced in gas that is physically associated with the quasar central engine). We identify $150 \mathrm{NAL}$ systems, which contain $124 \mathrm{C}$ Iv, $12 \mathrm{~N}$ v, and $50 \mathrm{Si}$ iv doublets, of which 18 are associated systems (within $5000 \mathrm{~km} \mathrm{~s}^{-1}$ of the quasar redshift). We use partial coverage analysis to separate intrinsic NALs from NALs produced in cosmologically intervening structures. We find 39 candidate intrinsic systems (28 reliable determinations and 11 that are possibly intrinsic). We estimate that $10 \%-17 \%$ of $\mathrm{C}$ Iv systems at blueshifts of $5000-70,000 \mathrm{~km} \mathrm{~s}^{-1}$ relative to quasars are intrinsic. At least $32 \%$ of quasars contain one or more intrinsic C IV NALs. Considering N v and Si Iv doublets showing partial coverage as well, at least $50 \%$ of quasars host intrinsic NALs. This result constrains the solid angle subtended by the absorbers to the background source(s). We identify two families of intrinsic NAL systems, those with strong $\mathrm{N} v$ absorption and those with negligible absorption in $\mathrm{N} \mathrm{v}$ but with partial coverage in the $\mathrm{C}$ iv doublet. We discuss the idea that these two families represent different regions or conditions in accretion disk winds. Of the 26 intrinsic $\mathrm{C}$ IV NAL systems, 13 have detectable low-ionization absorption lines at similar velocities, suggesting that these are two-phase structures in the wind rather than absorbers in the host galaxy. We also compare possible models for quasar outflows, including radiatively accelerated diskdriven winds, magnetocentrifugally accelerated winds, and pressure-driven winds, and we discuss ways of distinguishing between these models observationally.
\end{abstract}

Subject headings: accretion, accretion disks — galaxies: active — quasars: absorption lines — quasars: general

Online material: extended figure sets, machine-readable table

\section{INTRODUCTION}

Quasars and active galactic nuclei (AGNs) are thought to be powered by accretion of matter onto a supermassive black hole. The accretion flow onto the black hole is thought to proceed via an equatorial accretion disk, which provides a mechanism for removing the angular momentum of the infalling matter. Outflows (winds) from such disks appear to be an inseparable part of this process. More specifically, hydromagnetic winds (e.g., Blandford \& Payne 1982; Emmering et al. 1992; Konigl \& Kartje 1994; Everett 2005) provide a potential mechanism for extracting angular momentum from the accreting material, thus allowing accretion to proceed. Accretion disk winds are likely to be responsible for the broad absorption lines (BALs) observed in the spectra of a fraction of quasars (e.g., Murray et al. 1995; Arav et al. 1994; Proga et al. 2000) and may even be the source of the broad emission lines that are the hallmark of all quasars and AGNs (e.g., Chiang \& Murray 1996; Murray \& Chiang 1997). Understanding the quasar outflow mechanisms by testing and

\footnotetext{
${ }^{1}$ The data presented here were obtained at the W. M. Keck Observatory, which is operated as a scientific partnership among the California Institute of Technology, the University of California, and the National Aeronautics and Space Administration. The Observatory was made possible by the generous financial support of the W. M. Keck Foundation.

2 Department of Astronomy and Astrophysics, Pennsylvania State University, University Park, PA 16802.

${ }^{3}$ Center for Gravitational Wave Physics, Pennsylvania State University, University Park, PA 16802.

4 Department of Physics and Astronomy, University of Wyoming, Laramie, WY 82071.

5 Center for Astrophysics and Space Sciences, University of California at San Diego, La Jolla, CA 92093-0424.

${ }^{6}$ Visiting Astronomer, W. M. Keck Observatory, which is a joint facility of the University of California, the California Institute of Technology, and NASA.
}

refining theoretical models is therefore an essential part of our quest to understand the central engines of AGNs and quasars.

Quasar outflows also have quite important consequences for cosmology and galaxy formation and evolution because they provide energy and momentum feedback to the interstellar medium (ISM) of the host galaxy and to the intergalactic medium (IGM). Simulations of galaxy evolution through mergers (Springel et al. 2005) show that AGN feedback heats the ISM and inhibits star formation. Thus, the colors of the resulting galaxies evolve very quickly to the red, in agreement with the observed color distribution of nearby galaxies. In semianalytic models of galaxy assembly (Granato et al. 2004; Scannapieco \& Oh 2004) AGN feedback expels dense gas from the centers of the host galaxies, heating up the IGM (which it also enriches with metals). Because of its low density, the IGM does not cool efficiently and as a result it cannot fall back onto the galaxy and fuel star formation. Thus, feedback brings about an early termination of the assembly of the host galaxy. In this context it is quite important to know the fraction of quasars driving energetic outflows, as well as the energy content of the outflow. The latter quantity can be determined from the velocity, column density, and global covering factor of the outflowing gas. All of these parameters are intimately connected to dynamical models of accretion disk winds and constraining them observationally can hardly be overemphasized. Moreover, the outflowing material may provide a mechanism for enriching the IGM with metals. This idea is made more attractive by recent results that suggest high metallicities in quasar outflows (e.g., Hamann et al. 1997b; Gabel et al. 2006; Petitjean et al. 1994; Tripp et al. 1996; D'Odorico et al. 2004). This question is closely connected to the issues above since the outflow rates and metallicities of quasar winds are at the heart of the matter. 
In the models cited above, the outflow is accelerated by either magnetocentrifugal forces, radiation pressure in lines and continuum, or a combination of the two processes. The flow originates deep inside the potential well of the black hole and can reach terminal speeds of order $10^{4} \mathrm{~km} \mathrm{~s}^{-1}$ or higher. The geometry of the flow differs between a purely radiation-driven and a purely magnetocentrifugal wind. In the former case (and especially at high luminosities) the flow is largely confined to low latitudes and effectively "hugs" the accretion disk. In addition, the numerical simulations of Proga et al. (2000) show that the region above the fast, low-latitude stream develops transient filaments and streams that are denser than the ambient medium. In contrast, in the latter case the flow is nearly cylindrical (or $U$ shaped) and appears somewhat collimated at large radii from the black hole (see, e.g., illustrations in Blandford \& Payne 1982; Konigl \& Kartje 1994). However, the density of the flow drops sharply once the gas has traveled a distance of a few launch radii, with the consequence that the dense part of the flow is concentrated near the equatorial plane (e.g., Everett 2005). A hybrid model, in which radiation pressure and magnetocentrifugal forces are combined, also leads to a similar geometry (Everett 2005). One may expect by analogy with radiation pressure-driven winds that the higher latitude, lower density regions of these winds would also contain transient, dense filaments or streams. Thus, the apparent geometry and kinematics of the flows resulting from these two acceleration mechanisms are fairly similar.

A different family of outflow models includes pressure-driven winds (e.g., Balsara \& Krolik 1993; Krolik \& Kriss 1995, 2001; Chelouche $\&$ Netzer 2005). These are made up of gas that is photoevaporated from the cool, dense torus that is invoked in AGN unification schemes. Thus, the flow originates not very deep inside the black hole potential well and the outflow speed is of order $10^{3} \mathrm{~km} \mathrm{~s}^{-1}$. The outflow is expected to have a multitemperature and multidensity structure and indeed it would be clumpy. The primary difference between this type of model and the accretion disk wind models described above is the terminal speed of the flow.

Blueshifted (or "P Cygni") absorption lines provide a direct probe of quasar accretion disk winds. BALs (widths $>2000 \mathrm{~km} \mathrm{~s}^{-1}$, by definition) have been the traditional means of studying such winds because they can be readily associated with them. This association is motivated by their large widths (often corresponding to velocity spreads of $30,000 \mathrm{~km} \mathrm{~s}^{-1}$ ) and their smooth profiles. However, intrinsic narrow absorption lines (NALs; widths up to $500 \mathrm{~km} \mathrm{~s}^{-1}$; see review by Hamann \& Sabra 2004) that are physically related to the quasars are an alternative and very useful means of studying such outflows. Intrinsic (most often blueshifted) NALs are detected in a significant fraction $(25 \%-50 \%)$ of quasar spectra. These correspond to UV resonance transitions in highly ionized ions, such as $\mathrm{C}$ IV and $\mathrm{N}$ v (e.g., Foltz et al. 1986, hereafter F86; Sargent et al. 1989; Anderson et al. 1987; Young et al. 1982; Steidel \& Sargent 1991; Ganguly et al. 2001, hereafter G01; Vestergaard 2003). Quasar spectra also host an intermediate class of absorption lines, mini-BALs (widths between 500 and $2000 \mathrm{~km} \mathrm{~s}^{-1}$; see Hamann \& Sabra 2004), whose widths and smooth profiles also suggest an origin in outflows. Such absorption lines are also found in Seyfert 1 galaxies (e.g., Crenshaw et al. 1998, 2004; Scott et al. 2004). Studies of intrinsic NALs and mini-BALs are more promising than studies of BALs for two important reasons: first, these lines do not suffer from self-blending, and second, they are found in a wider variety of AGNs, and with greater ubiquity. In the context of the models described above, intrinsic NALs may probe the dense, cool filaments embedded in a hotter outflow. Thus, they may give us access to a different portion, a different phase, or a different line of sight through the outflow than the BALs.

In spite of their potential importance, NALs have not received as much attention as BALs. Thus, we know surprisingly little about the relation between intrinsic NALs, mini-BALs, and BALs and their connection, if any, to the properties of the broad emission lines. This is because it is difficult to distinguish intrinsic NALs from NALs that are not physically related to the quasars (intervening NALs), produced in intervening galaxies, intergalactic clouds, Milky Way gas, or gas in the host galaxies of the quasars. It has been traditionally thought that NALs that fall within $5000 \mathrm{~km} \mathrm{~s}^{-1}$ of quasar emission redshifts (termed "associated" absorption lines or AALs) are physically associated with the quasars because their frequency per unit velocity increases with decreasing velocity offset from the quasar (e.g., Weymann et al. 1979). AALs are common in all types of quasars, although the strongest ones appear preferentially in radio-loud quasar spectra (F86; Anderson et al. 1987). The statistical analysis of Richards et al. (1999) and Richards (2001) suggested that a fraction of $36 \%$ of the NALs with blueshifts from 5000 to $65,000 \mathrm{~km} \mathrm{~s}^{-1}$ from a quasar may also be physically associated with it.

Over the past decade, with the advent of high-dispersion spectroscopy of faint objects, it has become possible to identify individual intrinsic NALs, based primarily on one or both of the following indicators: (1) the dilution of absorption troughs by unocculted light (e.g., Hamann et al. 1997b; Barlow \& Sargent 1997; Ganguly et al. 1999, hereafter G99; Ganguly et al. 2003; Misawa et al. 2003) and (2) time variability of line profiles (e.g., depth, equivalent width, and centroid), within a year in the absorber's rest frame (e.g., Barlow \& Sargent 1997; Wampler et al. 1995; Hamann et al. 1997a; Wise et al. 2004; Narayanan et al. 2004; Misawa et al. 2005, hereafter M05).

In this paper we use the former of the above techniques to identify intrinsic $\mathrm{C}$ IV, Si Iv, and N v NALs in the high-resolution spectra of 37 quasars at $z_{\mathrm{em}}=2-4$. These quasars were originally selected without regard to NAL properties, although BAL quasars were avoided, and there was a preference for optically bright quasars. These data allow us to construct a large and relatively unbiased sample of intrinsic NALs. Using this sample, we investigate the NAL properties statistically and compare them with the properties of the quasars that host them. Since our spectra typically cover several transitions of the same system, we are also able to probe the ionization state of the absorber. This large sample represents a major advance over most previous efforts, which either dealt with small samples of intrinsic NALs or employed low-resolution spectra in statistical studies.

In $\S 2$ we describe the properties of the quasar sample and briefly summarize the observations. In $\S 3$ we describe our methodology for identifying intrinsic NALs and evaluating the reliability of this determination. Our results are presented in $\S 4$, and their implications in the context of models for quasar outflows are discussed in $\S 5$. Our conclusions are summarized in $\S 6$. In this paper we use a cosmology with $H_{0}=75 \mathrm{~km} \mathrm{~s}^{-1} \mathrm{Mpc}^{-1}$, $\Omega_{m}=0.3$, and $\Omega_{\Lambda}=0.7$. In the Appendix we present all the detailed information on the NALs we have detected. Included in the Appendix are (1) a table of NAL properties derived from fits to their profiles, (2) diagnostic plots of UV resonance doublets, which form the basis of our intrinsic NAL identification method, (3) comparison plots of profiles of different transitions from the same intrinsic NAL system, and (4) detailed notes on individual intrinsic NAL systems.

\section{QUASAR SAMPLE AND OBSERVATIONS}

The quasars in our sample were originally selected and observed in a survey aimed at measuring the deuterium-to-hydrogen 
TABLE 1

Sample Quasars and Their Properties

\begin{tabular}{|c|c|c|c|c|c|c|c|c|c|c|c|c|}
\hline $\begin{array}{c}\mathrm{QSO}^{\mathrm{a}} \\
\text { (1) }\end{array}$ & $\begin{array}{l}z_{\mathrm{em}} \\
(2)\end{array}$ & $\begin{array}{c}m_{V}^{\mathrm{b}} \\
(\mathrm{mag}) \\
(3)\end{array}$ & $\begin{array}{c}m_{R}{ }^{\mathrm{c}} \\
(\mathrm{mag}) \\
(4)\end{array}$ & $\begin{array}{c}f_{\nu}(4400 \AA)^{\mathrm{d}} \\
(\mathrm{mJy}) \\
(5)\end{array}$ & $\begin{array}{c}f_{\nu}(\operatorname{radio})^{\mathrm{e}} \\
(\mathrm{mJy}) \\
(6)\end{array}$ & $\begin{array}{c}\nu^{\mathrm{f}} \\
(\mathrm{GHz}) \\
(7)\end{array}$ & $\begin{array}{c}f_{\nu}(5 \mathrm{GHz})^{\mathrm{g}} \\
(\mathrm{mJy}) \\
(8)\end{array}$ & $\begin{array}{l}\mathcal{R}^{\mathrm{h}} \\
(9)\end{array}$ & $\begin{array}{l}\mathrm{L} / \mathrm{Q}^{\mathrm{i}} \\
(10)\end{array}$ & $\begin{array}{c}v_{\text {up }}(\mathrm{N} \mathrm{v})^{\mathrm{j}} \\
\left(\mathrm{km} \mathrm{s}^{-1}\right) \\
(11)\end{array}$ & $\begin{array}{c}v_{\text {up }}(\mathrm{Si} \mathrm{IV})^{\mathrm{j}} \\
\left(\mathrm{km} \mathrm{s}^{-1}\right) \\
(12)\end{array}$ & $\begin{array}{c}v_{\text {up }}(\mathrm{C} \mathrm{IV}) \\
\left(\mathrm{km} \mathrm{s}^{-1}\right) \\
(13)\end{array}$ \\
\hline Q0014+8118_............. & 3.387 & $\ldots$ & 16.1 & 0.394 & 692.8 & 1.4 & 182.4 & 463 & $\mathrm{~L}$ & 33519 & -1693 & -33084 \\
\hline Q0054-2824........... & 3.616 & & 17.8 & 0.080 & $<2.5$ & 1.4 & $<0.65$ & $<8.11$ & $\mathrm{Q}$ & 38679 & 3543 & -27903 \\
\hline HE $0130-4021 \ldots \ldots$. & 3.030 & 17.02 & $\ldots$ & 0.235 & 4 & 5.0 & 2.6 & 11.2 & Q & 57894 & 23231 & -8247 \\
\hline Q0336-0143........... & 3.197 & $\ldots$ & 18.8 & 0.034 & 446 & 4.85 & 284 & 8460 & $\mathrm{~L}$ & 60999 & 26440 & -5017 \\
\hline Q0450-1310........... & 2.300 & 16.50 & & 0.424 & $<2.5$ & 1.4 & $<0.72$ & $<1.69$ & Q & 54351 & 19577 & -11915 \\
\hline Q0636+6801 ............. & 3.178 & $\ldots$ & 16.9 & 0.194 & 499 & 4.85 & 318 & 1643 & $\mathrm{~L}$ & 68059 & 33768 & 2386 \\
\hline Q0642+4454 ............ & 3.408 & $\ldots$ & 18.4 & 0.047 & 1204 & 4.85 & 755.3 & 16003 & $\mathrm{~L}$ & 46303 & 11318 & -20172 \\
\hline Q0805+0441 …........ & 2.880 & 18.16 & $\ldots$ & 0.084 & 401 & 4.85 & 261 & 3115 & $\mathrm{~L}$ & 74304 & 40285 & 9000 \\
\hline Q0831+1248 ............ & 2.734 & 18.10 & $\ldots$ & 0.091 & $<2.5$ & 1.4 & $<0.69$ & $<7.62$ & Q & 84998 & 51520 & 20478 \\
\hline HE $0940-1050 \ldots \ldots .$. & 3.080 & 16.90 & $\ldots$ & 0.260 & $<2.5$ & 1.4 & $<0.67$ & $<2.58$ & Q & 52404 & 17574 & -13922 \\
\hline Q1107+4847............ & 3.000 & 16.60 & $\ldots$ & 0.347 & $<2.5$ & 1.4 & $<0.68$ & $<1.95$ & $\mathrm{Q}$ & 64739 & 30317 & -1106 \\
\hline Q1157+3143 ........... & 2.992 & 17.00 & $\ldots$ & 0.240 & $<2.5$ & 1.4 & $<0.68$ & $<2.82$ & Q & 66236 & 31872 & 466 \\
\hline $\mathrm{Q} 1208+1011^{\mathrm{k}} \ldots \ldots \ldots$ & 3.803 & & 17.2 & 0.040 & $<2.5$ & 1.4 & $<0.64$ & $<16.0$ & Q & 10885 & -24409 & -55348 \\
\hline Q1244+1129............ & 2.960 & 17.70 & $\ldots$ & 0.127 & $<2.5$ & 1.4 & $<0.68$ & $<5.36$ & Q & -1578 & -36745 & -67293 \\
\hline Q1251+3644 ............ & 2.988 & 19.00 & $\ldots$ & 0.038 & $<2.5$ & 1.4 & $<0.68$ & $<17.8$ & Q & 66522 & 32169 & 767 \\
\hline Q1330+0108 ........... & 3.510 & $\ldots$ & 18.56 & 0.040 & $<2.5$ & 1.4 & $<0.65$ & $<16.2$ & Q & 42793 & 7733 & -23742 \\
\hline Q1334-0033 ......... & 2.801 & 17.30 & $\ldots$ & 0.187 & $<2.5$ & 1.4 & $<0.69$ & $<3.67$ & Q & 79164 & 45379 & 14193 \\
\hline Q1337+2832 ........... & 2.537 & 19.30 & $\ldots$ & 0.031 & $<2.5$ & 1.4 & $<0.70$ & $<25.7$ & $\mathrm{Q}$ & 21637 & -13668 & -44865 \\
\hline $\mathrm{Q} 1422+2309^{1} \ldots \ldots \ldots \ldots$ & 3.611 & $\ldots$ & 15.3 & 0.052 & 503 & 4.85 & 20.2 & 389 & $\mathrm{~L}$ & 23565 & -11732 & -42967 \\
\hline Q1425+6039 ............... & 3.165 & $\ldots$ & 16.0 & 0.449 & $<2.5$ & 1.4 & $<0.67$ & $<1.50$ & $\mathrm{Q}$ & 53082 & 18272 & -13224 \\
\hline Q1442+2931 ........... & 2.670 & 16.20 & $\ldots$ & 0.526 & $<2.5$ & 1.4 & $<0.69$ & $<1.32$ & Q & 89302 & 56071 & 25152 \\
\hline Q1526+6701 ........... & 3.020 & 17.20 & $\ldots$ & 0.199 & 417 & 4.85 & 269 & 1350 & $\mathrm{~L}$ & -4 & -35193 & -65796 \\
\hline Q1548+0917 ............ & 2.749 & 18.00 & $\ldots$ & 0.099 & $<2.5$ & 1.4 & $<0.69$ & $<6.96$ & Q & 83443 & 49881 & 18797 \\
\hline
\end{tabular}

a Quasar names are based on B1950.0 coordinates.

b $V$ magnitude from Véron-Cetty \& Véron (2003).

${ }^{c} R$ magnitude from the USNO-A2.0 Catalog (Monet et al. 1998), except for Q1330+0108, whose $R$ magnitude comes from the USNO-B Catalog ( Monet et al. 2003).

d Optical flux density at $4400 \AA$ in the quasar rest frame (converted from observed flux assuming $f_{\nu} \propto \nu^{-0.44}$; see $\S 2$ ). The flux densities of Q0241-0146 and Q1055+4611 were corrected for Ly $\alpha$ forest contamination as described in $\S 2$.

e Observed radio flux density from NVSS (1.4 GHz; Condon et al. 1998), Griffith et al. (1995; $4.85 \mathrm{GHz})$, or Becker et al. (1991; 4.85 GHz). If no radio source is detected within $10^{\prime \prime}$ of the optical source, we use the detection limit of the survey as an upper limit to the radio flux. The radio fluxes of HE $0130-4021$ and Q1759+7539 come from Smith \& Wright (1980) and Hook et al. (1996), respectively.

${ }^{\mathrm{f}}$ Radio frequency corresponding to the observed radio flux density.

${ }^{\mathrm{g}}$ Radio flux density at $5 \mathrm{GHz}$ in the quasar rest frame, obtained from the observed flux, assuming that $f_{\nu} \propto \nu^{0.7}$.

${ }^{\mathrm{h}}$ Radio loudness parameter, defined in $\S 2$.

i Radio-loud or radio-quiet quasar (see $\S 2$ for definition).

${ }^{j}$ Red limit of the velocity window in which we searched for C IV, N v, or Si Iv NALs.

${ }^{\mathrm{k}}$ This lensed quasar is amplified by a factor of $\sim 3.1$ (Barvainis \& Ivison 2002).

${ }^{1}$ This lensed quasar is amplified by a factor of 15.38 (Kormann et al. 1994).

abundance ratio $(\mathrm{D} / \mathrm{H})$ in the $\mathrm{Ly} \alpha$ forest. The typical value of $\mathrm{D} / \mathrm{H}$ is so small, $(2-4) \times 10^{-5}$ (O'Meara et al. 2001 and references therein), that we can detect only $\mathrm{D}_{\mathrm{I}}$ lines corresponding to $\mathrm{H}_{\mathrm{I}}$ lines with large column densities, $\log \left(N_{\mathrm{H}_{\mathrm{I}}} / \mathrm{cm}^{-2}\right) \geq 16.5$. Therefore, the survey included 40 quasars, in which either damped Ly $\alpha$ (DLA) systems or strong Lyman limit systems (LLSs) were detected. The observations were carried out with Keck HIRES through a $1.14^{\prime \prime}$ slit resulting in a velocity resolution of $\sim 8 \mathrm{~km} \mathrm{~s}^{-1}$ (FWHM). The spectra were extracted by the automated program,
MAKEE, written by Tom Barlow. In this paper we use the spectra of 37 of these quasars, listed in Table 1, which cover the restframe wavelength range between the $\mathrm{Ly} \alpha$ and $\mathrm{C}$ iv lines. This target selection method does not directly bias our sample with respect to the properties of any intrinsic absorption-line systems that these spectra may contain. However, an indirect bias could result since the sample contains only quasars bright enough to allow high signal-to-noise ratio $(\mathrm{S} / \mathrm{N})$, high-resolution observations. Although optical brightness does not appear to be the most 
significant factor in determining whether a quasar hosts a NAL, it is likely to have some effect (G01). Also, there is a somewhat enhanced probability of finding associated NALs in quasars that host BALs (G01); thus, the avoidance of BAL quasars in our sample may bias against intrinsic NALs. We discuss these possible biases, as well as comparisons to other samples selected by different criteria, in $\S 4.1$.

Quasar emission redshifts were obtained from a variety of sources in the literature and are based primarily on measurements of the peaks of their strong, broad UV emission lines, namely, Ly $\alpha, \mathrm{Si}$ IV, and $\mathrm{C}$ IV. It is well known that the redshift determined from these particular lines is systematically different from the redshift of the low-ionization lines (i.e., $\mathrm{Mg}$ II and the Balmer lines) and the systemic redshift, as given by the narrow, forbidden lines (see, e.g., Corbin 1990; Tytler \& Fan 1992; Brotherton et al. 1994; Sulentic et al. 1995; Marziani et al. 1996). Even though redshift differences can reach $\sim 4000 \mathrm{~km} \mathrm{~s}^{-1}$, in most cases they are within $\pm 1000 \mathrm{~km} \mathrm{~s}^{-1}$. In radio-quiet quasars, there is a systematic tendency for the C Iv line to be blueshifted relative to the systemic redshift. Most relevant to this work are the results of Tytler \& Fan (1992), who study the redshift discrepancies of the UV lines in quasars of comparable redshifts and luminosities to those of our sample. They find a mean blueshift of the UV lines relative to the systemic redshift of $260 \mathrm{~km} \mathrm{~s}^{-1}$ and that $90 \%$ of the blueshifts are between 0 and $650 \mathrm{~km} \mathrm{~s}^{-1}$ (see their Fig. 13).

We also list in Table 1 a measure of the radio loudness of the quasars in our sample, the ratio of the flux densities at $5 \mathrm{GHz}$ and $4400 \AA$, i.e., $\mathcal{R}=f_{\nu}(5 \mathrm{GHz}) / f_{\nu}(4400 \AA$ ) (following Kellermann et al. 1989,1994$)$. We adopt $\mathcal{R} \geq 23$ as the criterion for radio loudness (instead of the $\mathcal{R} \geq 10$ adopted by Kellermann et al. $1989,1994)$ in order to separate cleanly the low-luminosity radio sources in our sample from the high-luminosity ones, which have $\mathcal{R}>252$. We derived $f_{\nu}(4400 \AA)$ from the $V$ or $R$ magnitude using the equations (Schmidt \& Green 1983; Oke \& Schild 1970)

$$
\begin{gathered}
m_{V}=-2.5 \log f_{\nu}(5500)-48.60, \\
m_{R}=-2.5 \log f_{\nu}(6600)-48.82
\end{gathered}
$$

and assuming an optical spectral index of $\alpha_{o}=0.44$ (where $f_{\nu} \propto \nu^{-\alpha}$; see Vanden Berk et al. 2001). For Q0241-0146 $\left(z_{\mathrm{em}}=4.040\right)$ and Q1055+4611 $\left(z_{\mathrm{em}}=4.118\right)$, the observed optical fluxes obtained from the $V$ magnitude are underestimated because of contamination by the Ly $\alpha$ forest at $z_{\text {abs }} \sim 3.5$. Therefore, we boosted these fluxes by dividing by the normalized transmission in the $\operatorname{Ly} \alpha$ forest at redshift $z$,

$$
T=\exp \left[-0.0037(1+z)^{3.46}\right]
$$

(Press et al. 1993). The value of $f_{\nu}(5 \mathrm{GHz})$ was obtained from measurements of the flux density at various radio frequencies, assuming a radio spectral index of $\alpha_{r}=0.7$. Thus, our 37 quasars are separated into 12 radio-loud quasars and 25 radio-quiet quasars. Unfortunately, the number of radio-loud quasars that cover the necessary wavelength regions for our analysis is too small to allow a useful statistical study of differences in NAL properties between subclasses.

The properties of the quasars in our sample are summarized in Table 1 and Figure 1. Columns (1) and (2) of Table 1 give the quasar name and emission redshift, and columns (3) and (4) the $V$ and $R$ magnitudes. The optical flux density at $4400 \AA$ in the rest frame is given in column (5). Column (6) lists the observed radio

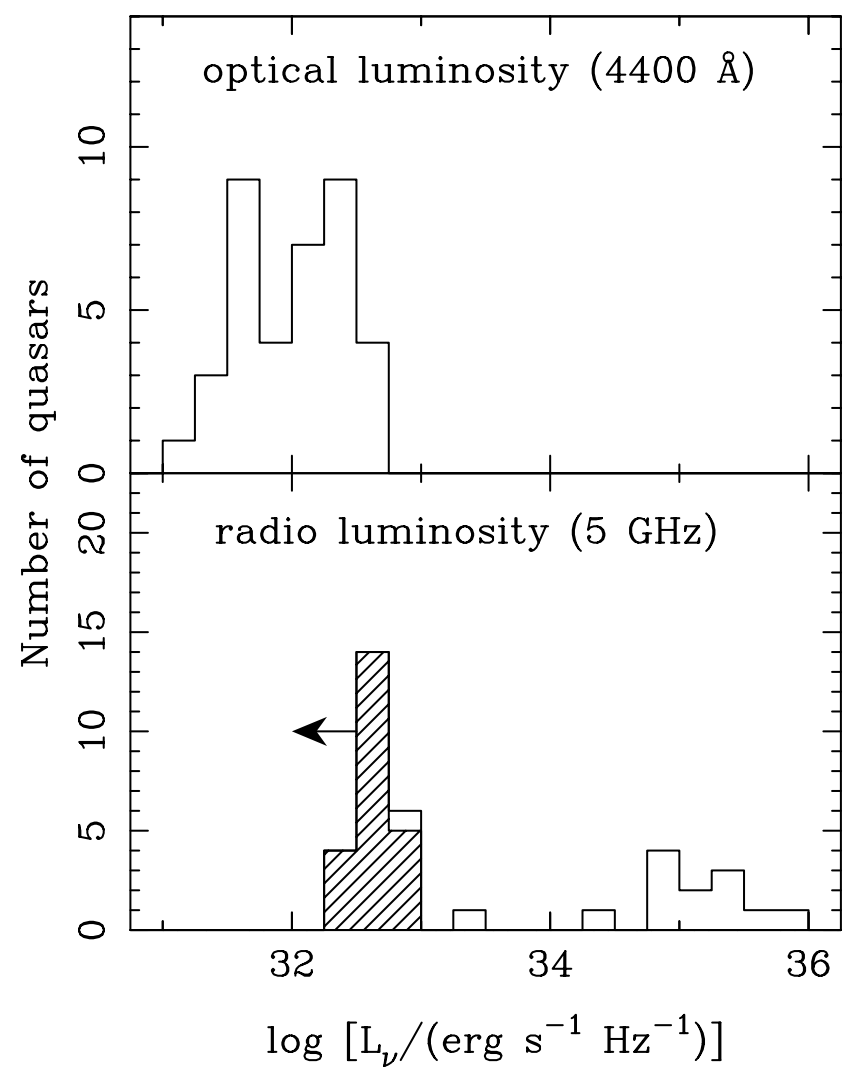

FIG. 1.-Distributions of optical (4400 $\AA$ ) and radio $(5 \mathrm{GHz})$ luminosities of the 37 quasars in our sample. The hatched bins in the bottom panel correspond to upper limits to the radio flux, as listed in Table 1. Radio-loud objects cluster at $5 \mathrm{GHz}$ luminosities between $10^{35}$ and $10^{36} \mathrm{ergs} \mathrm{s}^{-1} \mathrm{~Hz}^{-1}$.

flux at the frequency of column (7), if a radio source was detected within $10^{\prime \prime}$ of the optical source (e.g., Kellermann et al. 1989). The derived radio flux at $5 \mathrm{GHz}$ is listed in column (8). Column (9) gives the radio-loudness parameter, $\mathcal{R}$, based on which the quasars are labeled as L (radio-loud) or Q (radio-quiet) in column (10). The distributions of optical (at $4400 \AA$ ) and radio (at $5 \mathrm{GHz}$ ) luminosities are plotted in Figure 1.

\section{SAMPLE OF NAL SYSTEMS}

To construct our sample of NAL systems for statistical analysis, we first examine the 37 quasar spectra and mark all absorption features that are detected at a confidence level greater than $5 \sigma$, i.e., $\left(1-R_{c, \text { obs }}\right) / \sigma\left(R_{c, \text { obs }}\right) \geq 5\left[R_{c, \text { obs }}\right.$ is the observed residual intensity at the center of a line in the normalized spectrum and $\sigma\left(R_{c, \text { obs }}\right)$ is its uncertainty]. Next, we identify $\mathrm{N}$ v, C Iv, and Si IV doublets in the following regions around the corresponding emission line:

1. $\mathrm{N} v$ absorption doublets: from -5000 to $0 \mathrm{~km} \mathrm{~s}^{-1}$ around the $\mathrm{N} \mathrm{v}$ emission line in the spectra that include this line. ${ }^{7}$ If the spectrum extends redward of the $\mathrm{N} v$ emission line, we also search for absorption doublets up to $+10,000 \mathrm{~km} \mathrm{~s}^{-1}$ to the red of the line. The velocity range is relatively narrow for this transition because of contamination from the Ly $\alpha$ forest, which is rather serious at the redshift of our target quasars.

2. C IV absorption doublets: from $-70,000$ to $0 \mathrm{~km} \mathrm{~s}^{-1}$ around the $\mathrm{C}$ IV emission line in the spectra that include this line. If the spectrum extends redward of the $C$ IV emission line, we

\footnotetext{
7 The velocity offset is defined as negative for NALs that are blueshifted from the quasar.
} 


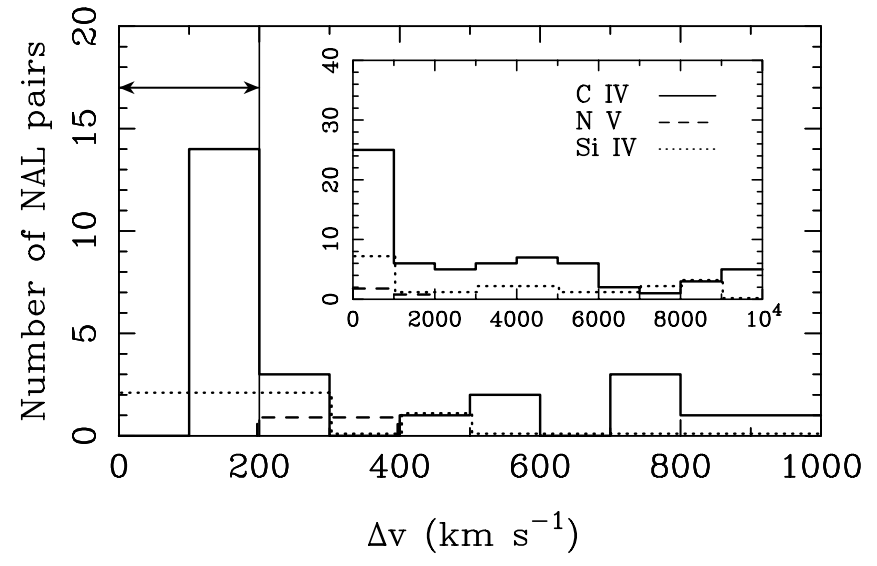

FIG. 2.-Two-point correlation functions for $\mathrm{C}$ IV (solid line), $\mathrm{N} v$ (dashed line), and Si Iv (dotted line) NALs in velocity offset space. Strong clustering is seen at $\Delta v \leq 200 \mathrm{~km} \mathrm{~s}^{-1}$ (indicated by arrows in the main panel), especially for $\mathrm{C}$ IV NALs. Although we count only the number of pairs that are just next to each other, we get a similar result if we count all combinations.

also search for absorption doublets up to $+10,000 \mathrm{~km} \mathrm{~s}^{-1}$ to the red of the line.

3. Si IV absorption doublets: from $-40,000$ to $0 \mathrm{~km} \mathrm{~s}^{-1}$ around the $\mathrm{Si}$ IV emission line in the spectra that include this line. If the spectrum extends redward of the Si IV emission line, we also search for absorption doublets up to $+10,000 \mathrm{~km} \mathrm{~s}^{-1}$ to the red of the line.

In Table 1 we list the red limit of the velocity window that we searched for each quasar.

Absorption troughs that are separated by nonabsorbed regions are considered to be separate lines, even if they are very close to each other. The equivalent width is measured for each separate line by integrating across the absorption profile. In the case of doublets, we represent the equivalent width by that of the stronger (blueward) member. In total, $261 \mathrm{C} \mathrm{IV}, 13 \mathrm{~N} \mathrm{v}$, and $92 \mathrm{Si}$ IV doublets are identified in this manner. To facilitate detailed studies of the systems, we also searched for single metal lines at the same redshifts as the doublet lines even if these were in the Ly $\alpha$ forest. For the statistical analysis, we construct a complete sample that contains only doublet lines whose stronger members would be detected even in our noisiest quasar spectrum. We convert the absorption feature detection criterion given above, $\left(1-R_{c, \text { obs }}\right) / \sigma\left(R_{c, \text { obs }}\right) \geq 5$, to an equivalent width limit using equation (1) of Misawa et al. (2003), and we select absorption features above a certain rest-frame equivalent width. The rest-frame equivalent width limits are set by the spectrum of the quasar Q1330+0108 as follows: $W_{\min }(\mathrm{C}$ IV $)=0.056 \AA$ at $\lambda \approx 5800 \AA, W_{\min }(\mathrm{N} v)=0.038 \AA$ at $\lambda \approx 5700 \AA$, and $W_{\min }(\mathrm{Si}$ IV $)=0.054 \AA$ at $\lambda \approx 5800 \AA$. These limits apply to the stronger member of each doublet. The "homogeneous" NAL sample defined by these limits contains $138 \mathrm{C} \mathrm{IV}, 12 \mathrm{~N}$ v, and $56 \mathrm{Si}$ Iv doublets.

In order to evaluate the column densities $\left(N\right.$ in $\left.\mathrm{cm}^{-2}\right)$ and Doppler parameters $\left(b\right.$ in $\mathrm{km} \mathrm{s}^{-1}$ ) of NALs, we need to deblend the absorption profiles into narrower components. Thus, we used the software package MINFIT (Churchill \& Vogt 2001) to fit absorption lines with Voigt profiles. In the fitting process, we consider the coverage fraction (defined and discussed in detail in $\S 3.1$ ) as a free parameter, as well as the redshift, column density, and Doppler parameter, and we convolve the model with the instrumental profile before comparing with the data. Kinematic components are dropped if a model with fewer components provides an equally acceptable fit to the data. With this
TABLE 2

Census of Poisson Systems, NALs, and Kinematic Components

\begin{tabular}{|c|c|c|c|c|}
\hline $\begin{array}{l}\text { Ion } \\
\text { (1) }\end{array}$ & $\begin{array}{l}\text { Class } \\
(2)\end{array}$ & $\begin{array}{l}\text { Poisson Systems }{ }^{\mathrm{a}} \\
\text { (3) }\end{array}$ & $\begin{array}{l}\text { Lines } \\
(4)\end{array}$ & $\begin{array}{c}\text { Components }^{\mathrm{b}} \\
\text { (5) }\end{array}$ \\
\hline \multirow[t]{5}{*}{ All .................... } & $\mathrm{All}^{\mathrm{c}}$ & 259 & 366 & 871 \\
\hline & Homogeneous $^{\mathrm{d}}$ & 150 & 206 & 706 (313) \\
\hline & $\mathrm{A}^{\mathrm{d}}$ & 28 & 30 & $85(61)$ \\
\hline & $B^{d}$ & 11 & 11 & $47(18)$ \\
\hline & $\mathrm{C}^{\mathrm{d}}$ & 111 & 165 & $574(234)$ \\
\hline \multirow[t]{5}{*}{$\mathrm{C}$ iv ...................... } & $\mathrm{All}^{\mathrm{c}}$ & 224 & 261 & 611 \\
\hline & Homogeneous ${ }^{\mathrm{d}}$ & 124 & 138 & 483 (209) \\
\hline & $\mathrm{A}^{\mathrm{d}}$ & 14 & 14 & $33(20)$ \\
\hline & $B^{d}$ & 9 & 9 & $42(14)$ \\
\hline & $C^{d}$ & 101 & 115 & $408(175)$ \\
\hline \multirow[t]{5}{*}{$\mathrm{N} v$. .................... } & $\mathrm{All}^{\mathrm{c}}$ & 13 & 13 & 42 \\
\hline & Homogeneous $^{\mathrm{d}}$ & 12 & 12 & $41(27)$ \\
\hline & $\mathrm{A}^{\mathrm{d}}$ & 9 & 9 & $31(25)$ \\
\hline & $B^{d}$ & 0 & 0 & $0(0)$ \\
\hline & $\mathrm{C}^{\mathrm{d}}$ & 3 & 3 & $10(2)$ \\
\hline \multirow[t]{5}{*}{ 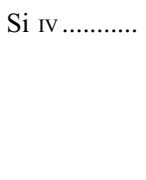 } & $\mathrm{All}^{\mathrm{c}}$ & 72 & 92 & 218 \\
\hline & Homogeneous ${ }^{\mathrm{d}}$ & 50 & 56 & $182(77)$ \\
\hline & $\mathrm{A}^{\mathrm{d}}$ & 7 & 7 & $21(16)$ \\
\hline & $\mathrm{B}^{\mathrm{d}}$ & 2 & 2 & $5(4)$ \\
\hline & $\mathrm{C}^{\mathrm{d}}$ & 41 & 47 & $156(57)$ \\
\hline
\end{tabular}

a Combined multiple NALs that lie within $200 \mathrm{~km} \mathrm{~s}^{-1}$ of each other. See discussion in $\S 3$.

${ }^{\mathrm{b}}$ Narrow kinematic components deblended by Voigt profile fitting with MINFIT. Numbers in parentheses are the number of components whose $C_{f}$ values are physical (i.e., $0<C_{f}$ and $C_{f}<1$ ).

${ }^{c}$ All lines that are detected at greater than a $5 \sigma$ confidence level, $W_{\text {obs }} / \sigma\left(W_{\text {obs }}\right) \geq 5$, regardless of their equivalent width.

${ }^{d}$ Doublets with rest-frame equivalent widths of their stronger (bluer) components larger than the minimum equivalent width that correspond to the $5 \sigma$ detection limit, $W_{\text {min }}$. These limits are $W_{\min }(\mathrm{C}$ Iv $)=0.056 \AA, W_{\min }(\mathrm{N}$ v $)=0.038 \AA$, and $W_{\min }(\mathrm{Si}$ IV $)=0.054 \AA($ see $\S 3)$.

procedure, the NALs of the homogeneous sample are deblended into $483 \mathrm{C}$ IV, $41 \mathrm{~N} \mathrm{v}$, and $182 \mathrm{Si}$ IV components. The observed profiles of $\mathrm{C}$ Iv, $\mathrm{N}$ v, and Si Iv NALs, best-fitting models, and $1 \sigma$ error spectra are shown on a velocity scale relative to the fluxweighted line center in the figures of the Appendix. Almost all the NALs are deblended into multiple components.

To refine our sample for statistical analysis, we combine NALs that lie within $200 \mathrm{~km} \mathrm{~s}^{-1}$ of each other into a single system (a socalled Poisson system). This method is based on the assumption that clustered lines are not physically independent (e.g., Sargent et al. 1988). We chose this clustering velocity after constructing the distribution of velocity separations between C IV NALs, which we show in Figure 2. We note that our adopted clustering velocity differs from the value of $1000 \mathrm{~km} \mathrm{~s}^{-1}$ adopted in previous papers (e.g., Sargent et al. 1988; Steidel 1990; Misawa et al. 2002). We obtain 150 Poisson systems in this manner, of which 124, 12, and 50 systems contain C IV, N v, and Si IV NALs, respectively. All NALs are also classified as AALs and non-AALs. We found 18 associated Poisson systems, of which 9, 12, and 4 systems contain $\mathrm{C}$ IV, N v, and Si Iv NALs, respectively.

Hereafter, we use the terms "(Poisson) system" for a group of NALs within $200 \mathrm{~km} \mathrm{~s}^{-1}$ of each other, "line" or "doublet" for an individual NAL (that may lie within a Poisson system), and "component" for a narrow kinematic component described by a single Voigt profile (as deblended by MINFIT). ${ }^{8}$ In Table 2 we list the total numbers of systems, lines, and components that

\footnotetext{
8 As we noted in $\S 3$, a "line" is separated from its neighbors by nonabsorbed regions.
} 
we have found for each of the three strong absorption lines of interest, namely, $\mathrm{C}$ IV, Si IV, and N v.

We compute the flux-weighted center of a line (or a Poisson system) as the first moment of the line profile, i.e.,

$$
\lambda_{\mathrm{obs}}=\langle\lambda\rangle=\frac{\sum \lambda_{i}\left[1-R\left(\lambda_{i}\right)\right]}{\sum\left[1-R\left(\lambda_{i}\right)\right]},
$$

and the line dispersion, $s$, via

$$
s_{\lambda}=\sqrt{\left\langle\lambda^{2}\right\rangle-\langle\lambda\rangle^{2}}
$$

where $\left\langle\lambda^{2}\right\rangle$ is the second moment of the line profile, given by

$$
\left\langle\lambda^{2}\right\rangle=\frac{\sum \lambda_{i}^{2}\left[1-R\left(\lambda_{i}\right)\right]}{\sum\left[1-R\left(\lambda_{i}\right)\right]} .
$$

The above sums are evaluated over the entire width (full width at zero depth) of a line. The quantity $R\left(\lambda_{i}\right)$ is the intensity at pixel $i$ in the normalized spectrum and $\lambda_{i}$ is the wavelength of that pixel. Using the above quantities, we obtain the flux-weighted line width from

$$
\sigma(v)=\sqrt{2} s_{v}=\sqrt{2}\left(\frac{s_{\lambda}}{\langle\lambda\rangle}\right) c \quad\left(\mathrm{~km} \mathrm{~s}^{-1}\right) .
$$

Using the flux-weighted wavelength of a line, we compute its flux-weighted redshift, and then its velocity offset by the relativistic Doppler formula, i.e.,

$$
\beta \equiv \frac{v_{\mathrm{shift}}}{c}=-\frac{\left(1+z_{\mathrm{em}}\right)^{2}-\left(1+z_{\mathrm{abs}}\right)^{2}}{\left(1+z_{\mathrm{em}}\right)^{2}+\left(1+z_{\mathrm{abs}}\right)^{2}},
$$

where $z_{\mathrm{em}}$ and $z_{\mathrm{abs}}$ are the emission redshift of the quasar and the absorption redshift of the line, respectively. The wavelengths, redshifts, velocity offsets, and flux-weighted widths of detected lines, computed as described above, are given in the Appendix, where we also include NALs that do not meet the rest-frame equivalent width criterion for our homogeneous sample (i.e., they have $\left.W_{\text {rest }}<W_{\min }\right)$.

\subsection{Partial Coverage Analysis}

We identified intrinsic NAL candidates by looking for absorption troughs that were diluted by unocculted light from the background source. The optical depth ratio of doublet lines, such as C IV, Si IV, or N v, sometimes deviates from the value of 2:1 expected from atomic physics. This discrepancy can be explained if the absorber covers the background flux source only partially and the unabsorbed flux changes the relative depth of the lines (e.g., Wampler et al. 1995; Barlow \& Sargent 1997; Hamann et al. 1997b; G99). However, there are other possible explanations, such as local emission by the absorbers (Wampler et al. 1995) and scattering of background photons into our line of sight (G99). We assume the simplest model, in which the absorber has a constant optical depth across the projected area (i.e., a homogeneous model). Then, the observed intensity as a function of velocity from the line center in the normalized spectrum is given by

$$
R(v)=\left[1-C_{f}(v)+C_{f}(v) e^{-\tau(v)}\right],
$$

where $C_{f}(v)$ is the fraction of background light occulted by the absorber (hereafter the "coverage fraction") and $\tau(v)$ is the optical depth at velocity $v$. If a NAL system consists of many kinematic components (the most common case), these are combined by multiplying the individual Voigt profiles after making the appropriate adjustments for partial coverage, following equation (9). Thus, we describe the final normalized residual intensity by the product

$$
R(v)=\prod_{k}\left[1-C_{f, k}(v)+C_{f, k}(v) e^{-\tau_{k}(v)}\right],
$$

where the index $k$ labels different kinematic components. This assumption is quite safe if the components do not overlap significantly in velocity and is still a good approximation if one component dominates at each wavelength.

If we measure the optical depth ratio of doublet lines with oscillator strength values of $2: 1$ (e.g., $\mathrm{C}$ IV, $\mathrm{N} \mathrm{v}$, and Si IV) by fitting their profiles, we can evaluate the coverage fraction as a function of velocity across the line profile as

$$
C_{f}(v)=\frac{\left[1-R_{r}(v)\right]^{2}}{1+R_{b}(v)-2 R_{r}(v)},
$$

where $R_{r}$ and $R_{b}$ are the continuum-normalized intensities of the weaker (redder) and stronger (bluer) members of the doublet (see, e.g., Barlow \& Sargent 1997). G99 refined this technique by considering two background sources: the continuum source and the broad emission line region (BELR). In this composite picture the total coverage fraction can be expressed as a weighted average of the coverage fractions of the two regions, namely,

$$
C_{f}(v)=\frac{C_{c}(v)+w(v) C_{\mathrm{BELR}}(v)}{1+w(v)},
$$

where $C_{c}(v)$ and $C_{\mathrm{BELR}}(v)$ are the coverage fractions of the continuum source and the BELR, respectively, and $w(v)$ is the ratio of the broad-line flux to the continuum flux at a given pixel in the absorption-line profile.

It is quite possible for the absorber to have different optical depths along different paths (i.e., an inhomogeneous model; de Kool et al. 2002). Nonetheless, by exploring this inhomogeneous model, Sabra \& Hamann (2005) found that the average optical depths derived from homogeneous and inhomogeneous models are consistent with each other within a factor of $\leq 1.5$, unless a small fraction of the projected area of the absorber has a very large optical depth. Moreover, in most cases, one can find acceptable fits to the absorption profile using a simple combination of just a few Voigt components. Therefore, we adopt the homogeneous optical depth model, as described by equation (11), with the understanding that $C_{f}$ describes the fraction of all background photons that pass through the absorber. We consider the composite picture described by equation (12), only if it critically affects our conclusions.

We use two methods to evaluate $C_{f}$ : (1) the pixel-by-pixel method (e.g., G99), in which we apply equation (11) to each pixel in the normalized spectrum, and (2) the fitting method (e.g., Ganguly et al. 2003), in which we fit the absorption profiles using MINFIT, treating $C_{f}$, as well as $\log N, b$, and $z_{\text {abs }}$, as a free parameter. In the former case we obtain a value of $C_{f}$ for each pixel in the line profile, while in the latter case we obtain a value of $C_{f}$ for each kinematic (Voigt) component. Our derived $C_{f}$ values are subject to a number of additional uncertainties resulting from line blending, uncertainties in the continuum fit, the convolution of the spectrum with the line-spread function (LSF) of 


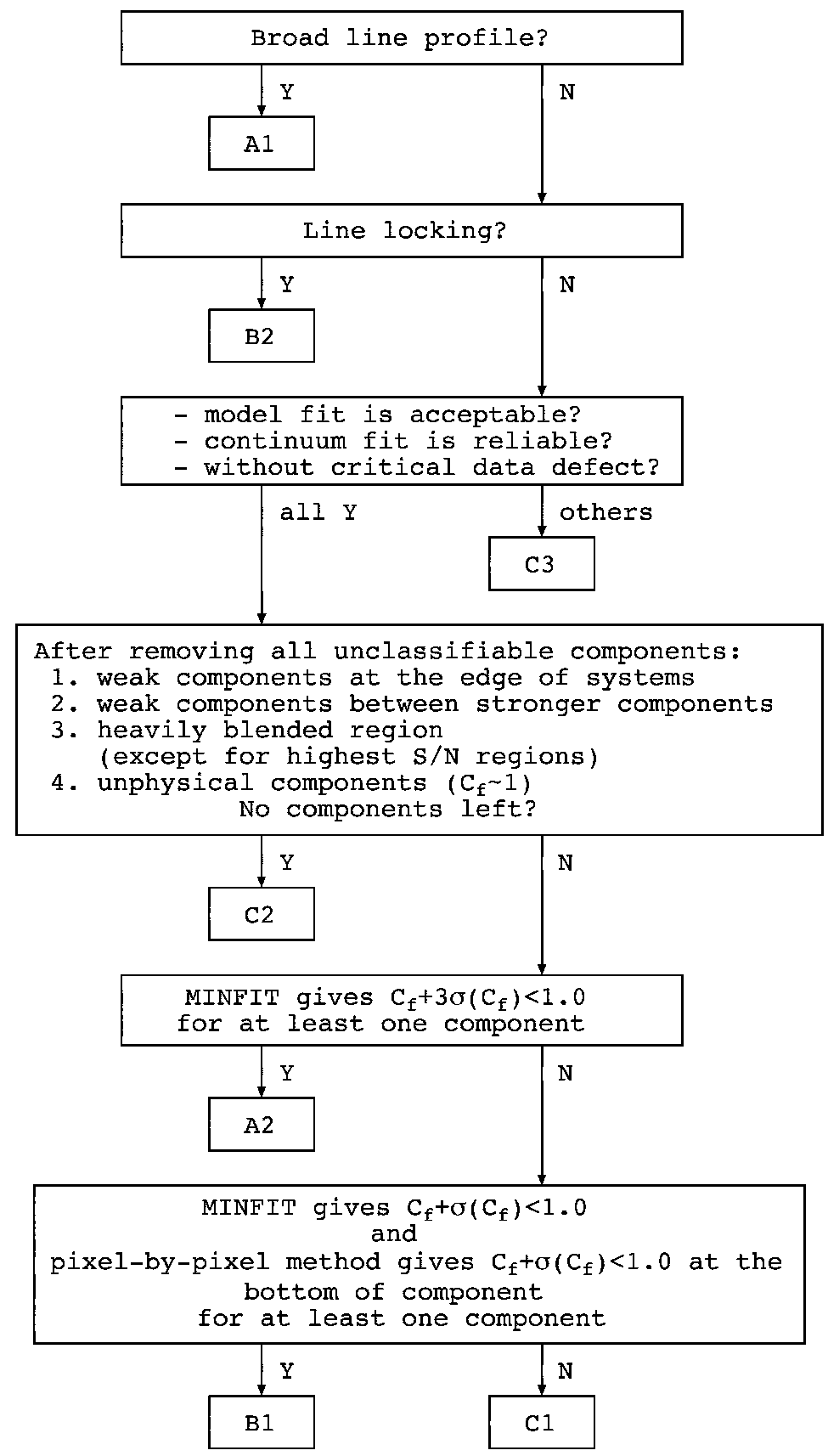

Fig. 3.-Flow chart illustrating our scheme of NAL classification. The classes indicate how reliably we can determine whether the NALs are intrinsic or not, based on partial coverage analysis. A more detailed discussion of this scheme is given in $\S 3.1$.

the spectrograph (G99; only applies to the pixel-by-pixel method), and Poisson errors in regions with weak lines (only applies to the fitting method). The methodology for assessing these uncertainties was developed in M05 with the help of extensive simulations. We do not use spectral regions that are strongly affected by these uncertainties, and we consider these sources of error when evaluating whether $C_{f}$ deviates from unity, as described below. The $C_{f}$ values evaluated by both of the above methods are overplotted on the observed spectrum of each doublet shown in Figure Set 12 in the Appendix.

The MINFIT routine sometimes gives unphysical coverage fractions such as $C_{f}<0$ or $C_{f}>1$. Tests by M05 showed that coverage fractions produced by MINFIT are very sensitive to continuum level errors, especially for very weak components whose $C_{f}$ values are close to 1 , which suggests a cause for these unphysical results. In such cases, other fit parameters, such as the column density and Doppler parameter, have no physical meaning. Therefore, we reevaluate them assuming $C_{f}=1$ (with no error bar) for those components and by refitting the rest of the line.

Based on the results of the coverage fraction analysis, we separate all NALs into three classes based on the reliability of the conclusion that they are intrinsic: classes A and B, respectively, contain "reliable" and "possible" partially covered NALs (i.e., intrinsic NAL candidates), and class C consists of NALs with no evidence for partial coverage (i.e., intervening or unclassified NALs). We may regard classes $\mathrm{A}-\mathrm{C}$ as including progressively 
TABLE 3

Statistical Properties of Poisson Systems of NALs

\begin{tabular}{|c|c|c|c|c|c|c|c|c|c|c|}
\hline \multirow[b]{2}{*}{$\begin{array}{l}\text { SAMPLE } \\
\text { (1) }\end{array}$} & \multirow[b]{2}{*}{$\begin{array}{c}\text { Number/DENSITY } \\
\text { (2) }\end{array}$} & \multicolumn{3}{|c|}{ C IV } & \multicolumn{3}{|c|}{$\mathrm{N} \mathrm{v}$} & \multicolumn{3}{|c|}{ Si IV } \\
\hline & & $\begin{array}{l}\text { AAL } \\
(3)\end{array}$ & $\begin{array}{l}\text { Non-AAL } \\
\text { (4) }\end{array}$ & $\begin{array}{c}\text { Total } \\
(5)\end{array}$ & $\begin{array}{l}\text { AAL } \\
(6)\end{array}$ & $\begin{array}{l}\text { Non-AAL } \\
\text { (7) }\end{array}$ & $\begin{array}{c}\text { Total } \\
(8)\end{array}$ & $\begin{array}{l}\text { AAL } \\
(9)\end{array}$ & $\begin{array}{l}\text { Non-AAL } \\
\text { (10) }\end{array}$ & $\begin{array}{l}\text { Total } \\
(11)\end{array}$ \\
\hline \multirow[t]{2}{*}{ Path length ..................... } & $\delta z^{\mathrm{a}}$ & 0.64 & 17. & 18. & 2.3 & 0.32 & 2.6 & 1.3 & 12. & 14. \\
\hline & $\delta \beta^{\mathrm{a}}$ & 0.17 & 4.9 & 5.1 & 0.58 & 0.079 & 0.66 & 0.32 & 3.3 & 3.6 \\
\hline \multirow[t]{3}{*}{ Class A........................... } & $N^{\mathrm{b}}$ & $3(3)$ & $11(8)$ & $14(9)$ & $9(7)$ & $0(0)$ & $9(7)$ & $0(0)$ & $7(7)$ & $7(7)$ \\
\hline & $d N / d z$ & 4.7 & 0.63 & 0.78 & 3.9 & 0.0 & 3.4 & 0.0 & 0.57 & 0.5 \\
\hline & $d N / d \beta$ & 18. & 2.3 & 2.8 & 16. & 0.0 & 14. & 0.0 & 2.2 & 2.0 \\
\hline \multirow[t]{3}{*}{ Class $\mathrm{A}+\mathrm{B} \ldots \ldots \ldots \ldots \ldots \ldots \ldots \ldots \ldots \ldots \ldots$} & $N^{\mathrm{b}}$ & $3(3)$ & $20(11)$ & $23(12)$ & $9(7)$ & $0(0)$ & $9(7)$ & $0(0)$ & $9(9)$ & $9(9)$ \\
\hline & $d N / d z$ & 4.7 & 1.2 & 1.3 & 3.9 & 0.0 & 3.4 & 0.0 & 0.73 & 0.66 \\
\hline & $d N / d \beta$ & 18. & 4.1 & 4.6 & 16. & 0.0 & 14. & 0.0 & 2.8 & 2.5 \\
\hline \multirow[t]{3}{*}{ 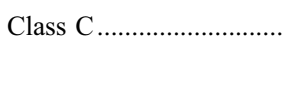 } & $N^{\mathrm{b}}$ & $6(5)$ & $95(34)$ & $101(34)$ & $3(3)$ & $0(0)$ & $3(3)$ & $4(4)$ & 37 (24) & $41(24)$ \\
\hline & $d N / d z$ & 9.4 & 5.5 & 5.6 & 1.3 & 0.0 & 1.1 & 3.2 & 3.0 & 3.0 \\
\hline & $d N / d \beta$ & 36. & 19. & 20. & 5.2 & 0.0 & 4.6 & 13. & 11. & 11. \\
\hline \multirow[t]{3}{*}{ 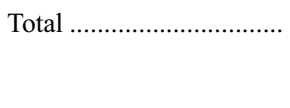 } & $N^{\mathrm{b}}$ & 9 (13) & 115 (37) & 124 (37) & $12(37)$ & $0(0)$ & $12(37)$ & $4(24)$ & $46(37)$ & $50(37)$ \\
\hline & $d N / d z$ & 14. & 6.6 & 6.9 & 5.2 & 0.0 & 4.6 & 3.2 & 3.7 & 3.7 \\
\hline & $d N / d \beta$ & 54. & 24. & 25. & 21. & 0.0 & 18. & 13. & 14. & 14. \\
\hline \multirow[t]{2}{*}{ 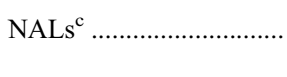 } & A/Total & $33 \%$ & $10 \%$ & $11 \%$ & $75 \%$ & $\ldots$ & $75 \%$ & $0 \%$ & $15 \%$ & $14 \%$ \\
\hline & $\mathrm{A}+\mathrm{B} /$ Total & $33 \%$ & $17 \%$ & $19 \%$ & $75 \%$ & $\ldots$ & $75 \%$ & $0 \%$ & $20 \%$ & $18 \%$ \\
\hline \multirow[t]{2}{*}{ 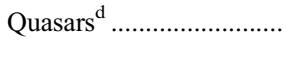 } & A/Total & $23 \%$ & $22 \%$ & $24 \%$ & $19 \%$ & $\ldots$ & $19 \%$ & $0 \%$ & $19 \%$ & $19 \%$ \\
\hline & $\mathrm{A}+\mathrm{B} /$ Total & $23 \%$ & $30 \%$ & $32 \%$ & $19 \%$ & $\ldots$ & $19 \%$ & $0 \%$ & $24 \%$ & $24 \%$ \\
\hline
\end{tabular}

${ }^{\text {a }}$ The total redshift and speed intervals considered in the determination of $d N / d z$ and $d N / d \beta$.

b $N$ denotes the number of Poisson systems (i.e., groups of NALs that lie within $200 \mathrm{~km} \mathrm{~s}^{-1}$ of each other; see $\S 3$ ). The number in parentheses gives the number of quasars in which these Poisson systems are found.

c Percentage of class A and class A+B NALs relative to all NALs, broken down by transition and by velocity window (associated vs. nonassociated).

d Percentage of quasars hosting class A and class A+B NALs relative to all quasars, broken down by transition and by velocity window (associated vs. nonassociated).

smaller fractions of intrinsic NALs, and we treat them as such in our subsequent statistical analysis.

We classified four mini-BALs as class A systems. We also classified three NALs into class B, although they did not exhibit partial coverage because they show line locking, which is often seen in intrinsic NALs at $z_{\mathrm{abs}} \sim z_{\mathrm{em}}$. In the list below and in the flow chart of Figure 3, we give the criteria and method that we used to classify NALs into reliability classes:

1. Class A: Reliable intrinsic NAL candidates:

a) A1: Smooth and broad (self-blended) line profile (i.e., mini-BAL).

b) A2: MINFIT gives $C_{f}+3 \sigma\left(C_{f}\right)<1$ for at least one component.

2. Class B: Possible intrinsic NAL candidates:

a) B1: Both MINFIT and the pixel-by-pixel method give $C_{f}+\sigma\left(C_{f}\right)<1$ at the center of a component for at least one kinematic component.

b) B2: Line locked.

3. Class $\mathrm{C}$ : Lines without partial coverage and unclassifiable lines:

a) $\mathrm{C} 1$ : MINFIT gives $C_{f}+\sigma\left(C_{f}\right) \geq 1$ for all components.

b) C2: No components can be used for classification because they suffer from systematic errors as described in the next paragraph.

c) C3: The system is not acceptable for classification because of problems due to model fit, continuum fit, or critical data defect.

During line classification, we ignore components that satisfy the following criteria. These were established by following the same procedure to fit strong $\mathrm{Mg}$ II doublets identified in the same spectra. For the strong Mg II lines we would expect full coverage for all components, but we find $C_{f}$ to be inconsistent with unity if these criteria apply:

1. Weak components at the edge of a system.

2. Weak components between much stronger components.

3. Components in a heavily blended region (except for regions with extremely high $\mathrm{S} / \mathrm{N}$ ).

4. Components for which we obtained unphysical values of $C_{f}$ in the first fitting trial. As we noted above, we set $C_{f}=1$ for such components and repeat the fit for the other components in the system.

If at least one component of a NAL satisfies one of the criteria for class A or B, we would classify the NAL as an intrinsic NAL candidate, even if the other components are consistent with full coverage. This applies even for a Poisson system for which only one component in one of the separate lines satisfies the class A or B criteria. Also, if a Poisson system has more than one doublet transition detected and one or more of the transitions ( $\mathrm{C}$ IV, N v, or Si IV) belongs in class A, it is considered a class A intrinsic system. Similarly, such a system will be considered a class B system if one or more transitions fall in class B (and none are class A).

Placing a Poisson system in class A or B if only one component satisfies the classification criteria involves the following caveat: the class A or B NAL may be superposed on an intervening system by chance. We have thus evaluated the probability of this chance superposition, using the information in Table 3. For example, we have searched for associated C IV NALs in a velocity range $\delta \beta=0.17$ and found three class $\mathrm{A}+\mathrm{B}$ and six class C NALs. Since each NAL typically spans a velocity window of $200 \mathrm{~km} \mathrm{~s}^{-1}$ or less, the class C NALs cover $2.4 \%$ of this velocity window. Therefore, the probability that any one of the three class A+B NALs would be blended with a class C NAL (i.e., the expected number of blends) is 0.07 . Similarly, we 

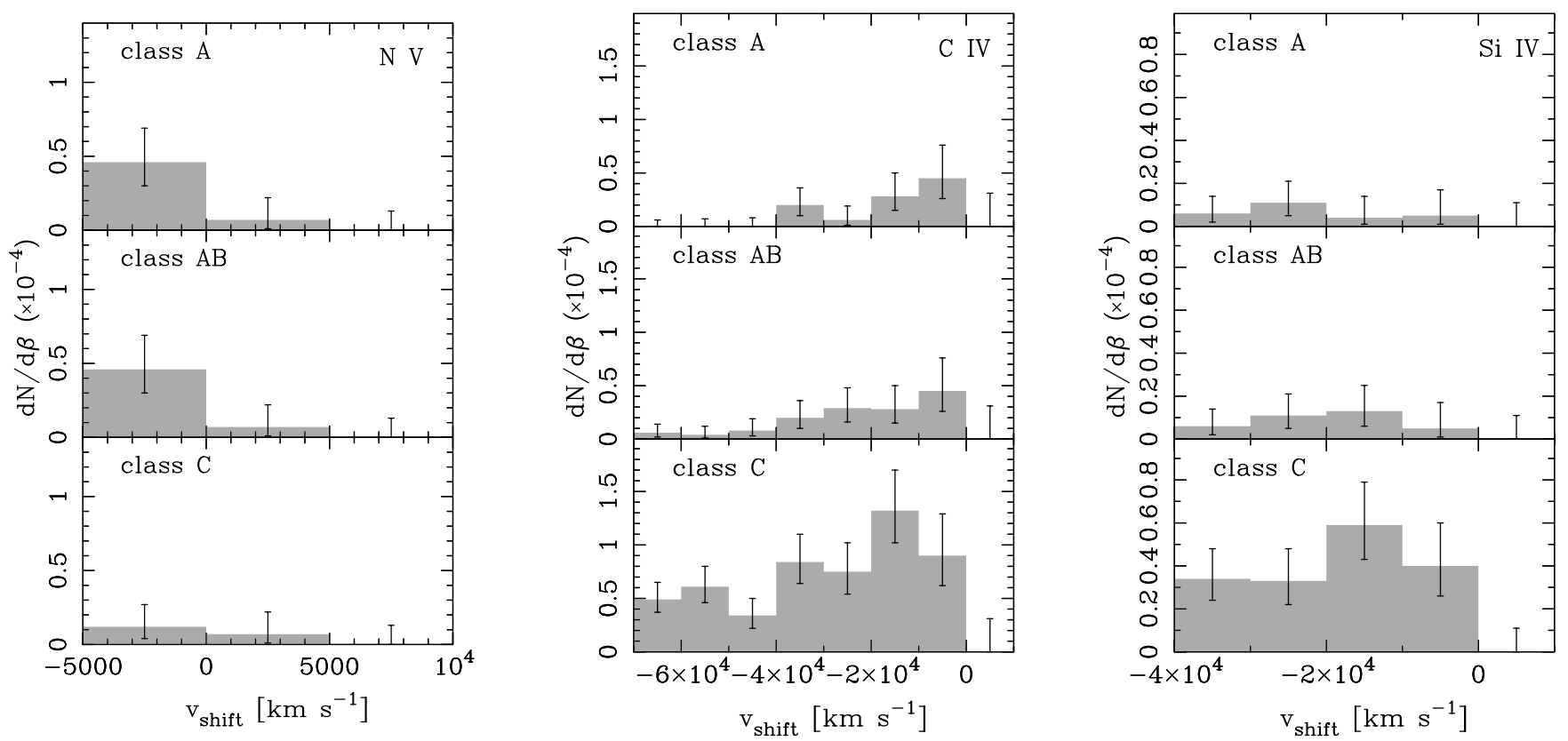

FIg. 4.-Velocity offset distribution of $\mathrm{C}$ Iv, $\mathrm{N}$ v, and $\mathrm{Si}$ Iv systems. For each transition we plot separately the distribution of class A systems (reliable), class A+B systems (reliable+possible), and class C systems (intervening/unclassified).

estimate the number of blends to be 0.25 in the nonassociated $\mathrm{C}$ IV velocity range, 0.03 in the $\mathrm{N} v$ velocity range (associated only), and 0.07 in the Si Iv velocity range (nonassociated only). Thus, we conclude that the danger of a blend is negligible.

In our final sample of 150 Poisson NAL systems, 28 fall into class A, 11 into class B, and 111 into class $C$. The numbers of systems, lines, and components are summarized in Table 2. In the Appendix we list the detailed classification results, along with the subcategory numbers that give the basis for the classification (e.g., A2, C3, . .). In the Appendix we also give detailed comments on individual NAL systems. In Table 2 we give a census of the resulting Poisson systems, NALs, and kinematic components.

\section{RESULTS}

Using the complete NAL sample constructed in the previous section, we first investigate the density of NALs per unit redshift interval and per unit velocity interval (i.e., the velocity offset distribution, $d N / d z$ and $d N / d \beta=c d N / d v$, respectively). Next, we examine the relative numbers of intrinsic NALs in different transitions. This is of interest because these relative numbers could be indicative of the ionization structure of the absorbers and also of their locations relative to the continuum source. Our analysis provides only lower limits on the intrinsic NAL fractions because intrinsic absorbers need not always show partial coverage and because some of our sample quasar spectra cover only very small redshift windows for NAL detection. We also examine the distributions of coverage fractions and line profile widths and consider the ionization conditions of the intrinsic NALs in our sample. Finally, we briefly compare the properties of quasars and the properties of intrinsic NALs.

\subsection{Velocity Offset and Equivalent Width Distribution}

In evaluating $d N / d z$, we exclude segments of spectra in echelle order gaps, which affect about $8.8 \%, 16.0 \%$, and $23.0 \%$ of the spectral regions at $\sim 5500, \sim 6000$, and $\sim 6500 \AA$, respectively. We implicitly assume that all NALs arise in outflows from the quasars, and we use the velocity offset computed by equation (8).
In Table 3 we summarize our derived values of $d N / d z$ and $d N / d \beta$ for different transitions and for different velocity offsets relative to the quasar, and we also break down the results by reliability class. The velocity offset distributions of $\mathrm{C}$ Iv, $\mathrm{N}$ v, and $\mathrm{Si}$ iv NALs are presented in Figure 4, broken down according to reliability class. It is noteworthy that intrinsic NAL candidates (i.e., class A and $\mathrm{B}$ ) are found not only among AALs but also among non-AAL systems.

In previous studies using low-resolution spectra, the velocity offset distributions of C Iv NALs are found to be almost uniform up to $v_{\text {shift }} \sim-70,000 \mathrm{~km} \mathrm{~s}^{-1}$ (the maximum velocity at which the $\mathrm{C}$ IV doublet can be detected without blending with the Ly $\alpha$ forest). However, a significant excess of AALs was found within $5000 \mathrm{~km} \mathrm{~s}^{-1}$ of radio-loud and steep-spectrum quasars (Young et al. 1982; Richards et al. 1999, 2001; F86). Weymann et al. (1979) presented evidence that the distribution of intrinsic C Iv NALs extends up to $v_{\text {shift }} \sim-18,000 \mathrm{~km} \mathrm{~s}^{-1}$. Richards et al. (1999) found an excess of C Iv NALs with $v_{\text {shift }} \gtrsim-65,000 \mathrm{~km} \mathrm{~s}^{-1}$ in optically luminous quasars, in radio-quiet over radio-loud quasars, and in flat radio spectrum quasars over steep radio spectrum quasars. Our results show no remarkable excess of NALs in radioloud quasars, nor any strong excess of NALs near the quasars in either the radio-loud or radio-quiet subsamples. This is not surprising, however, since our subsamples of radio-loud and radioquiet quasars are relatively small and heterogeneous.

Considering all $\mathrm{C}$ iv NALs (from classes $\mathrm{A}, \mathrm{B}$, and $\mathrm{C}$ ) together, we do find (see Table 3 ) that for C iv NALs the values of $d N / d z$ and $d N / d \beta$ in associated regions are about twice as large as those in nonassociated regions, in general agreement with the results of Weymann et al. (1979). In Figure 5 we show the velocity offset distribution of all C Iv NALs from our survey compared to that of class $\mathrm{A}+\mathrm{B}$ NALs.

The distribution of rest-frame equivalent widths of the $\mathrm{C}$ IV Poisson systems detected in our survey is shown in Figure 6. This distribution rises sharply toward our detection limit of $0.056 \AA$. We note that the vast majority of our NALs are considerably weaker than those included in previous surveys at low spectral resolution. For example, the survey of Weymann 


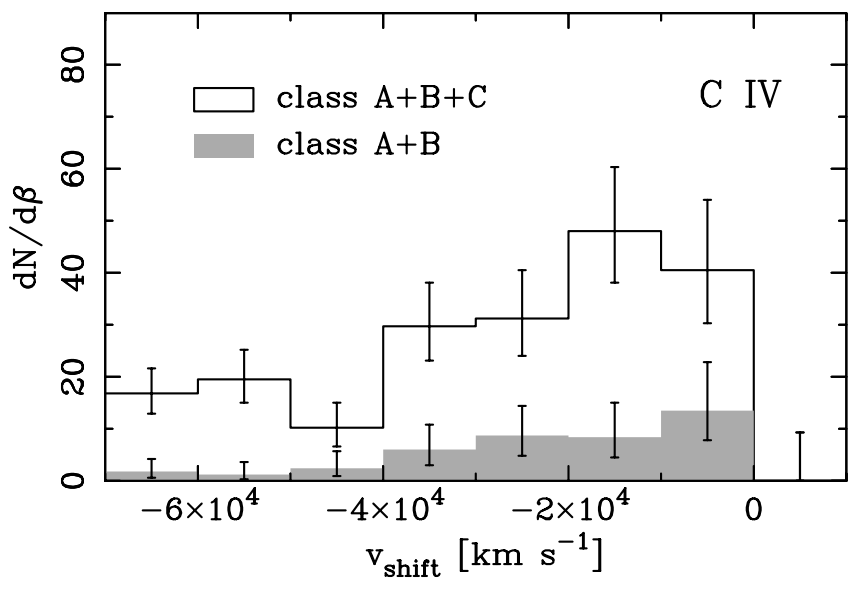

FIG. 5.-Velocity offset distribution of all $\mathrm{C}$ IV systems compared to the distribution of class $\mathrm{A}+\mathrm{B}$ (reliably and possibly intrinsic) $\mathrm{C}$ IV systems.

et al. (1979) has a rest-frame equivalent width limit of about $0.6 \AA$ (higher than $87 \%$ of the NALs in our C IV sample), while the surveys of Young et al. (1982) and F86 have a detection limit of $0.3 \AA$ (higher than $70 \%$ of the NALs in our sample). The more recent Vestergaard (2003) study was also conducted using low spectral resolution, so it focused on $\mathrm{C}$ IV absorption lines stronger than $\sim 0.3 \AA$.

A comparison of the equivalent width distributions in our study and these previous low-resolution studies is a useful indication of possible biases due to quasar properties. However, such a comparison must focus only on our stronger systems. Also, we must group together different lines that fall within $200 \mathrm{~km} \mathrm{~s}^{-1}$ of each other in order to compare with systems found at low resolution (as we did in Fig. 6). First, we focus on systems with $W_{r}(\mathrm{C}$ IV $)>1.5 \AA$ since we are concerned about possible biases due to rejecting BAL quasars from our sample, and since Vestergaard (2003) targeted the strongest systems as likely intrinsic candidates. If we take into account Vestergaard's different method of measuring the equivalent width of $\mathrm{C}$ IV, we find that her sample includes $6 / 114$ quasars that have systems with $W_{r}(\mathrm{C}$ IV $)>1.5 \AA$. However, the Vestergaard (2003) sample has approximately equal numbers of radio-loud and radio-quiet quasars, unlike ours, in which only $\frac{1}{3}$ of the quasars are radioloud. If we consider only her radio-quiet sample, only $1 / 48$ quasars has a $\mathrm{C}$ IV NAL with $W_{r}(\mathrm{C}$ IV $)>1.5 \AA$. In her radio-loud

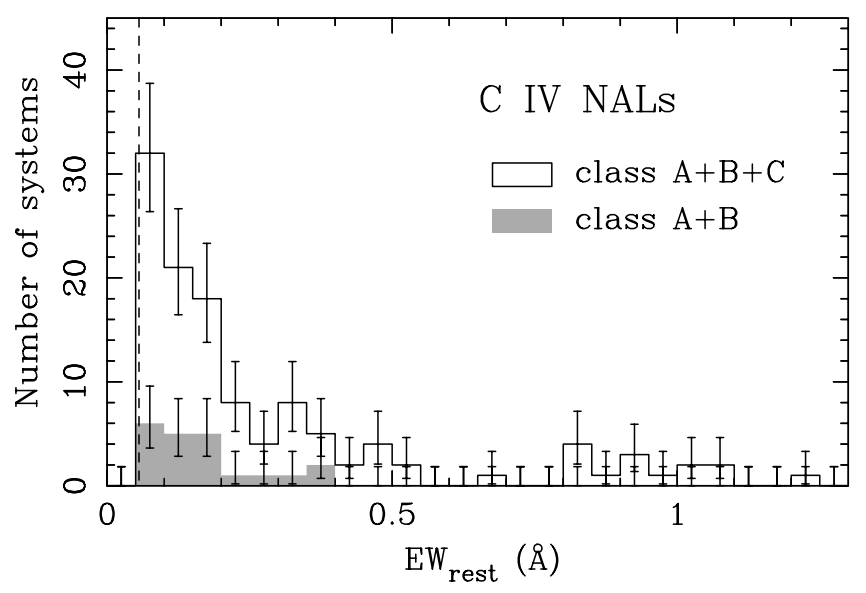

FIG. 6.-Distribution of rest-frame equivalent width of all C IV systems, compared to the distribution of class $\mathrm{A}+\mathrm{B}$ (reliably and possibly intrinsic) $\mathrm{C}$ IV NALs. The vertical dashed line marks our detection limit of $0.056 \AA$.

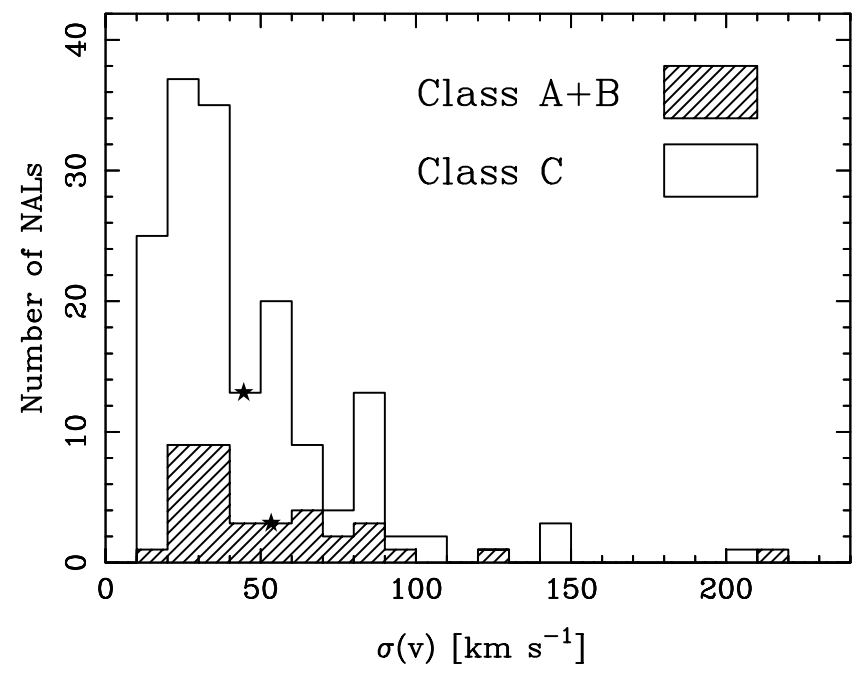

FIG. 7.-Distribution of the flux-weighted line width $[\sigma(v)$; calculated by eq. (4)] of intrinsic NALs (class A and B) and intervening/unclassified NALs (class C). The filled stars indicate the average $\sigma(v)$ values for intrinsic NALs $\left(53.4 \mathrm{~km} \mathrm{~s}^{-1}\right)$ and intervening NALs $\left(44.5 \mathrm{~km} \mathrm{~s}^{-1}\right)$.

sample, 5/66 quasars have very strong C IV NALs. Thus, we would predict that in our sample of 37 quasars (with only 12 radio-loud) we would find 1.4 very strong C IV NALs. Although we did not find any, our number is certainly consistent with this estimate, and we do find a system with $W_{r}(\mathrm{C}$ IV $) \sim 1.0 \AA$, just below that limit. We note that due to the nature of Vestergaard's study, her sample has a larger than representative fraction of radio-loud quasars. Our sample also has a somewhat larger fraction of radio-loud quasars $(37 \%)$ compared to the general population $(\sim 10 \%)$, which would lead to a bias toward detecting very strong NALs compared to the general population.

We next consider somewhat weaker NALs, down to $W_{r}(\mathrm{C}$ IV $)=$ $0.3 \AA$, to facilitate another comparison with the work of Vestergaard (2003). She found that $27 \%$ of the quasars in her sample had C IV absorption for which the sum of the equivalent widths of the two members of the doublet was $>0.5 \AA$. In our sample, we only have 13 quasars for which most of the $C$ IV associated region is covered. Of those 13 quasars, 9 have $W_{r}(\mathrm{C}$ IV $)>$ $0.056 \AA$ NALs, but only $1(8 \%)$ has $W_{r}(\mathrm{C}$ IV $)>0.3 \AA$. This is somewhat smaller than we would predict $(3.5 / 13=27 \%)$ based on Vestergaard's results; however, we again note the differences in the radio properties between the samples. Furthermore, we do not have full coverage of the associated region in all of the 13 quasars, and only 3 of the 13 are radio-loud. It is also worth noting that typically the Vestergaard (2003) quasars are 1-2 mag less luminous than the quasars in our sample.

\subsection{Line Width Distribution}

Historically, it has been very difficult to separate intrinsic NALs from intervening NALs without high-resolution spectra because of the similarities of their line profiles. However, if intrinsic NALs arise from absorbers similar to those producing BAL and mini-BAL systems (widths $\gtrsim 2000 \mathrm{~km} \mathrm{~s}^{-1}$ ), they could have larger widths compared to those of intervening NALs. If a tendency for large widths is found among intrinsic NALs, this may be used as an additional indicator of their nature. In Figure 7 we compare the distribution of the flux-weighted line width $[\sigma(v)$; see eq. (7)] of intrinsic NALs (classes A and B) to that of intervening/unclassified NALs (class C). We do not see any substantial differences between the distributions that would allow us to distinguish between intervening and intrinsic NALs. 
The average value of $\sigma(v)$ for intrinsic NALs $\left(53.4 \mathrm{~km} \mathrm{~s}^{-1}\right)$ is slightly larger than that for intervening NALs $\left(44.5 \mathrm{~km} \mathrm{~s}^{-1}\right)$. A Kolmogorov-Smirnov test gives a probability of $12 \%$ that the two distributions have been drawn by chance from the same parent population.

\subsection{Relative Numbers of Intrinsic NALs in Different Transitions}

The numbers of $\mathrm{C}$ IV, $\mathrm{N}$ v, and Si IV NALs in each of the coverage fraction classes are listed in Table $3 .{ }^{9}$ We also list in Table 3 the values of $d N / d z$ and $d N / d \beta$ for each type of system. Based on these values, reliable (class A) intrinsic NALs make up $11 \%$ of all C IV NALs, $75 \%$ of all N v NALs, and $14 \%$ of all $\mathrm{Si}$ IV NALs. These fractions increase to $19 \%, 75 \%$, and $18 \%$ after adding possible intrinsic NALs (class B). Concentrating on different transitions in turn, we note the following:

1. $\mathrm{N} v$.- The above fractions of $\mathrm{N} v \mathrm{NALs}$ that are intrinsic refer only to the AAL velocity range $\left(v_{\text {shift }} \leq-5000 \mathrm{~km} \mathrm{~s}^{-1}\right)$ because contamination by the Ly $\alpha$ forest prevented us from searching for NALs at larger velocity offsets. Nonetheless, it is worth noting that $\frac{3}{4}$ of these associated $\mathrm{N} v$ NALs are intrinsic. The density of intrinsic NALs in the associated region is thus quite high $(d N / d z=3.4$ and $d N / d \beta=13.7)$.

2. C IV.- The fraction of intrinsic $\mathrm{C}$ IV NALs in associated regions $(33 \%)$ is slightly higher than the fraction we found in nonassociated regions $(10 \%-17 \%)$. If the fraction of intrinsic NALs at $v_{\text {shift }}>-5000 \mathrm{~km} \mathrm{~s}^{-1}$ was actually the same as the fraction at $v_{\text {shift }}<-5000 \mathrm{~km} \mathrm{~s}^{-1}$, the probability that three or more out of nine $\mathrm{C}$ IV AALs are intrinsic to the quasar (based on the binomial distribution) is $5.1 \%$. If we consider only the radio-quiet subsample, the probability is even smaller, $1.2 \%$. In the radio-loud sample, we found no intrinsic $\mathrm{C}$ IV AALs, but this does not mean that there is an actual deficiency of intrinsic $\mathrm{C}$ IV AALs because our 12 spectra of radio-loud quasars have only a small redshift path for detecting C IV AALs $(\delta z=0.64)$.

3. Si IV.-We found no intrinsic Si IV AALs in the redshift window of $\delta z=1.26$ that we searched.

\subsection{Fraction of Quasars Showing Intrinsic NALs}

To investigate the geometry of absorbing gas around the quasars, we count the number of intrinsic NALs per quasar, and we evaluate the fraction of quasars hosting intrinsic NALs. These values constrain the global covering factor ${ }^{10}(\Omega / 4 \pi)$ of the continuum source and BELR by the absorber and the distribution of absorbing gas around the quasar central engine. At $z<1$, G01 found that radio-loud, flat-spectrum quasars with compact radio morphologies (i.e., with a face-on accretion disk) lack AALs down to a limiting equivalent width of $W_{\text {rest }}=0.34 \AA$. This suggests that the presence of intrinsic NALs depends on the inclination of the line of sight to the symmetry axis of the quasar central engine.

In Table 3 we list the fraction of NALs that are intrinsic and the fraction of quasars that have intrinsic NALs. We count only quasars that have velocity windows for NAL detection of $\delta \beta>$

\footnotetext{
9 We considered also subsamples of radio-loud and radio-quiet quasars but found no significant differences from the total sample.

${ }^{10}$ Here it is important to make the distinction between the terms "coverage fraction" and "global covering factor." The former term (defined in $\S 3.1$ ) refers to the fraction of the projected area of the background source occulted by an absorbing parcel of gas or, equivalently, to the fraction of background photons that pass through the absorber. The latter term refers to the total solid angle subtended by the ensemble of absorbers around the source.
}

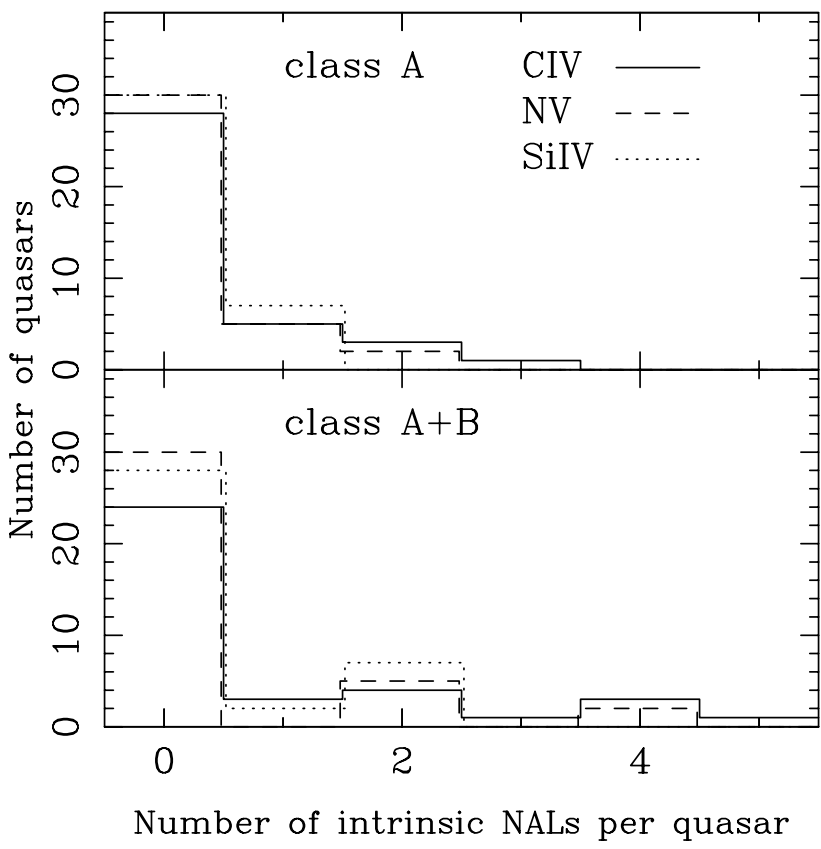

FIG. 8.-Distribution of the number of intrinsic systems per quasar. The top panel includes only class A (reliable) systems, while the bottom panel includes class $\mathrm{A}+\mathrm{B}$ (reliable+possible) systems. In each panel we show the distribution of $\mathrm{C}$ IV systems as a solid line, the distribution of $\mathrm{N} v$ systems as a dashed line, and the distribution of Si IV systems as a dotted line. We note that the number of intrinsic systems per quasar in these histograms is strictly a lower limit (see discussion in $\S 4$ ).

$0.01\left(\delta v>3000 \mathrm{~km} \mathrm{~s}^{-1}\right)$ in the transition of interest. In Figure 8 we plot the distribution of the number of intrinsic NALs per quasar. The top panel shows the distribution of only class A NALs, while the bottom panel includes both class A and B NALs.

About $24 \%$ of quasars ( 9 out of 37 ) have at least one reliable (class A) intrinsic C IV NAL. Taking possible (class B) NALs into consideration, $32 \%$ (12 out of 37) of quasars have at least one intrinsic $\mathrm{C}$ IV NAL. The corresponding fractions for $\mathrm{N} v$ and Si Iv $(19 \%-24 \%)$ are lower than for C IV. However, we cannot immediately conclude that the global covering factor of $\mathrm{C}$ IV absorbers is higher than those of $\mathrm{N} \mathrm{V}$ and $\mathrm{Si}$ Iv because all of these values are just lower limits since some intrinsic NALs could have full coverage ${ }^{11}$ and also because we were not able to search for $\mathrm{N} v \mathrm{NALs}$ over a wide range in $v_{\text {shift }}$. If we consider all three transitions, $43 \%$ (16 out of 37) of the quasars have at least one class A intrinsic NAL (in at least one of the three transitions) and $54 \%$ (20 out of 37 ) of the quasars have at least one class A or class B intrinsic NAL.

\subsection{Distribution of Coverage Fractions}

The normalized distribution of the coverage fraction for individual NAL components is presented in Figure 9, where it is broken down by transition and by reliability class. We plot only components for which we get physical covering fractions. The mean and median values of $C_{f}$ in class A+B NALs are, respectively, 0.38 and 0.33 for $\mathrm{N} \mathrm{v}, 0.50$ and 0.48 for $\mathrm{C}$ IV, and 0.56 and 0.52 for Si Iv. Putting together NALs from all three transitions, we find mean and median values of $C_{f}$ of 0.48 and 0.45 ,

11 A parcel of gas in the vicinity of the continuum source could be large enough to cover the source completely. In such a case our partial coverage method will yield $C_{f}=1$, even though the absorption lines are really intrinsic. 

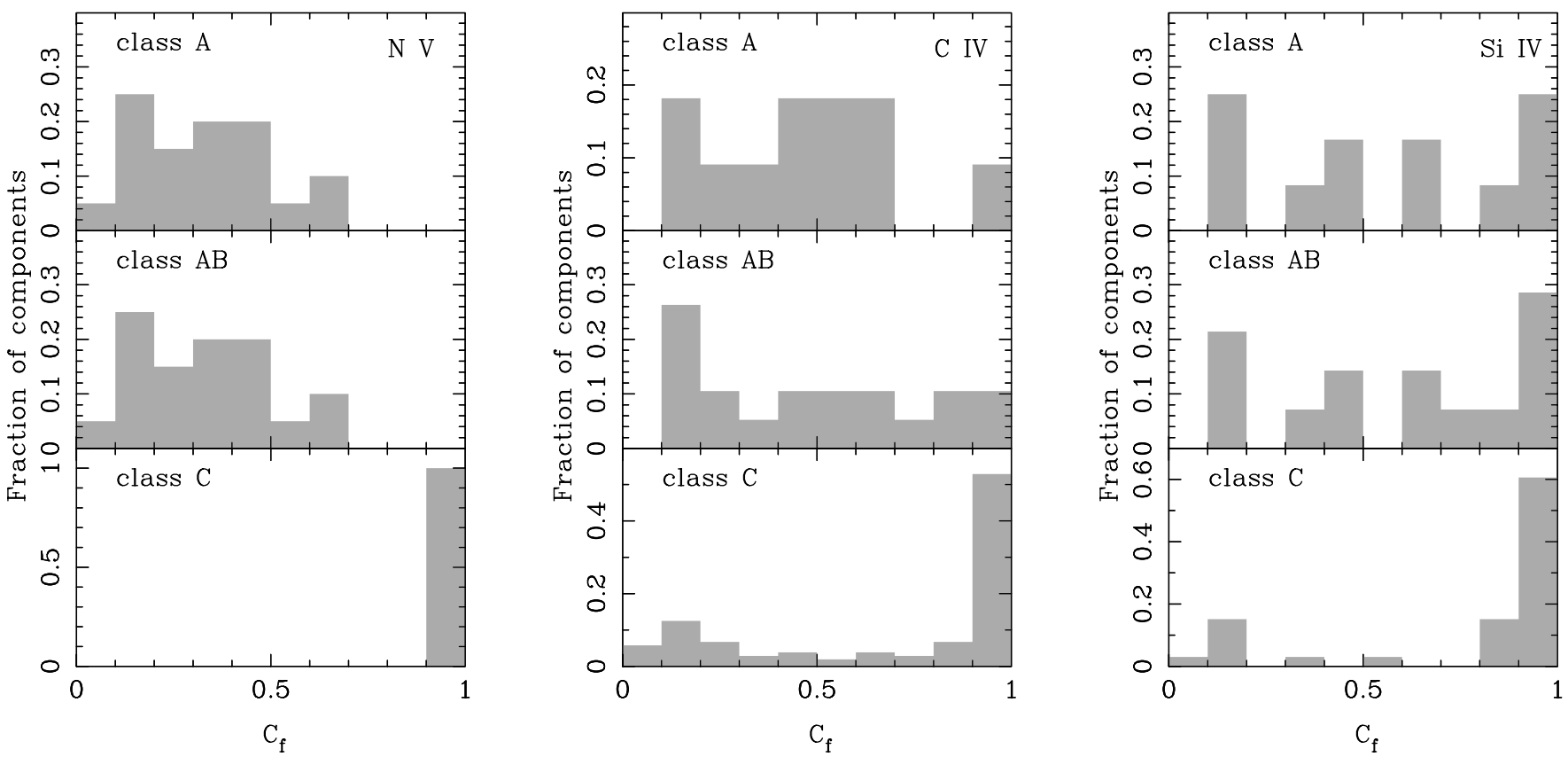

FIG. 9.-Distribution of coverage fractions of $\mathrm{C}$ Iv, $\mathrm{N}$ v and $\mathrm{Si}$ iv NAL components. We plot only components whose $C_{f}$ values are physical (i.e., $0<C_{f}$ and $C_{f}<1$ ) and evaluated with high reliability [i.e., $\sigma\left(C_{f}\right)<0.1$ ]. For each transition we plot separately the distribution of class A NALs (reliable), class A+B NALs (reliable+ possible), and class C NALs (intervening/unclassified). Even though some class C NALs have $C_{f}<1$, these values are consistent with 1 within their $1 \sigma$ errors.

respectively. Focusing on the $C_{f}$ distribution among C IV NALs (the largest of all populations shown in Fig. 9), we note that in class A+B NALs are distributed uniformly between 0 and 1, while more than half of the class C NALs have coverage fraction larger than 0.9 (this was one of the criteria for placing a component in this class).

In addition to the NAL components shown in Figure 9, Table 2 shows that $56 \%, 34 \%$, and $58 \%$ of C IV, N v, and Si Iv NAL components in the homogeneous sample have unphysical measured $C_{f}$ values. This usually indicates a doublet ratio slightly greater than 2 . If we assume that all components whose $C_{f}$ values are unphysical or are consistent with full coverage [i.e., $\left.C_{f}+\sigma\left(C_{f}\right) \geq 1.0\right]$ really have full coverage, we find that $73 \%$ (355/483), 41\% (17/41), and 72\% (131/182) of C IV, N v, and $\mathrm{Si}$ IV components cover the background flux sources. If we consider only intrinsic systems, these fractions would be $59 \%(44 / 75)$, $26 \%(8 / 31)$, and $31 \%(8 / 26)$, respectively. Generally, we display only components whose $C_{f}$ values are physical and whose errors are small enough [i.e., $\left.\sigma\left(C_{f}\right)<0.1\right]$ to avoid any uncertainties from $C_{f}$ value estimation.

For some intrinsic NAL systems, coverage fractions have been determined for more than one transition (e.g., Ganguly et al. 2003; Yuan et al. 2002). G99 found that the $C$ IV, N v, and $\mathrm{Si}$ IV coverage fractions are similar to each other for a few systems, while Petitjean \& Srianand (1999) and Srianand \& Petitjean (2000) noted that higher ionization transitions tend to have larger coverage fraction than lower ionization transitions. This type of effect could occur if the sizes of the background sources (i.e., continuum source, BELR) and/or the absorber are not the same for all transitions.

Unfortunately, our results do not allow a direct comparison between different transitions within the same system because (1) detecting multiple transitions from the same system was rather rare and (2) multiple components in the same intrinsic NAL often have different $C_{f}$ values, preventing us from assigning a single $C_{f}$ value to a NAL or a system. Therefore, we com- pare the overall distributions of the physically meaningful (between 0 and 1) $C_{f}$ values of the $34 \mathrm{C} \mathrm{IV}, 25 \mathrm{~N} \mathrm{v}$, and $20 \mathrm{Si}$ IV components found in $23 \mathrm{C}$ Iv, $9 \mathrm{~N} \mathrm{v}$, and $9 \mathrm{Si}$ IV class $\mathrm{A}$ and $\mathrm{B}$ intrinsic NALs, in Figure 9. An alternative illustration of these distributions is shown in Figure 10, where we plot the coverage fraction of each NAL component against its offset velocity. A visual inspection of these two figures suggests that the associated intrinsic $\mathrm{N} v$ NALs prefer smaller values of $C_{f}$ than the $\mathrm{C}$ IV and Si IV intrinsic NALs. Nearly all $\mathrm{N} v$ components show coverage fractions less than 0.5 , while the coverage fractions of $\mathrm{C}$ IV and Si IV NALs range from nearly 0 up to nearly 1 . This difference is also reflected in the mean and median values of $C_{f}$ reported above. However, a Kolmogorov-Smirnov test yields a probability of $17 \%$ that the $\mathrm{C}$ IV and $\mathrm{N} v C_{f}$ distributions were drawn from the same parent population. Similarly, the probability that the $\mathrm{N}$ v and $\mathrm{Si}$ IV $C_{f}$ distributions were drawn from the same parent population is $7 \%$. Larger samples are needed to reach a definitive conclusion.

\subsection{Other Relations between Intrinsic NAL and Quasar Properties}

With our large sample of intrinsic NALs we can search for relations between the NAL properties (e.g., $C_{f}$ vs. $v_{\text {shift }}$ and rest equivalent width vs. $\left.v_{\text {shift }}\right)$ and the properties of the quasars hosting them $\left(L_{\text {opt }}, L_{\text {radio }}, \mathcal{R}\right)$. Figure 11 shows plots of these parameters against each other, including class $\mathrm{A}+\mathrm{B}$ NALs. For some quasars, we plot just the upper limits on $L_{\text {radio }}$, since the corresponding radio sources have not been detected. We do not find any significant correlations in these plots. We also searched for a correlation between the offset velocity and the coverage fractions and rest equivalent width of $\mathrm{C}$ IV, $\mathrm{N}$ v, and $\mathrm{Si}$ IV NALs, but we did not find any (see Fig. 10).

\subsection{Ionization State}

Traditionally, BALs have been classified according to their ionization level into HiBALs ( high-ionization BALs), LoBALs 


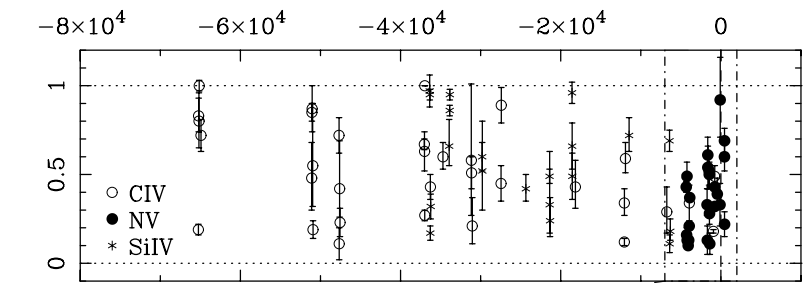

$v^{4}$
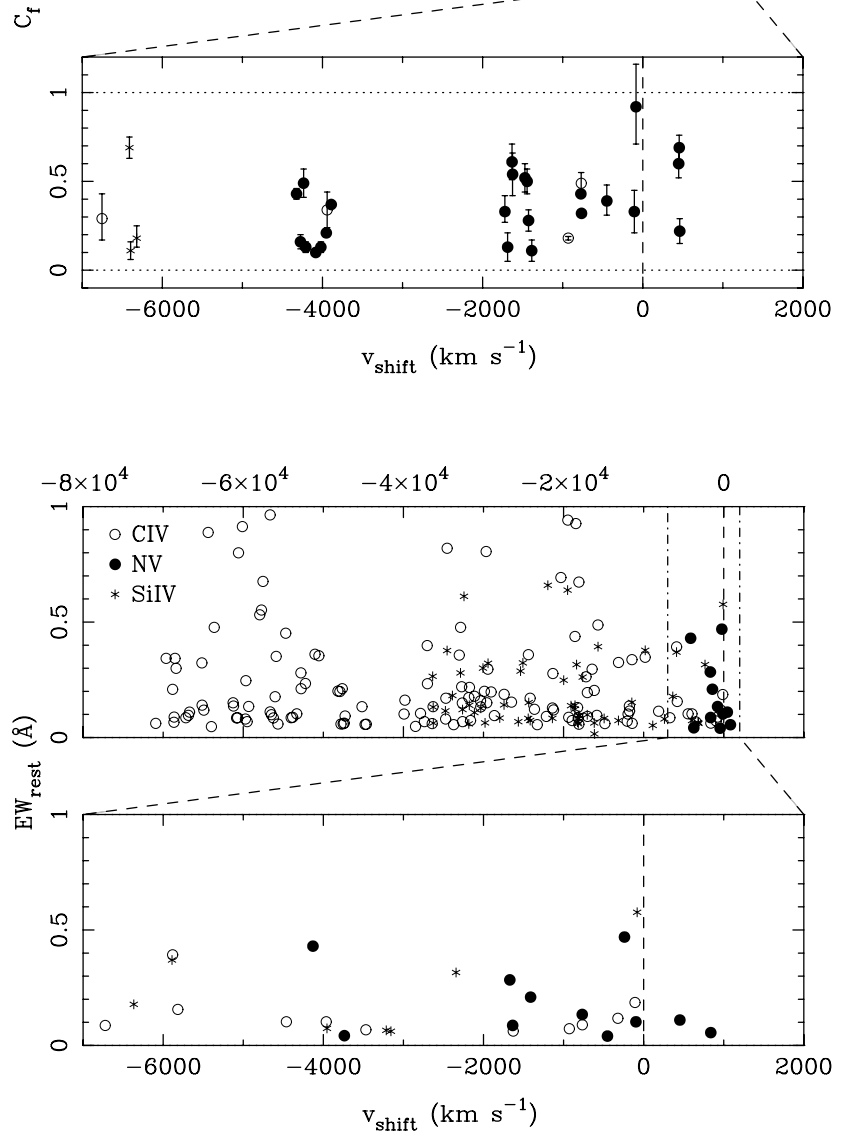

FIG. 10.-Relationship between the velocity offset of intrinsic NALs (class $\mathrm{A}+\mathrm{B}$ ) from the quasars and their coverage fraction (top) and rest-frame equivalent width (bottom). In the top panel, we plot only components whose $C_{f}$ values are physical (i.e., $0<C_{f}$ and $C_{f}<1$ ) and evaluated with high reliability [i.e., $\left.\sigma\left(C_{f}\right)<1.0\right]$. The upper panel in each case presents a wide velocity region (from $-80,000$ to $10,000 \mathrm{~km} \mathrm{~s}^{-1}$ ). The lower panel focuses on the narrower velocity window from -7000 to $2000 \mathrm{~km} \mathrm{~s}^{-1}$. Open circles denote C Iv NALs, filled circles denote N v NALs, and asterisks denote Si Iv NALs.

(low-ionization BALs), and FeLoBALs (extremely low ionization BALs that show Fe II lines; e.g., Weymann et al. 1991). Motivated by the classification of BALs and by the work of Bergeron et al. (1994) on classifying intervening NALs, we attempt to classify intrinsic NALs (classes A plus B) in a similar manner. We consider the range of conditions in the systems presented in this paper in conjunction with our concurrent study of lower redshift intrinsic NALs in the Hubble Space Telescope Space Telescope Imaging Spectrograph (STIS) Echelle archive (R. Ganguly et al. 2007, in preparation). This low-redshift sample adds an important element because the $\mathrm{O}$ vi doublet can often be examined, unlike in the spectra of $z=2-4$ quasars of this paper for which the $\operatorname{Ly} \alpha$ forest is quite thick.

We find that we can classify the intrinsic NALs in our sample into two categories according to the relative absorption strengths in various transitions:
1. Strong C iv NALs.- - In these systems Ly $\alpha$ is often saturated and black. $\mathrm{N} v$ is detected in some of these $\mathrm{C}$ IV systems, but it is weaker than $\mathrm{C}$ IV.

2. Strong $\mathrm{N} v$ NALs. - The corresponding Ly $\alpha$ lines have an equivalent width that is less than twice the $\mathrm{N} v \lambda 1239$ equivalent width. $\mathrm{C}$ Iv is detected in some of these $\mathrm{N} v$ systems but is typically weaker than $\mathrm{N} \mathrm{v}$.

To clarify, in the case of a NAL system that has both C Iv and $\mathrm{N} v$ transitions, we classify it as a strong $\mathrm{C}$ IV system if Ly $\alpha$ in the system is black or if the total equivalent width of Ly $\alpha$ is larger than twice the $\mathrm{N} v$ equivalent width. Otherwise, we classify them as a strong $\mathrm{N} v$ system, even if $\mathrm{C}$ IV is stronger than $\mathrm{N}$ v.

One possible explanation for the physical difference between $\mathrm{C}$ IV and $\mathrm{N} v$ systems is a difference in ionization parameter; however, the column density, the metallicity, and/or the metal abundance pattern could also affect these conditions. Photoionization models will be required for the conclusive discussion (J. Wu et al. 2007, in preparation).

We applied the above criteria to 28 class A and 11 class B NALs and classified them into 28 strong C IV systems (17 class A and 11 class B) and 11 strong $\mathrm{N} v$ systems (all of them are class A). The $z_{\text {abs }}=2.7164$ system toward HS $1700+1055$ has an $\mathrm{O}$ vi doublet that is much stronger than the $\mathrm{N} \mathrm{v}, \mathrm{C}$ IV, and Ly $\alpha$ lines. Although we classified it as a $\mathrm{N} v$ intrinsic NAL, it would likely fall in the additional category of a strong $\mathrm{O}$ vI system, if we allowed for that category. Finally, we added a subclassification based on the detection of low-ionization (IP $<$ $25 \mathrm{eV}$ ) or intermediate-ionization ( $\mathrm{IP}=35-50 \mathrm{eV})$ absorption lines in the same system. We note that BAL systems sometimes have extremely low ionization transitions such as $\mathrm{Mg}$ II (IP = $15 \mathrm{eV}$; e.g., Hall et al. 2002). The results are summarized in Table 4, where we report the transitions detected in each of the above systems. Notes on individual systems and velocity plots are included in the Appendix.

Table 4 shows that among the $28 \mathrm{C}$ IV intrinsic systems, 15 have low-ionization lines, while 25 have intermediate-ionization lines. Two systems (at $z_{\mathrm{abs}}=2.7699$ in Q1425+6039 and at $z_{\mathrm{abs}}=2.3198$ in Q1548+0917) are sub-DLA systems, which casts some doubt on their intrinsic classification (see the Appendix for a discussion of these individual cases). Nonetheless, we see that more than half of the intrinsic $\mathrm{C}$ iv NALs have related low-ionization absorption lines. Among the $11 \mathrm{~N}$ v systems, 5 have intermediateionization absorption, and only 1 has low-ionization absorption. This is another noteworthy difference between $\mathrm{C}$ IV and $\mathrm{N} v$ intrinsic NALs.

In $41 \%$ of intrinsic systems (16 out of 39 ) we detect lowionization transitions, and 55\% of quasars (11 out of 20) that have at least one intrinsic system have at least one system with low-ionization transitions. This is much higher than the fraction of LoBAL quasars among BAL quasars $(17 \%$ according to Sprayberry \& Foltz 1992; 13\% according to Reichard et al. 2003).

Unfortunately, $\mathrm{O}$ VI is in the $\mathrm{Ly} \alpha$ forest or is not covered in our spectra, making it quite difficult to establish the ionization properties of our systems. However, an additional class of $\mathrm{O}$ VI associated NALs (possibly intrinsic) has emerged from our study of lower redshift quasars using the STIS Echelle archive. These $\mathrm{O}$ vi systems are sometimes redward of the emission redshift of the quasar, they have relatively weak Ly $\alpha$ lines compared to $\mathrm{O} \mathrm{VI}$, and they do not always have partial coverage (R. Ganguly et al. 2007, in preparation). A few of our N v 


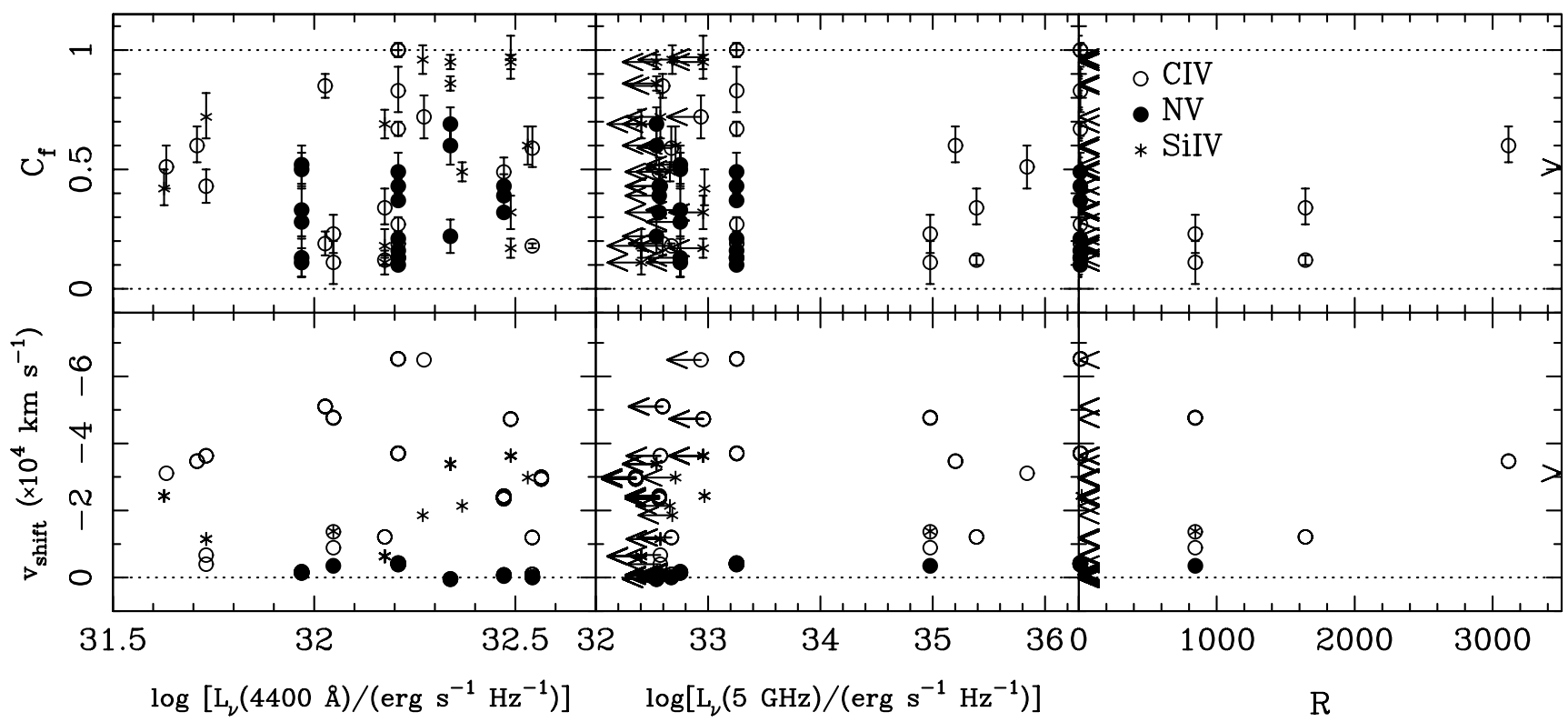

FIG. 11.-Plots of NAL parameters (i.e., $C_{f}$ and $v_{\text {shift }}$ ) against quasar properties [i.e., $L_{\nu}(4400 \AA), L_{\nu}(5 \mathrm{GHz})$, and $\mathcal{R}$ ]. Only intrinsic NAL components (class A+B), whose $C_{f}$ values are physical (i.e., $0<C_{f}$ and $C_{f}<1$ ) and evaluated with high reliability [i.e., $\sigma\left(C_{f}\right)<0.1$ ], are plotted. For quasars that were not detected in the radio, we plot upper limits on $L_{\nu}(5 \mathrm{GHz})$ and $\mathcal{R}$. Open circles denote $\mathrm{C}$ iv NALs, filled circles denote N v NALs, and asterisks denote Si Iv NALs.

intrinsic NALs with nonblack Ly $\alpha$ may have very strong O vI absorption and thus could fall in this third category.

\section{DISCUSSION}

\subsection{Evolution of the Frequency of Intrinsic NALs with Redshift}

If we associate intrinsic NALs with quasar outflows, their evolution with redshift traces the evolution of quasar outflows. Moreover, since outflow properties depend on the properties of the accretion flow (at least in disk wind models), the redshift evolution of NALs provides constraints on the evolution of quasar fueling rates.

We begin by considering quasars with strong $\mathrm{C}$ IV AALs ( $W_{\text {rest }} \geq 1.5 \AA$ ), which have a high probability of being intrinsic because of their small velocity offsets from the quasars. At intermediate redshifts $(z=1-2)$, F86 found strong $C$ IV AALs in $33 \%$ of quasars, while G01 detected such a strong C IV AAL in only $2 \%$ of low-redshift quasars $(z<1)$ and suggested that this fraction increases with redshift. In our high-redshift sample $(z=2-4)$, we only detect one rather strong AAL with $W_{\text {rest }} \sim$ $1.0 \AA$. This could mean that the frequency of strong AALs peaks at $z \sim 2$, which coincides with the redshift at which the quasar density peaks. There are, however, a number of noteworthy caveats to this apparent evolutionary trend, namely, (1) the samples used at different redshifts have different optical and radio luminosity distributions (the $\mathrm{G} 01$ sample includes a significant fraction of low-luminosity objects, for example); (2) strong AALs appear to prefer steep-spectrum radio-loud quasars (F86; Anderson et al. 1987), which make up different fractions of the above samples (the F86 sample is predominantly radio-loud); and (3) strong AALs need not always be intrinsic, in particular, they could come from galaxies in a cluster surrounding the quasar, which are more often found around radio-loud quasars (e.g., Yee \& Green 1987; Ellison et al. 1991; Yee \& Ellison 1993; Wold et al. 2000).

We now turn our attention to the evolution of the fraction of quasars with intrinsic AALs, including weak ones, down to a rest-frame equivalent width of $\sim 50 \mathrm{~m} \AA$ (using samples constructed on the basis of partial coverage or variability). At $z<1.5$, Wise et al. (2004) found that a minimum of $27 \%$ of quasars have intrinsic AALs, while at $z \sim 2$, Narayanan et al. (2004) found that a minimum of $25 \%$ of quasars host intrinsic AALs. Similarly, G99 find that at least $50 \%$ of $z \sim 2$ quasars have intrinsic AALs, and in this study we find that a minimum of $23 \%$ of quasars at $z=2-4$ have intrinsic AALs. These fractions are consistent with each other, especially considering that some of the samples are rather small (only six quasars in the G99 sample). An important caveat here is that all of these results refer to lower limits since NALs that do not show variability or partial coverage can still be intrinsic. This caveat not withstanding, the fraction of quasars hosting intrinsic AALs does not show significant redshift evolution. A closely related question is the redshift evolution of the fraction of AALs that are intrinsic. At $z<1.5$ this fraction is $>21 \%$ (Wise et al. 2004), at $z \sim 2$ it is $>23 \%$ (Narayanan et al. 2004) or $>60 \%$ (G99), and at $z=2-4$ it is $>27 \%$ (this work). Thus, this quantity does not appear to evolve with redshift either, but this conclusion is also subject to the caveat noted above.

\subsection{Properties of Intrinsic Absorbers in Our Sample}

\subsubsection{Range of Coverage Fractions}

In nonassociated regions (i.e., $\left|v_{\text {shift }}\right|>5000 \mathrm{~km} \mathrm{~s}^{-1}$ ), we found that $10 \%-17 \%$ of C IV NALs are intrinsic to the quasars. Richards et al. (1999) and Richards (2001) suggested that this fraction is as large as $36 \%$, based on the excess of absorbers at $>5000 \mathrm{~km} \mathrm{~s}^{-1}$ in steep spectrum as compared to flat-spectrum radio-loud quasars. Our estimate is strictly only a lower limit because not all intrinsic NALs can be identified using partial coverage. If we take both our result that $10 \%-17 \%$ of C IV NALs are intrinsic and the Richards et al. (1999) number of $36 \%$ at face value and try to reconcile them, we conclude that only $30 \%-$ $50 \%$ of intrinsic C IV NALs exhibit detectable partial coverage in our sample. We caution that the two samples were selected in different ways, leading to biases. For example, the sample of Richards et al. (1999) has a larger fraction $(\sim 50 \%)$ of radioloud quasars than our sample (32\%). However, since they found that intrinsic NALs prefer radio-quiet quasars, the difference 
TABLE 4

Ionization Levels of Class A and Class B Systems

\begin{tabular}{|c|c|c|c|c|c|c|c|}
\hline $\begin{array}{l}\text { QSO } \\
(1)\end{array}$ & $\begin{array}{l}z_{\text {abs }} \\
(2)\end{array}$ & $\begin{array}{c}\text { Low-Ion. }^{\text {a }} \\
13-24 \mathrm{eV} \\
\text { (3) }\end{array}$ & $\begin{array}{c}\text { Interm.-Ion. }^{\text {b }} \\
33-48 \mathrm{eV} \\
\text { (4) }\end{array}$ & $\begin{array}{c}\text { C IV } \\
65 \mathrm{eV} \\
(5)\end{array}$ & $\begin{array}{c}\mathrm{N} \mathrm{v} \\
98 \mathrm{eV} \\
(6)\end{array}$ & $\begin{array}{c}\mathrm{O} \mathrm{vI} \\
138 \mathrm{eV} \\
(7)\end{array}$ & $\begin{array}{c}\text { Ionization } \text { Class }^{\mathrm{c}} \\
\text { (8) }\end{array}$ \\
\hline \multicolumn{8}{|c|}{ Class A Systems } \\
\hline \multirow[t]{2}{*}{ 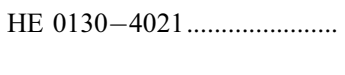 } & 2.5597 & $\mathrm{~N}$ & $\mathrm{Y}$ & $\mathrm{Y}$ & $\mathrm{N}$ & $\ldots$ & 1, HI \\
\hline & 2.9749 & $\mathrm{~N}$ & $\mathrm{~N}$ & $\ldots$ & $\mathrm{Y}$ & $\mathrm{Y}$ & $2, \mathrm{H}$ \\
\hline \multirow{2}{*}{ HE $0322-3213 \ldots \ldots \ldots \ldots \ldots \ldots \ldots$} & 3.2781 & $\mathrm{~N}$ & $\mathrm{~N}$ & $\ldots$ & $\mathrm{Y}$ & $\ldots$ & $2, \mathrm{H}$ \\
\hline & 3.2818 & $\ldots$ & $\mathrm{Y}$ & $\ldots$ & $\mathrm{Y}$ & $\ldots$ & 2, HI \\
\hline Q0450-1310.............................. & 2.2307 & $\mathrm{~N}$ & $\mathrm{Y}$ & $\ldots$ & $\mathrm{Y}$ & $\ldots$ & 2, HI \\
\hline Q0636+6801 .......................... & 3.0134 & $\mathrm{~N}$ & $\mathrm{Y}$ & $\mathrm{Y}$ & $\ldots$ & $\ldots$ & 1, HI \\
\hline Q0642+4454 .......................... & 2.9721 & $\mathrm{~N}$ & $\mathrm{Y}$ & $\mathrm{Y}$ & $\ldots$ & $\ldots$ & 1, HI \\
\hline \multirow[t]{3}{*}{ 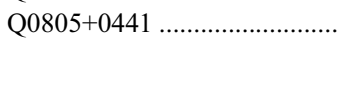 } & 2.4544 & $\mathrm{~N}$ & $\mathrm{Y}$ & $\mathrm{Y}$ & $\mathrm{N}$ & $\ldots$ & $1, \mathrm{HI}$ \\
\hline & 2.6517 & $\mathrm{Y}$ & $\mathrm{Y}$ & $\mathrm{Y}$ & $\mathrm{Y}$ & $\ldots$ & 1, HIL \\
\hline & 2.8589 & $\mathrm{~N}$ & $\mathrm{~N}$ & $\mathrm{Y}$ & $\mathrm{Y}$ & $\mathrm{Y}$ & $2, \mathrm{H}$ \\
\hline \multirow[t]{2}{*}{ Q1009+2956 } & 2.2533 & $\mathrm{Y}$ & $\mathrm{Y}$ & $\ldots$ & $\mathrm{N}$ & $\ldots$ & $1, \mathrm{HIL}$ \\
\hline & 2.6495 & $\mathrm{~N}$ & $\mathrm{Y}$ & $\ldots$ & $\mathrm{Y}$ & $\mathrm{Y}$ & 2, HI \\
\hline \multirow[t]{3}{*}{ Q1017+1055 } & $\approx 2.97$ & $\mathrm{Y}^{\mathrm{d}}$ & $\mathrm{Y}^{\mathrm{d}}$ & $\mathrm{Y}^{\mathrm{d}}$ & $\ldots$ & $\ldots$ & 2, HIL \\
\hline & $\approx 3.03$ & $\mathrm{~N}$ & $\mathrm{~N}$ & $\mathrm{Y}^{\mathrm{d}}$ & $\ldots$ & $\ldots$ & $2, \mathrm{H}$ \\
\hline & $\approx 3.11$ & $\mathrm{~N}$ & $\mathrm{Y}$ & $\ldots$ & $\mathrm{Y}^{\mathrm{d}}$ & $\cdots$ & $2, \mathrm{HI}$ \\
\hline 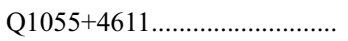 & 3.5314 & $\mathrm{Y}$ & $\mathrm{Y}$ & $\ldots$ & $\ldots$ & $\ldots$ & 1, HIL \\
\hline Q1107+4847 & 2.7243 & $\mathrm{Y}$ & $\mathrm{Y}$ & $\mathrm{Y}$ & $\mathrm{N}$ & $\ldots$ & $1, \mathrm{HIL}$ \\
\hline 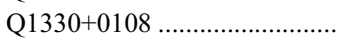 & 3.1148 & $\mathrm{Y}$ & $\mathrm{Y}$ & $\mathrm{Y}$ & $\ldots$ & $\ldots$ & 1, HIL \\
\hline 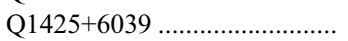 & 2.7699 & $\mathrm{Y}$ & $\mathrm{Y}$ & $\mathrm{Y}$ & $\ldots$ & $\ldots$ & 1, HIL (DLA) \\
\hline \multirow{3}{*}{ 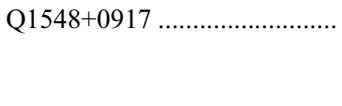 } & 2.3198 & $\mathrm{Y}$ & $\mathrm{Y}$ & $\mathrm{Y}$ & $\mathrm{N}$ & $\ldots$ & 1, HIL (DLA) \\
\hline & 2.6659 & $\mathrm{Y}$ & $\mathrm{Y}$ & $\mathrm{Y}$ & $\mathrm{N}$ & $\ldots$ & $1, \mathrm{HIL}$ \\
\hline & 2.6998 & $\mathrm{Y}$ & $\mathrm{Y}$ & $\mathrm{Y}$ & $\mathrm{N}$ & $\mathrm{N}$ & $1, \mathrm{HIL}$ \\
\hline 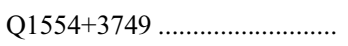 & 2.3777 & $\mathrm{Y}$ & $\mathrm{Y}$ & $\ldots$ & $\mathrm{N}$ & $\ldots$ & $1, \mathrm{HIL}$ \\
\hline \multirow[t]{2}{*}{ HS $1700+6416 \ldots \ldots \ldots \ldots \ldots \ldots \ldots \ldots$} & 2.7125 & $\mathrm{~N}$ & $\mathrm{~N}$ & $\mathrm{Y}$ & Y & $\ldots$ & $2, \mathrm{H}$ \\
\hline & 2.7164 & $\mathrm{~N}$ & $\mathrm{~N}$ & $\mathrm{~N}$ & $\mathrm{Y}$ & $\mathrm{Y}$ & $2, \mathrm{H}$ \\
\hline \multirow[t]{3}{*}{ 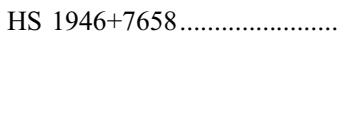 } & 2.8928 & $\mathrm{Y}$ & $\mathrm{Y}$ & $\mathrm{Y}$ & $\mathrm{N}$ & $\ldots$ & 1, HIL \\
\hline & 3.0385 & $\mathrm{~N}$ & $\mathrm{~N}$ & $\mathrm{Y}$ & $\mathrm{N}$ & $\ldots$ & $1, \mathrm{H}$ \\
\hline & 3.0497 & Y & Y & $\mathrm{Y}$ & $\mathrm{Y}$ & $\ldots$ & 1, HIL \\
\hline \multicolumn{8}{|c|}{ Class B Systems } \\
\hline 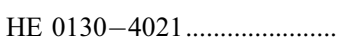 & 2.2316 & $\mathrm{~N}$ & $\mathrm{Y}$ & $\mathrm{Y}$ & $\ldots$ & $\ldots$ & 1, HI \\
\hline Q0241-0146............................... & 3.0451 & $\mathrm{~N}$ & $\mathrm{Y}$ & $\mathrm{Y}$ & $\ldots$ & $\ldots$ & 1, HI \\
\hline 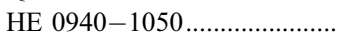 & 2.8347 & $\mathrm{~N}$ & $\mathrm{Y}$ & $\mathrm{Y}$ & $\ldots$ & $\ldots$ & 1, HI \\
\hline Q1017+1055 ............................ & 2.5408 & $\mathrm{~N}$ & $\mathrm{~N}$ & $\mathrm{Y}$ & $\mathrm{N}$ & $\ldots$ & $1, \mathrm{H}$ \\
\hline 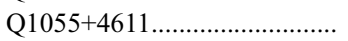 & 3.3658 & $\mathrm{~N}$ & $\mathrm{~N}$ & $\mathrm{Y}$ & $\ldots$ & $\ldots$ & $1, \mathrm{H}$ \\
\hline \multirow[t]{2}{*}{ HS $1103+6416 \ldots \ldots \ldots \ldots \ldots \ldots$} & 1.8874 & $\mathrm{~N}$ & $\mathrm{Y}$ & $\mathrm{Y}$ & $\mathrm{N}$ & $\ldots$ & $1, \mathrm{HI}$ \\
\hline & 1.8919 & $\mathrm{Y}$ & $\mathrm{Y}$ & $\mathrm{Y}$ & $\mathrm{N}$ & $\ldots$ & 1, HIL \\
\hline Q1334-0033 & 2.2010 & $\mathrm{~N}$ & $\mathrm{Y}$ & $\mathrm{Y}$ & $\mathrm{N}$ & $\ldots$ & $1, \mathrm{HI}$ \\
\hline 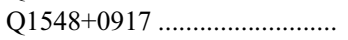 & 2.6082 & $\mathrm{Y}$ & $\mathrm{Y}$ & $\mathrm{Y}$ & $\mathrm{N}$ & $\ldots$ & $1, \mathrm{HIL}$ \\
\hline \multirow[t]{2}{*}{ HS $1700+6416 \ldots \ldots \ldots \ldots \ldots \ldots$} & 2.4330 & $\mathrm{Y}$ & $\mathrm{Y}$ & $\mathrm{Y}$ & $\mathrm{N}$ & $\ldots$ & 1, HIL \\
\hline & 2.4394 & $\mathrm{~N}$ & $\mathrm{Y}$ & $\mathrm{Y}$ & $\mathrm{N}$ & $\ldots$ & $1, \mathrm{HI}$ \\
\hline
\end{tabular}

NотE.-A " $\mathrm{Y}$ " indicates that one or more lines of a given type are detected, an " $\mathrm{N}$ " indicates that no lines from that category were detected, while ellipses mean that either the spectrum did not cover those transitions or the transitions were severely blending with Ly $\alpha$ forest or data defects.

${ }^{\text {a }}$ Low-ionization lines (from ions with ionization potentials between 13 and $24 \mathrm{eV}$ ): O I $\lambda 1302$, Si II $\lambda \lambda 1190,1193,1260,1527, \mathrm{Al}_{\text {II }} \lambda 1671, \mathrm{C}$ II $\lambda 1335$.

${ }^{b}$ Intermediate-ionization lines (from ions with ionization potentials between 33 and $48 \mathrm{eV}$ ): $\mathrm{Si}$ III $\lambda 1207, \mathrm{Si}$ IV $\lambda \lambda 1394,1403, \mathrm{C}$ III $\lambda 977$.

c $1=\mathrm{C}$ IV-dominated system, $2=\mathrm{N}$ v-dominated system. H, I, and L mean that the system contains high-, intermediate-, and low-ionization lines, respectively.

"These lines in Q1017+1055 are classified as "mini-BALs" because they have widths of 500-1000 $\mathrm{km} \mathrm{s}^{-1}$. See detailed discussion in the Appendix.

between our estimate of the fraction of quasars hosting intrinsic NALs and theirs would be larger than what it appears, leading to a larger number of fully covered intrinsic NALs.

This conclusion is bolstered by the fact that the distribution of measured coverage fractions of intrinsic C IV NALs spans the range from 0 to nearly 1 (within uncertainties). The covering factors are presumably related to the physical sizes of the absorbing structures, which could span a significant range. In this sense there is nothing special about the size corresponding to a covering factor of 1 . If there is a cutoff in size, there is no reason it would occur particularly at this value. Thus, we strongly expect that many intrinsic absorbers with unit coverage fraction exist.

\subsubsection{Ionization Conditions}

The wide range of absorber ionization conditions presented in $\S 4.7$ (e.g., "strong $\mathrm{N} \mathrm{v}$ " vs. "strong $\mathrm{C}$ Iv" absorbers) may be a result of a wide distribution of distances of the gas parcels from the continuum source and/or a wide distribution of their densities. The velocity offset of a system can serve as an indicator of 
its proximity to the continuum source under the assumption that parcels of gas in an outflowing wind are accelerated outward from the continuum source. Detailed photoionization modeling of selected systems can help us determine whether there is a relation between their ionization state of NAL systems and their apparent outflow velocity, which will allow us to determine whether there is ionization stratification in the outflow. The same models may also provide constraints on the density of the gas. In fact, photoionization models for the associated absorber in QSO J2233-606 (Gabel et al. 2006) show that the higher ionization kinematic components are at lower blueshifted velocities than lower ionization components. Moreover, preliminary results of photoionization models for three of the quasars in our sample (J. Wu et al. 2007, in preparation) suggest that absorbers at different velocities in the same quasar can have a wide range of densities. An alternative way of constraining the density is through monitoring observations, which can set limits on the recombination timescale of the absorber and hence constrain the density (see, e.g., the discussion in Wise et al. 2004; Narayanan et al. 2004; M05).

It is also important to ask whether the low-ionization transitions in intrinsic $\mathrm{C}$ IV NALs are aligned with the high-ionization transitions. An inspection of the velocity plots of individual systems in the Appendix shows that the line centers and profiles of low-ionization lines in intrinsic NAL systems are almost always similar to those of high-ionization lines. This leads us to conclude that the low-ionization lines arise in the intrinsic absorbers and not in the ISM of the host galaxy.

\subsubsection{Intrinsic NALs at Large Velocity Offsets}

Our finding (supported by the independent method of Richards 2001) that a significant fraction of NALs, even at high-velocity offsets from the quasar redshift, are intrinsic $(10 \%-17 \%$ of C IV NALs and $15 \%-20 \%$ of Si IV NALs) has implications for cosmology since it affects the estimates of the density of intervening systems per unit redshift $(d N / d z)$. For example, at $z=2-4$, $d N / d z$ is estimated to be 3.11 for $\mathrm{C}$ IV and 1.36 for $\mathrm{Si}$ IV systems with a rest-frame equivalent width greater than $0.15 \AA$ (Misawa et al. 2002). If these values are reduced by $15 \%-30 \%$ after correction for intrinsic systems, this would require some adjustment of models for cosmological evolution of these systems. Furthermore, the contamination of the $\operatorname{Ly} \alpha$ forest by very high metallicity intrinsic NALs could seriously bias estimations of the metallicity of the forest.

\subsection{Implications for Models of Quasar Outflows}

The results of our survey have direct implications for models of quasar outflows, whatever the acceleration mechanism:

1. The minimum fraction of quasars with at least one intrinsic NAL (C Iv, $\mathrm{N} v$, or $\mathrm{Si}$ IV) is $43 \%-54 \%$. If there is an additional family of intrinsic $\mathrm{O}$ VI absorbers, without corresponding $\mathrm{C}$ IV or $\mathrm{N} v$ lines, the minimum fraction of quasars with intrinsic NALs will increase further. This leads to a constraint on the solid angle subtended by the NAL absorbers to the background source(s). We can interpret this result in the context of a simple (but clearly not unique) picture in which all quasars in our sample are similar to each other and the NAL gas is located at intermediate latitudes above the streamlines of a fast, equatorial outflow (a BAL wind). This geometry is similar to that depicted in Figure 13 of G01, although it need not apply specifically to an outflow launched from the accretion disk; a pressure-driven outflow launched from a distance of an order of $1 \mathrm{pc}$ from the black hole may have a similar geometry. In this picture, the above observational constraint translates directly into the opening angle of the NAL zone, given the opening angle of the BAL wind. For an opening angle of the BAL wind of approximately $7^{\circ}-12^{\circ}$ $(10 \%-20 \%$ of quasars are BAL quasars; e.g., Hamann et al. 1993), the angular width of the NAL zone turns out to be $25^{\circ}-27^{\circ}$. In this picture the polar region, at latitudes above the NAL zone, may be filled with highly ionized gas. This idea is consistent with the finding of Barthel et al. (1997) that strong associated C IV NALs with $W_{\text {rest }}>3 \AA$ are most common at angles far from the jet axis in radio-loud QSOs (as high as $45^{\circ}$ ). Such strong NALs may represent intermediate viewing angles between intrinsic NALs and BALs. Other simple interpretations are also possible: for example, the NAL gas may be distributed isotropically around the central engine, or some quasars may be more likely than others to host such absorbers (e.g., the width of the NAL zone may depend on luminosity, as suggested by Elvis 2000).

2. The density of intrinsic (class $\mathrm{A}+\mathrm{B}$ ) $\mathrm{C}$ IV NALs per unit velocity interval, $d N / d \beta$, increases from 4.1 at $v_{\text {shift }}<$ $-5000 \mathrm{~km} \mathrm{~s}^{-1}$ to 18 at $v_{\text {shift }}>-5000 \mathrm{~km} \mathrm{~s}^{-1}$. This is a significant increase with a chance probability of only $5 \%$. These results have a number of implications for outflow models. First, the high-velocity NALs $\left(71 \%\right.$ of class A+B NALs at $v_{\text {shift }}<$ $-5000 \mathrm{~km} \mathrm{~s}^{-1}$ ) are unlikely to be associated with pressuredriven outflows because these outflows can only reach velocities of order $10^{3} \mathrm{~km} \mathrm{~s}^{-1}$. The distribution of $d N / d \beta$ with $\beta$ does not provide a straightforward constraint on the acceleration mechanism because the models do not predict how individual parcels of NAL gas are distributed. One could take the higher value of $d N / d \beta$ at low velocities as an indication of the existence of a separate population of absorbers, which could perhaps be identified with a pressure-driven wind. On the other hand, the velocity of a magnetocentrifugal or line-driven accretion disk wind increases smoothly with radius, which may lead to higher density of NALs per unit velocity at low velocities (assuming that the gas parcels are condensations that form near the base of the wind and are accelerated outward). Economy of means and Occam's razor favor the latter explanation since it applies to both high- and low-velocity NALs and invokes a single acceleration mechanism. This issue can be addressed observationally by constraining the distance of the low-velocity (associated) NAL gas from the central engine. This can be done by combining constraints on its ionization parameter (obtained from photoionization models) with constraints on its density (obtained from variability studies).

3. The $\mathrm{C}$ IV column densities derived from fitting the line profiles can yield a preliminary estimate of the total hydrogen column densities ( $\mathrm{H} \mathrm{I}+\mathrm{H}$ II) in the absorbers. More specifically, we can apply the calculations of Hamann (1997) to estimate an upper limit to the hydrogen column density of absorbers in our sample from our highest measured $\mathrm{C}$ IV column density of $\log N_{\mathrm{C} \text { IV }}=15.86$ (in the $z_{\mathrm{abs}}=2.5597 \mathrm{NAL}$ in HE 01304021). If we assume an abundance of $\mathrm{C}$ IV relative to $\mathrm{C}$ of $\log \left(N_{\mathrm{C} \text { IV }} / N_{\mathrm{C}}\right) \approx-0.34$ (this limit corresponds to the optimal ionization parameter that maximizes the $\mathrm{C}$ IV abundance; see Fig. 2 of Hamann 1997) and a solar abundance of $\mathrm{C}$ relative to $\mathrm{H}, \log (\mathrm{C} / \mathrm{H})=-3.44$ (Grevesse \& Anders 1989), we obtain $N_{\mathrm{H}} \sim 4 \times 10^{19} \mathrm{~cm}^{-2}$. If the $\mathrm{C}$ abundance is supersolar (e.g., Hamann 1997), this limit will become smaller, of course. Higher total column densities have also been observed in NALs; for example, Arav et al. (2001a) find $\log N_{\mathrm{H}}>20.3$ in QSO J23591241. Moreover, total column densities in BALs can be an order of magnitude higher (e.g., in PG 0946+301; Arav et al. 2001b).

We emphasize that the column densities estimated above refer only to the gas responsible for the C IV NALs. This gas could 
well have the form of filaments embedded in a hotter medium, as predicted by many models (see $\S 1$ and references therein). If that is the case, then the total column density of the outflow can be considerably larger than what we have estimated above. In fact, $\mathrm{X}$-ray spectroscopy of nearby AGNs does indicate a multiphase absorber structure in which the column density of the hot medium is higher than that of the cold medium by a factor of 30 or more (e.g., Netzer et al. 2003; Kaspi et al. 2004 and references therein).

For the specific case of radiatively accelerated outflows, we can ask whether the observed high velocities of some NALs are attainable in the context of the model. Hamann (1998) derives the following expression for the terminal velocity of a radiatively accelerated wind:

$$
v_{\infty}=9300 r_{0.1}^{-1 / 2}\left(\frac{f_{0.1} L_{46}}{N_{22}}-0.1 M_{8}\right)^{1 / 2} \mathrm{~km} \mathrm{~s}^{-1},
$$

where $r=0.1 r_{0.1} \mathrm{pc}$ is the distance of the absorber from the continuum source, $L=10^{46} L_{46} \mathrm{ergs} \mathrm{s}^{-1}$ is the quasar luminosity, $M_{\mathrm{BH}}=10^{8} M_{8} M_{\odot}$ is the black hole mass, $f=0.1 f_{0.1}$ is the fraction of continuum photons absorbed or scattered by the gas, and $N_{\mathrm{H}}=10^{22} N_{22} \mathrm{~cm}^{-2}$ is the column density of the absorbing gas. Noting that $L_{46} / M_{8} \approx 0.77\left(L / L_{\mathrm{Edd}}\right.$ ) (where $L_{\mathrm{Edd}}$ is the Eddington luminosity) and that $r_{0.1} / M_{8} \approx 2 \xi_{4}\left(\xi_{4} \equiv r / 10^{4} r_{g}\right.$ and $r_{g} \equiv G M_{\mathrm{BH}} / c^{2}$ is the gravitational radius), we may recast equation (13) as

$$
v_{\infty}=5000 \xi_{4}^{-1 / 2}\left(\frac{f_{0.1}}{N_{22}} \frac{L}{L_{\text {Edd }}}-0.13\right)^{1 / 2} \mathrm{~km} \mathrm{~s}^{-1} .
$$

As Hamann (1998) argues, $f_{0.1} \sim 1$ for BAL quasars. The radiation pressure-dominated part of the accretion disk extends up to $\xi_{4} \approx 0.25$ for parameters scaled as above (see Shakura \& Sunyaev 1973). According to most models for accretion disk winds (Murray et al. 1995; Proga et al. 2000; Everett 2005), the inner launch radius of the wind corresponds to $\xi_{4} \approx 0.06-0.25$. Moreover, the $\mathrm{C}$ iv column densities that we find observationally (see discussion above) indicate total hydrogen column densities in NALs corresponding to $N_{22} \sim 0.01$. Under these conditions, radiation pressure can accelerate the NAL gas to terminal speeds well in excess of $10^{5} \mathrm{~km} \mathrm{~s}^{-1}$, and the observed blueshifts of intrinsic NALs can be easily explained.

There is an important caveat to the above estimate. We have assumed that the column density that we have measured from the $\mathrm{C}$ IV NAL profiles traces all of the matter that is being accelerated. But, as we note earlier in this section, the NAL gas may be embedded in a hot medium and may represent only a small fraction of the total gas mass. In such a case, the attainable terminal velocity will decrease accordingly. More specifically, if the NAL gas represents up to $10 \%$ of the total column density, then equation (14) can still reproduce the observed NAL velocities. But if the total column density is more than an order of magnitude higher than the C IV NAL column density, then a different acceleration mechanism must be sought.

\section{SUMMARY AND CONCLUSIONS}

We have constructed a large, relatively unbiased, equivalent width-limited sample of intrinsic NAL systems found in the spectra of $z=2-4$ quasars. This sample comprises $124 \mathrm{C}$ IV, $12 \mathrm{~N} \mathrm{v}$, and $50 \mathrm{Si}$ Iv doublets, which were separated from intervening NALs on the basis of their partial coverage signature.
After assessing the reliability of the determination of the intrinsic nature of these NAL systems, 28 are deemed reliably intrinsic ("class A"), 11 are deemed possibly intrinsic ("class B"), and 111 are deemed intervening (or unreliable intrinsic candidates; "class C"). Using this sample of NAL systems, we study their demographics, the distribution of their physical properties, and any relations between them. We also consider the implications of these results for models of outflowing winds. Our findings and conclusions are as follows:

1. The fraction of intrinsic $\mathrm{C}$ IV systems in our sample NALs is $11 \%-19 \%$. This value increases to $33 \%$ if only associated systems, within $5000 \mathrm{~km} \mathrm{~s}^{-1}$ of the quasar emission redshift, are considered. This is roughly consistent with previous studies of associated $\mathrm{C}$ iv NALs at different redshifts and employing different methods. It is important to note, however, that all of the above fractions are, in fact, lower limits to the true fractions. The fraction of intrinsic Si Iv systems is $14 \%-18 \%$, although all of these are found in the nonassociated regions of the spectra, at offset velocities $v_{\text {shift }}<-5000 \mathrm{~km} \mathrm{~s}^{-1}$ relative to the quasar redshifts. Severe contamination by the Ly $\alpha$ forest allowed us to search only for associated $\mathrm{N} v$ systems. We found $75 \%$ of such systems to be reliably intrinsic.

2. The minimum fraction of quasars that have one or more intrinsic NALs is $43 \%-54 \%$. While C Iv intrinsic NALs were detected in $24 \%-32 \%$ of the quasars with adequate velocity coverage, $\mathrm{N} v$ intrinsic NALs were detected in $19 \%$ of the quasars, and $\mathrm{Si}$ IV intrinsic NALs in $19 \%-24 \%$ of the quasars. These are lower limits because our spectra do not have full offset velocity coverage for these transitions and because some intrinsic absorbers may not exhibit the signature of partial coverage. This places a constraint on the solid angle subtended by the absorber to the background source(s).

3 . We find that $10 \%-17 \%$ of nonassociated C Iv NAL systems are intrinsic. In cosmological applications, nonassociated systems are typically taken to be intervening. Thus, our result is important because it shows that it is necessary to correct derived cosmological quantities for a contamination by intrinsic NALs. A similar conclusion was reached by Richards et al. (1999) and Richards (2001) based on a statistical study of NALs in different quasar samples, although they estimated a somewhat higher contamination of $\sim 36 \%$. Taking the two estimates at face value (i.e., neglecting the possibility that they differ because of systematic effects and demanding that they should be reconciled), we are led to the conclusion that only $30 \%-50 \%$ of intrinsic $\mathrm{C}$ IV NALs exhibit partial coverage.

4. The coverage fractions of intrinsic NALs in our sample span almost the entire range from 0 to 1 . Since there is a range of sizes for intrinsic gas parcels, this also implies that there are a significant number of intrinsic absorbers that have full coverage and thus were not detected in our survey. There is no apparent relationship between coverage fraction and velocity offset, nor is there any relation between the NAL properties or frequency of incidence and the properties of the host quasars (such as optical or radio luminosity).

5. We consider the ionization structure of the 39 class $A$ and B intrinsic NAL systems in our sample and find two major categories, which may represent absorbers of different densities and/or at different distances from the source of the ionizing continuum:

a) "Strong $\mathrm{C}$ IV" systems are characterized by strong, partially covered $\mathrm{C}$ Iv doublets, strong, usually "black," Ly $\alpha$ lines, and relatively weak or undetectable $\mathrm{N} v$ doublets. Of the 
28 systems in this category, 25 also have intermediate-ionization lines detected (such as Si III, C III, and/or Si Iv) and 15 systems also have low-ionization absorption detected (e.g., O I, Si II, and $\mathrm{Al} \mathrm{II}$ ). In cases where the Ly $\alpha$ profile is black, it is clear that it does not arise in the same gas parcel as the C IV absorption, since the coverage fraction for $\operatorname{Ly} \alpha$ is clearly 1 . These $\mathrm{C}$ IV systems cannot be distinguished from typical intervening systems except by partial coverage.

b) "Strong N v" systems are characterized by strong $\mathrm{N} \mathrm{v}$ lines and relatively weak, nonblack $\operatorname{Ly} \alpha$ lines (with less than twice the equivalent width of their $\mathrm{N} v$ lines). $\mathrm{C}$ IV and $\mathrm{O}$ VI lines may also be detected in these systems, and in some cases $\mathrm{O}$ vi may be stronger than $\mathrm{N} v$. We find 11 systems in this way, 5 of which have intermediate-ionization transitions detected, and only 1 of which has low-ionization transitions detected.

6. About $53 \%$ of class A or B NAL systems include lowionization lines. This fraction is much higher than that of LoBAL quasars among all BAL quasars $(13 \%-17 \%)$. In the $15 \mathrm{C}$ IV systems with detected low-ionization lines, the line profiles of the $\mathrm{C}$ IV and low-ionization lines are similar. In particular, lowionization lines are rarely detected at velocities other than those of the partially covered C IV components, implying that both families of lines arise in the same parcels of gas.

7. Our detection of a significant population of intrinsic, highvelocity NALs $\left(v_{\text {shift }} \sim 10^{4} \mathrm{~km} \mathrm{~s}^{-1}\right)$ disfavors scenarios in which the absorbing gas is associated with a pressure-driven wind that does not originate very deep in the potential well of the black hole. The low-velocity NALs that we have detected could still originate in pressure-driven winds. However, economy of means leads us to prefer accretion disk winds because these can explain both the high- and the low-velocity NALs.

Repeated observations of the NALs in this sample at the same spectral resolution and $\mathrm{S} / \mathrm{N}$ would be particularly valuable for the following reasons. First, they will allow us to search for NAL variability, which can be used to confirm intrinsic NALs and to probe the relation between variability and partial coverage of intrinsic NALs. Second, variability sampled via multiepoch observations carries additional information that may allow us to constrain properties of the absorber (e.g., density and distance from the continuum source; see applications by Hamann et al. 1997b; Narayanan et al. 2004; M05). Multiepoch observations of such a large sample of NALs have never been carried out, in spite of their utility in providing crucial constraints on quasar outflows. The sample of NALs presented here is an ideal starting point for such work.

This work was supported by NASA grant NAG5-10817. We would like to thank Christopher Churchill for providing us with the MINFIT software package. UCSD work was supported in part by NASA grant NAG5-13113 and NSF grant AST 0507717. We also thank the anonymous referee for very useful comments and suggestions.

\section{APPENDIX}

\section{DATABASE OF NAL SYSTEMS}

In this appendix we present the detailed information on the metal line systems detected in our spectra. The results of model fits to the profiles of NALs found in our spectra are listed in Table 5. In each block of this table, we list the properties of a different Poisson system derived from profile fits, as described in $\S 3.1$. The first line of each block gives the properties of the Poisson system as a whole, including the identification, mean observed wavelength, and total observed equivalent width. The following lines of the block give information on individual kinematic components that make up this Poisson system. Included are also NALs that do not meet the restframe equivalent width criterion for our complete sample (i.e., they have $W_{\text {rest }}<W_{\min }$ ).

Columns (1) and (2) of Table 5 give the quasar name ${ }^{12}$ and emission redshift. Since the true systemic redshift could be larger than this value (see discussion in $\S 2$ ), the velocity offsets relative to the systemic redshift could have large uncertainties. However, the uncertainties of the velocity offsets relative to the frame of the broad emission lines are rather small. If a line is detected within $5000 \mathrm{~km} \mathrm{~s}$ of the quasar, it is identified in column (3) as an AAL. The ion name and the observed wavelength are listed in columns (4) and (5). Columns (6) and (7) give the observed equivalent width and absorption redshift. The velocity offset of this component from the quasar is given in column (8). Columns (9) and (10) denote column density and Doppler parameter derived from the profile fit, along with $1 \sigma$ errors. The coverage fraction with its $1 \sigma$ error (which includes both Poisson noise errors and the error from placement of the continuum level) is listed in column (11). If an unphysical value of $C_{f}$ (i.e., $C_{f}<0$ or $C_{f}>1$ ) is given by MINFIT for some components, we refit the absorption profile again, assuming $C_{f}=1$ for these components as described in $\S 3.1$. Column (12) gives an assessment of the reliability of a physical association of the absorber with the quasar (discussed in $\S 3.1$ ). Column (13) gives the membership in specific subsamples, as follows: $\mathrm{L} / \mathrm{Q}=$ radio-loud/radio-quiet subsample, $\mathrm{A}=$ associated NAL (within $5000 \mathrm{~km} \mathrm{~s}^{-1}$ of $\left.z_{\mathrm{em}}\right)$, and $\mathrm{H}=$ homogeneous/complete sample $\left[W_{\text {rest }} / \sigma\left(W_{\text {rest }}\right) \geq 5\right]$. Single lines identified at the same redshift as the doublets are presented in column (14), even if they are detected in the Ly $\alpha$ forest. The detection criteria for single lines are subjective.

The results of the coverage fraction analysis applied to $206 \mathrm{C}$ IV , N v, and Si IV doublets are shown graphically in Figure Set 12 . In these plots, velocities are defined relative to the flux-weighted center of the system, as given in the first line of each block of Table 5. Included in these plots are the profiles of the two members of each doublet, as well as the coverage fraction as a function of velocity, as determined by the two methods described in $\S 3.1$. If we had to assume $C_{f}=1$ for some components (see below), we do not plot $C_{f}$ values for them in these system plots. We also do not plot the results from MINFIT and/or the pixel-by-pixel method, if the $1 \sigma$ error is larger than 1 .

In Figure Set 13 we present the line profiles of important transitions of individual class A and class B systems on a common velocity scale. We refer to these figures in our discussion of individual class A and class B systems, which follows below:

HE 0130-4021 $\left(z_{\mathrm{em}}=3.030\right)$. - This quasar, discovered by Osmer \& Smith (1976) on Tololo survey plates, is a very blue object (Adam 1985). A faint radio source was also detected with $f_{\nu}(5 \mathrm{GHz})=4 \pm 3 \mathrm{mJy}$ (Smith \& Wright 1980). The X-ray luminosity at $2-10 \mathrm{keV}$ is estimated to be $8.28 \times 10^{45} \mathrm{ergs} \mathrm{s}^{-1}$ (Page et al. 2003).

${ }^{12}$ Quasar names are based on B1950.0 coordinates. 
TABLE 5

Properties of Narrow Absorption Lines

\begin{tabular}{|c|c|c|c|c|c|c|c|c|c|c|c|c|c|}
\hline $\begin{array}{l}\text { QSO } \\
(1)\end{array}$ & $\begin{array}{l}z_{\mathrm{em}} \\
(2)\end{array}$ & $\begin{array}{l}\mathrm{AAL}^{\mathrm{a}} \\
(3)\end{array}$ & $\begin{array}{l}\text { Ion } \\
\text { (4) }\end{array}$ & $\begin{array}{c}\lambda_{\text {obs }}{ }^{b} \\
(\AA) \\
(5)\end{array}$ & $\begin{array}{c}W_{\mathrm{obs}}^{\mathrm{c}} \\
(\AA) \\
(6)\end{array}$ & $\begin{array}{l}z_{\mathrm{abs}}^{\mathrm{d}} \\
(7)\end{array}$ & $\begin{array}{c}v_{\text {shift }} \\
\left(\mathrm{km} \mathrm{s}^{-1}\right) \\
(8)\end{array}$ & $\begin{array}{c}\log N \\
\left(\mathrm{~cm}^{-2}\right) \\
(9)\end{array}$ & $\begin{array}{c}\sigma(v) \\
\left(\mathrm{km} \mathrm{s}^{-1}\right) \\
(10)\end{array}$ & $\begin{array}{l}C_{f}^{\mathrm{f}} \\
(11)\end{array}$ & $\begin{array}{c}\text { Reliability Class }{ }^{\mathrm{g}} \\
\text { (12) }\end{array}$ & $\begin{array}{l}\text { Sample }^{\mathrm{h}} \\
\text { (13) }\end{array}$ & $\begin{array}{l}\text { Other Ions }{ }^{\mathrm{i}} \\
\text { (14) }\end{array}$ \\
\hline \multirow[t]{12}{*}{ Q0004+1711......... } & 2.890 & & $\mathrm{C}$ IV & 4843.0 & 2.7768 & 2.1281 & -64375 & & 82.0 & & $\mathrm{C} 1$ & $\mathrm{~L}, \mathrm{H}$ & Ly $\alpha$ (C II $\lambda 1335$, Si III $\lambda 1207$, Si IV $)$ \\
\hline & 2.890 & $\ldots$ & $\mathrm{C}$ IV & 4843.0 & 2.7768 & 2.1271 & -64473 & $13.71 \pm 0.22$ & $19.3 \pm 2.9$ & $0.49_{-0.17}^{+0.34}$ & $\mathrm{C} 1$ & $\mathrm{~L}, \mathrm{H}$ & Ly $\alpha$ (C II $\lambda 1335$, Si III $\lambda 1207$, Si IV $)$ \\
\hline & 2.890 & $\ldots$ & $\mathrm{C}$ IV & 4843.0 & 2.7768 & 2.1275 & -64431 & $14.21 \pm 0.05$ & $11.9 \pm 0.7$ & $0.97_{-0.07}^{+0.07}$ & $\mathrm{C} 1$ & $\mathrm{~L}, \mathrm{H}$ & Ly $\alpha$ (C II $\lambda 1335$, Si III $\lambda 1207$, Si IV) \\
\hline & 2.890 & $\ldots$ & $\mathrm{C}$ iv & 4843.0 & 2.7768 & 2.1280 & -64385 & $14.48 \pm 0.04$ & $22.1 \pm 1.6$ & $0.98_{-0.07}^{+0.07}$ & $\mathrm{C} 1$ & $\mathrm{~L}, \mathrm{H}$ & Ly $\alpha$ (C II $\lambda 1335$, Si III $\lambda 1207$, Si Iv) \\
\hline & 2.890 & $\ldots$ & $\mathrm{C}$ iv & 4843.0 & 2.7768 & 2.1283 & -64357 & $12.44 \pm 1.80$ & $7.4 \pm 13.4$ & 1.00 & $\mathrm{C} 1$ & $\mathrm{~L}, \mathrm{H}$ & Ly $\alpha$ (C II $\lambda 1335$, Si III $\lambda 1207$, Si Iv) \\
\hline & 2.890 & $\ldots$ & $\mathrm{C}$ iv & 4843.0 & 2.7768 & 2.1286 & -64334 & $14.28 \pm 0.05$ & $15.3 \pm 3.3$ & 1.00 & $\mathrm{C} 1$ & $\mathrm{~L}, \mathrm{H}$ & Ly $\alpha$ (C II $\lambda 1335$, Si III $\lambda 1207$, Si IV $)$ \\
\hline & 2.890 & $\ldots$ & $\mathrm{C}$ IV & 4843.0 & 2.7768 & 2.1289 & -64309 & $12.74 \pm 1.32$ & $11.0 \pm 13.1$ & 1.00 & $\mathrm{C} 1$ & $\mathrm{~L}, \mathrm{H}$ & Ly $\alpha$ (C II $\lambda 1335$, Si III $\lambda 1207$, Si IV $)$ \\
\hline & 2.890 & $\ldots$ & $\mathrm{C}$ IV & 4843.0 & 2.7768 & 2.1290 & -64298 & $13.88 \pm 0.06$ & $10.8 \pm 0.7$ & $1.00_{-0.07}^{+0.08}$ & $\mathrm{C} 1$ & $\mathrm{~L}, \mathrm{H}$ & Ly $\alpha$ (C II $\lambda 1335, \mathrm{Si}_{\text {III }} \lambda 1207, \mathrm{Si}$ IV $)$ \\
\hline & 2.890 & $\ldots$ & $\mathrm{Si}$ IV & 4903.0 & 1.0584 & 2.5178 & -30068 & $\ldots$ & 42.6 & $\ldots$ & $\mathrm{C} 1$ & $\mathrm{~L}, \mathrm{H}$ & Ly $\alpha$ (Si пा $\lambda \lambda 1190,1193, \mathrm{Si}$ пा $\lambda 1260, \mathrm{Si}$ ІाI $\lambda 1207, \mathrm{Si}$ v) \\
\hline & 2.890 & $\ldots$ & $\mathrm{Si}$ IV & 4903.0 & 1.0584 & 2.5171 & -30132 & $12.30 \pm 0.12$ & $2.7 \pm 2.9$ & 1.00 & $\mathrm{C} 1$ & $\mathrm{~L}, \mathrm{H}$ & Ly $\alpha$ (Si пा $\lambda \lambda 1190,1193, \mathrm{Si}$ пा $\lambda 1260, \mathrm{Si}$ пा $\lambda 1207, \mathrm{Si}$ v $)$ \\
\hline & 2.890 & $\ldots$ & Si IV & 4903.0 & 1.0584 & 2.5177 & -30082 & $13.97 \pm 0.07$ & $11.5 \pm 0.5$ & $0.99_{-0.09}^{+0.09}$ & $\mathrm{C} 1$ & $\mathrm{~L}, \mathrm{H}$ & Ly $\alpha$ (Si II $\lambda \lambda 1190,1193$, Si II $\lambda 1260, \mathrm{Si}$ III $\lambda 1207, \mathrm{Si}$ Iv) \\
\hline & 2.890 & $\ldots$ & Si IV & 4903.0 & 1.0584 & 2.5182 & -30036 & $13.52 \pm 0.07$ & $11.4 \pm 0.6$ & $0.85_{-0.09}^{+0.09}$ & $\mathrm{C} 1$ & $\mathrm{~L}, \mathrm{H}$ & Ly $\alpha$ (Si II $\lambda \lambda 1190,1193, \mathrm{Si}$ Іा $\lambda 1260, \mathrm{Si}$ III $\lambda 1207, \mathrm{Si}$ Iv) \\
\hline
\end{tabular}

Notes.-Table 5 is published in its entirety in the electronic edition of the Astrophysical Journal Supplement. A portion is shown here for guidance regarding its form and content

${ }^{a}$ Associated absorption line, $\left|v_{\text {shiff }}\right| \leq 5000 \mathrm{~km} \mathrm{~s}^{-1}$

Wavelength of flux-weighted line center.

c Observed frame equivalent width of blue member.

${ }^{\mathrm{d}}$ Redshift of flux-weighted line center.

Velocity offset from quasar emission redshift.

Coverage fraction.

Partial coverage reliability classes, as defined in $\S 3.1$

${ }^{\text {h }}$ Subsamples: $\mathrm{L}=$ radio-loud, $\mathrm{Q}=$ radio-quiet, $\mathrm{A}=$ associated (within $5000 \mathrm{~km} \mathrm{~s}^{-1}$ of $z_{\text {em }}$ ), and $\mathrm{H}=$ homogeneous/complete sample with $W_{\text {rest }} / \sigma\left(W_{\text {rest }} \geq 5\right.$ ).

Other lines that are detected in the system. Lines in parentheses are in Ly $\alpha$ forest, i.e., less reliable. 


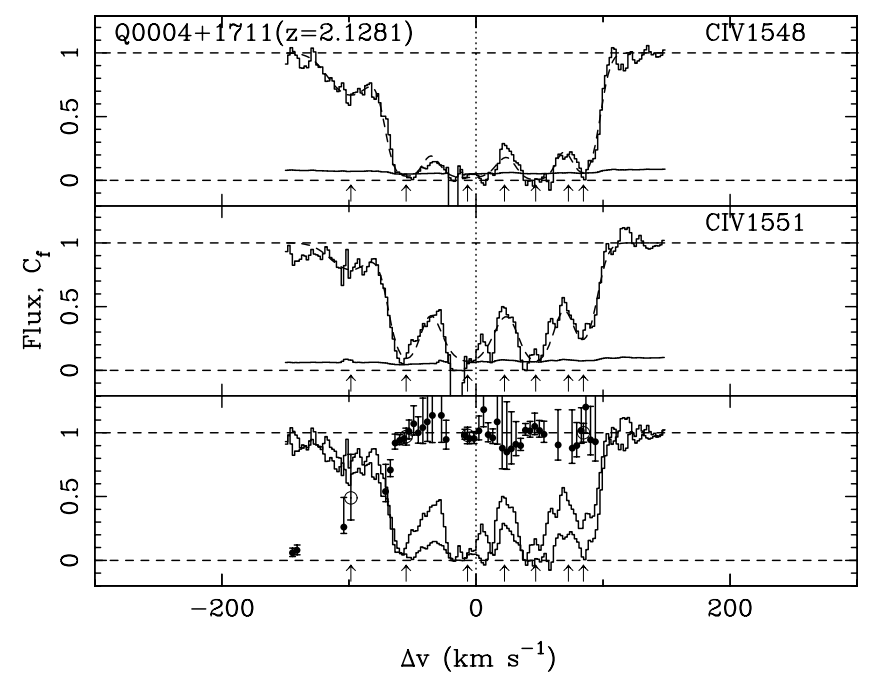

FIG. 12.1. Q0004+1711 $(z=2.1281, \mathrm{C}$ IV $)$

Fig. SEт 12.-Results of partial coverage analysis applied to $206 \mathrm{C} \mathrm{Iv}, \mathrm{N}$ v, and Si iv doublets. Velocities are defined relative to the flux-weighted center of the system, as given in the first line of each block of Table 5 . The top and middle panels show the profiles of the blue and red members of a doublet on a common velocity scale, with the model profile produced by MINFIT superposed as a dashed line. The positions of the kinematic components making up the model are marked with upward arrows in the bottom of each panel. The bottom panel shows the two profiles together, along with the resulting coverage fractions and their $1 \sigma$ error bars. The coverage fraction determined by the pixel-by-pixel method is plotted in the form of filled circles, while the coverage fraction determined by the fitting method is plotted in the form of open circles. [See the electronic edition of the Supplement for color Figs. 12.2-12.206.]

$z_{\mathrm{abs}}=2.2316$ (class $B$ ). - This system contains four C IV components, of which two at $\Delta v=-33$ and $6 \mathrm{~km} \mathrm{~s}^{-1}$ show possible partial coverage, $C_{f}=0.83+0.10-0.09$ and $0.80+0.16-0.15$. The $C_{f}$ value of the component at $\Delta v=-69 \mathrm{~km} \mathrm{~s}^{-1}$ is not reliable because it is located in the wing of the adjacent component. The velocity plots for various transitions are presented in Figure 13.1. We found an Si Iv doublet in this system, although it is not listed in our table because it blends with the Ly $\alpha$ forest. The lines in the $\mathrm{Al}$ II $\lambda 1671$ window cannot be $\mathrm{Al}$ II because there are no absorption lines at the same velocity for other low-ionization transitions. The Ly $\alpha$ profile is saturated at the velocity of the $\mathrm{C}$ IV components, which is similar to typical intervening absorption systems. We classify the system into class B because two components have $C_{f}$ values smaller than 1 by more than $1 \sigma$. We would classify this system as a $1-\mathrm{HI}$ system.

$z_{\mathrm{abs}}=2.5597$ (class $A$ ). - This $\mathrm{C}_{\text {IV }}$ system consists of seven separate components. We found that the three components at $\Delta v=$ 5,27 , and $51 \mathrm{~km} \mathrm{~s}^{-1}$ have partial coverage in both the MINFIT and pixel-by-pixel analysis. For three components at $\Delta v<0 \mathrm{~km} \mathrm{~s}^{-1}$, reliable fits could not be obtained since the $\mathrm{C}$ IV doublet ratio is greater than 2 . Blue members of these components could be blended with other lines. The velocity plots for various transitions are presented in Figure 13.2. The Ly $\alpha$ profile is unsaturated at negative velocities, which is unusually weak for a $\mathrm{C}$ IV system, and it follows the kinematics of $\mathrm{C}$ iv quite closely. The low-ionization transitions Si II $\lambda 1527$ and $\mathrm{Al}$ II $\lambda 1671$ are not detected in clean areas of the spectrum, but all other low-ionization transitions suffered from possible blends with the Ly $\alpha$ forest and could not be interpreted. Si IV is clearly detected for the component at $75 \mathrm{~km} \mathrm{~s}^{-1}$ but cannot be analyzed for partial coverage due to a data defect in the $\lambda 1394$ profile. $\mathrm{N} v$ is covered and is not detected, although the region around the $75 \mathrm{~km} \mathrm{~s}^{-1}$ component is affected by blending. The possibilities of uncertainty in the continuum fit at the position of $\mathrm{C}$ IV and of blends in the $\mathrm{C}$ IV profile suggest that this system may not be intrinsic. On the other hand, the weakness of Ly $\alpha$ relative to $\mathrm{C}$ IV at negative velocities implies unusual ionization conditions and/or metallicity for an intervening absorber. The similarity between the Ly $\alpha$ and $\mathrm{C}$ IV $\lambda 1551$ profiles as compared to $\mathrm{C}$ iv $\lambda 1548$ suggests that a blend may be affecting the latter. This could explain the discrepancy in the doublet ratio in the negative velocity region so that that region would also be consistent with our intrinsic classification derived for positive velocities. In this case it would be a 1-HI system.

$z_{\mathrm{abs}}=2.9749$ (class A).-This is an associated system (i.e., the velocity offset relative to the quasar emission redshift is smaller than $5000 \mathrm{~km} \mathrm{~s}^{-1}$ ). The smooth line profile of $\mathrm{N} \mathrm{v}$ suggests that it may be an intrinsic system. We detected at least nine narrow components, whose $C_{f}$ values by MINFIT analysis are all significantly less than 1, except for one component, and almost perfectly agree with the results of pixel-by-pixel analysis. The system is plotted in velocity space in Figure 13.3. The two "black" lines at the center of the Ly $\alpha$ window are not Ly $\alpha$ but $\mathrm{C}$ IV $\lambda 1548$ at $z_{\mathrm{abs}} \sim 2.121$. Over much of the velocity window, the Ly $\alpha$ absorption is similar in strength to the $\mathrm{N}$ v. The $\mathrm{O}$ I and $\mathrm{C}$ II lines are not detected at a $5 \sigma$ level. The absorption profile seen in the Si II $\lambda 1260$ window cannot be $\mathrm{Si}$ II because other low-ionization transitions are not detected. The $\mathrm{C}$ IV doublet is not covered in our spectrum. Complex and strong $\mathrm{O}$ VI is detected, but it is in the Ly $\alpha$ forest so that blends render $C_{f}$ analysis impossible. This system is similar to an intrinsic NAL system at $z_{\text {abs }} \sim 0.11$ toward the quasar RX J1230.8+0115, in which very strong $\mathrm{N} v$ and $\mathrm{O}$ vI lines and relatively weak Ly $\alpha$ are detected (Ganguly et al. 2003). We consider this system as a typical example of a 2-H system.

Q0241-0146 ( $\left.z_{\mathrm{em}}=4.040\right)$. - The emission lines of this quasar, such as Ly $\alpha, \mathrm{O}$ I, C II, Si IV, and C IV, are known to be very broad and rounded (Storrie-Lombardi et al. 1996). The rest-frame equivalent width, calculated by direct integration of the $\mathrm{C}$ IV emission line, is $27 \AA$ (Constantin et al. 2002). A radio source is not detected to a limit of $2.5 \mathrm{mJy}$ at $1.4 \mathrm{GHz}$ in the course of the NRAO VLA Sky Survey (NVSS; Condon et al. 1998).

$z_{\mathrm{abs}}=3.0451$ (class $\left.B\right)$. - The single component of this $\mathrm{C}_{\mathrm{Iv}} \mathrm{NAL}$ system has a $C_{f}$ value smaller than 1 by both methods. Although the MINFIT value differs from 1 by just $3 \sigma$, the fitting model is discrepant in the wings. Perhaps another component is needed. Si IV 


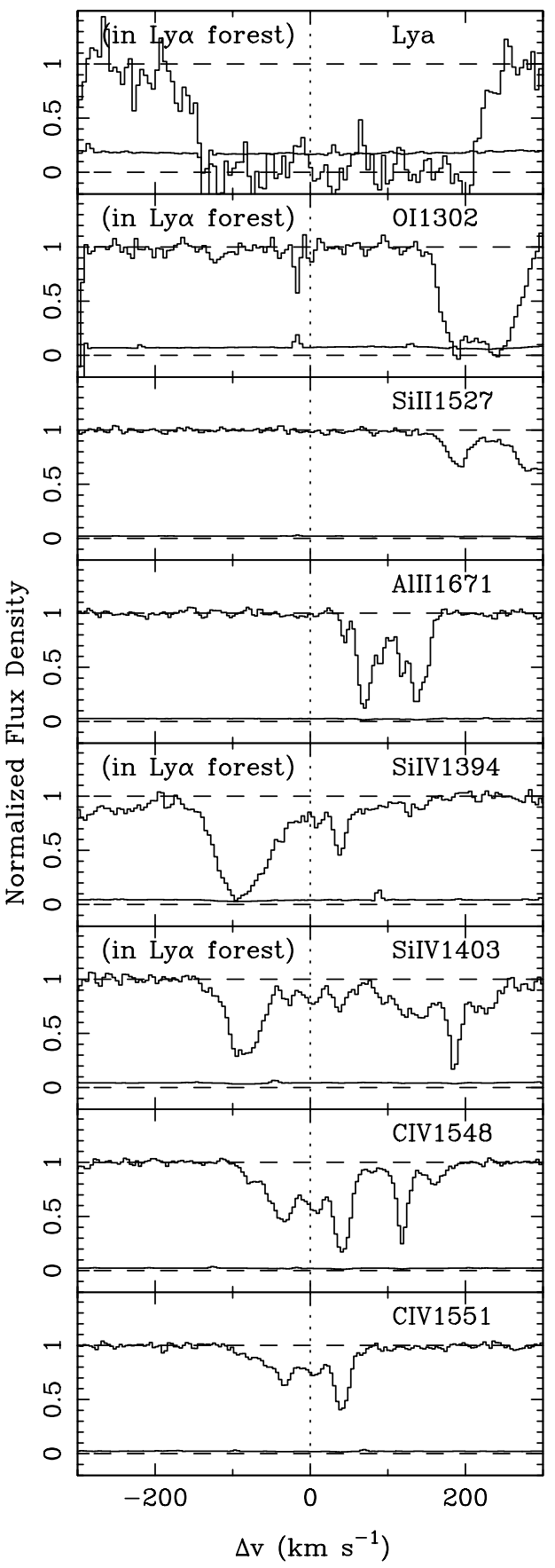

FIG. SET 13.- Velocity plots of various lines detected in the class B NAL system at $z_{\mathrm{abs}}=2.2316$ in HE 0130-4021. [See the electronic edition of the Supplement for Figs. 13.2-13.39.]

may also be detected in the Ly $\alpha$ forest. The velocity plots for various transitions are presented in Figure 13.4. At the velocity of the $\mathrm{C}$ IV component, the Ly $\alpha$ profile is saturated, similar to typical intervening absorbers. Although many transitions are covered, most are affected by blending with Ly $\alpha$ forest lines, and we have only weak constraints. In particular, there is no constraint on $\mathrm{N} v$ absorption due to blending with strong Ly $\alpha$ forest lines. Classification of this absorber as intrinsic or intervening is highly uncertain. We would classify this system as a 1-HI system.

HE 0322-3213 $\left(z_{\mathrm{em}}=3.302\right)$. - Coordinate of this quasar is given in Kirkman et al. (2005). We classify this quasar as a radio-quiet quasar because no radio source was detected within $10^{\prime \prime}$ of the optical source above the detection limit of $2.5 \mathrm{mJy}$ at $1.4 \mathrm{GHz}$ in the course of the NVSS.

$z_{\text {abs }}=3.2781$ (class $\left.A\right)$. - An associated N v NAL in this system has an asymmetric line profile, with a strong wing at the blue side. The line profile is also very smooth, which is a common feature of intrinsic N v NALs. $C_{f}$ is significantly less than 1 over most of the profile, and it changes substantially with velocity. This system has a high probability of being an intrinsic NAL. The system is plotted in velocity space in Figure 13.5. Although it falls partially on an order gap, the Ly $\alpha$ profile is clearly not "black" in the region of the $\mathrm{N} v$ absorption. Low-ionization absorption is poorly constrained, but the intermediate-ionization transition, Si III $\lambda 1207$, is not detected. C Iv is not covered. We classified this as a 2-H system. 
$z_{\mathrm{abs}}=3.2818(\operatorname{class} A)$. - The line profile of the $\mathrm{N} v \mathrm{NAL}$ in this associated system is very similar to the $\mathrm{N} v \mathrm{NAL}$ in the nearby system at $z_{\mathrm{abs}}=3.2781$ (a separation of $\sim 250 \mathrm{~km} \mathrm{~s}^{-1}$ ), suggesting that these two systems are closely related. This system also has an asymmetric profile, with an extended red wing. The $\mathrm{N} v$ doublet was adequately fitted with four components. For all four components (see Table 5), $C_{f}$ is substantially less than 1 as determined by both methods; thus, we are confident that this system is intrinsic. The velocity plots for various transitions related to this system are presented in Figure 13.6. Although stronger than the $\mathrm{N} v$ (and possibly affected by blending), it is clear that the Ly $\alpha$ absorption at the velocity corresponding to this system is not saturated. Although Si II, C II, and Si III are not detected for this system, there appears to be related C III absorption. The likelihood that it is real is increased by the great similarity between the kinematics of the $\mathrm{C}$ III and $\mathrm{N} v$ profiles. Related $\mathrm{O}$ VI appears to be detected as well, since the $\lambda 1032$ profile is similar in shape to $\mathrm{N} v$ but is badly blended in the Ly $\alpha$ forest. This system is classified as a $2-\mathrm{HI}$ system, with probable absorption in the intermediate-ionization transition, $\mathrm{C}$ III, as well as in O vI.

Q0450-1310 $\left(z_{\mathrm{em}}=2.300\right)$. - This quasar was discovered by Hazard (Sargent et al. 1988). We classify it as a radio-quiet quasar because no radio source was detected within $10^{\prime \prime}$ of the optical coordinates (NVSS).

$z_{\mathrm{abs}}=2.2307$ (class $A$ ). - This system has already been studied as an intrinsic NAL system (G99; Petitjean et al. 1994). By using the pixel-by-pixel method, G99 found that the coverage fractions of the C IV, N v, and Si IV lines are all similar to each other, but that they change with velocity across the absorption profiles. Here, in our partial coverage analysis, we investigated only Si IV lines because $\mathrm{C}$ IV is outside the observed wavelength region and $\mathrm{N} v$ is blended with the Ly $\alpha$ forest and so does not meet our sample criteria. Nonetheless, if we evaluated $C_{f}$ values for the $\mathrm{N} v$ doublet, we would find partial coverage. Detailed analysis of the $\mathrm{N}$ v doublet is presented in $\mathrm{J}$. Wu et al. (2007, in preparation). MINFIT gives $C_{f} \sim 0.69 \pm 0.06$ for the strongest Si IV component, which is similar to the result from pixel-by-pixel analysis in G99, $C_{f}=0.59-0.88$. The velocity plot of this system is presented in Figure 13.7. The fraction of the flux absorbed at negative velocities in the Ly $\alpha$ profile is $\sim 0.8-0.9$, which is a bit larger than $C_{f}$ of $\mathrm{Si}$ IV in the same region. The Ly $\alpha$ line is likely to be saturated there. On the other hand, the profile is black at positive velocities. Low-ionization transitions are not detected; however, Si III $\lambda 1207$ is detected with a profile very similar in shape to the Si IV. The $\mathrm{C}$ Iv profiles in G99 are strong, very similar to $\mathrm{N}$ v. The $C_{f}$ values of C IV and $\mathrm{N}$ v evaluated in G99 are almost unity in the positive velocity region. This $\mathrm{N} v$ intrinsic system appears to have two sets of components: lower ionization partial coverage components at $\Delta v<0 \mathrm{~km} \mathrm{~s}^{-1}$ and higher ionization full coverage components at $\Delta v>0 \mathrm{~km} \mathrm{~s}^{-1}$. We classify this system as a $2-\mathrm{HI}$ system.

$Q 0636+6801\left(z_{\mathrm{em}}=3.178\right)$. - This quasar is one of the most luminous quasars known, and it is listed as a radio-loud quasar in Hewitt \& Burbidge (1987).

$z_{\mathrm{abs}}=3.0134$ (class $\left.A\right)$. - The C IV NAL in this system, with $C_{f}$ value smaller than 1 for each of its two components, was also identified in Sargent et al. (1989). The line profile is relatively smooth. Although the C IV components are weak, leading to nonnegligible continuum fitting errors, the results of MINFIT and the pixel-by-pixel method agree, and the high S/N of the spectrum allows us to classify this system as class A. The velocity plot of this system is presented in Figure 13.8. The Ly $\alpha$ profile at this velocity is saturated and "black." A weak O vi doublet may be present, but the line profiles of the blue and red members do not match, indicating a blend, and it is hard to assess similarity with the $\mathrm{C}$ IV profiles. The $\mathrm{N} v$ doublet cannot be investigated because of blending with strong Ly $\alpha$ lines. This system is classified as a 1-HI intrinsic system.

Q0642+4454 $\left(z_{\mathrm{em}}=3.408\right)$. - This radio-loud quasar has been studied because it has a LLS (Carswell et al. 1975) with various metal absorption lines (Sargent et al. 1989). The core and extended flux densities at $5 \mathrm{GHz}$ in the rest frame are evaluated to be 104.4 and $1.8 \mathrm{mJy}$, respectively (Neff \& Hutchings 1990).

$z_{\text {abs }}=2.9721$ (class $A$ ). - Sargent et al. (1989) detected a C IV NAL in this system, which has at least four components that we find to have partial coverage. We measure a larger $C_{f}$ for the two blueward components; however, our results for the redward components are affected by a large continuum fitting error. We note that the evidence for partial coverage in the strongest component, $C_{f}=0.51 \pm 0.09$, is definitive. A velocity plot is presented in Figure 13.9. The Ly $\alpha$ profile is saturated in the region of interest. Low-ionization transitions are not detected, evidenced mainly by a limit on Si II $\lambda 1527$. We have a likely detection of Si IV but cannot fit it with MINFIT because of a data defect in the Si IV $\lambda 1403$ profile. $\mathrm{N} v$ is affected by blends but is apparently weaker than $\mathrm{C}$ IV if it is even detected. The $\mathrm{O}$ vi profile is too heavily affected by the forest to assess whether absorption is present. We classify this as a $1-\mathrm{HI}$ system.

Q0805+0441 $\left(z_{\mathrm{em}}=2.880\right)$. - This quasar is well known as the radio source 4C 05.34. Chen et al. (1981) performed the first detailed study of absorption lines in this quasar. The 6 and $20 \mathrm{~cm}$ Green Bank flux densities at low resolution are 350.0 and $505.0 \mathrm{mJy}$, respectively (Richards et al. 2001). The core-to-lobe ratio at $20 \mathrm{~cm}$ was also evaluated to be about 0.6.

$z_{\mathrm{abs}}=2.4544(\operatorname{class} A)$. - The C IV NAL in this system is fitted with two components. The $C_{f}$ value of the component at $\Delta v=$ $-2 \mathrm{~km} \mathrm{~s}^{-1}$ is evaluated to be $\sim 0.60+0.08-0.07$ by MINFIT, while $C_{f} \sim 1$ for the weaker component at $\Delta v=-30 \mathrm{~km} \mathrm{~s}{ }^{-1}$. The velocity plot of this system is presented in Figure 13.10. Ly $\alpha$ is saturated and black. Low-ionization (e.g., O I, Si II, Al II) lines are not detected in this system. Si IV may be detected, but the $\lambda 1403$ transition is too weak compared to $\lambda 1394$, so perhaps both are blends. $\mathrm{N} v$ is not detected and $\mathrm{O}$ VI is not covered by our spectrum. This system classifies as a 1-HI system.

$z_{\mathrm{abs}}=2.6517$ (class $\left.A\right)$. - The C IV NAL in this system can be fitted with a single component. C IV shows evidence for partial coverage, $C_{f}=0.43+0.15-0.12$. Although $\mathrm{Si}$ IV NAL is also detected, the $C_{f}$ value for it is consistent with 1 . The system is plotted in velocity space in Figure 13.11. Ly $\alpha$ is black. The low-ionization transition C II $\lambda 1335$ may be detected, but we cannot confirm this with any other low-ionization transitions. Si IV and N v are detected and have similar kinematics to C IV. Si III $\lambda 1207$ is so strong that it seems it may be blended with some stronger line; however, its kinematics closely matches the Si IV. This is a 1-HIL system, assuming that the $\mathrm{C}$ II detection is valid.

$z_{\text {abs }}=2.8589$ (class $\left.A\right)$. - This is an associated system containing $\mathrm{C}$ IV and N v NALs. Although MINFIT and the pixel-by-pixel method both find single components with partial coverage, the $\mathrm{C}$ IV $\lambda 1551$ profile is severely affected by a data defect, so the $\mathrm{C}$ IV result is uncertain. On the other hand, the fitting result for the $\mathrm{N} v$ doublet is reliable and gives partial coverage, significant at the $3 \sigma$ level. The velocity plot is presented in Figure 13.12 for various transitions. The Ly $\alpha$ profile is not black at the velocity of the strongest $\mathrm{C}$ IV and $\mathrm{N} v$ absorption. It is, however, black $\sim 50 \mathrm{~km} \mathrm{~s}^{-1}$ blueward of that position. No low- or intermediate-ionization 
transitions (particularly $\mathrm{C}$ II and $\mathrm{Si}$ IV) were detected; however, $\mathrm{O}$ VI is detected. Unfortunately, contamination in the Ly $\alpha$ forest prevents us from evaluating $C_{f}$ FOR $\mathrm{O}$ vI. The $\mathrm{Ly} \alpha$ and high-ionization transitions of this absorber suggest that it is intrinsic. We would classify this system as a $2-\mathrm{H}$ system.

HE 0940-1050 $\left(z_{\mathrm{em}}=3.080\right)$. - This quasar was discovered during the course of the Hamburg/CfA Bright Quasar Survey. This is a radio-quiet quasar because the radio source corresponding to the optical image was not detected at a $2.5 \mathrm{mJy}$ detection limit at $1.4 \mathrm{GHz}$ (NVSS).

$z_{\text {abs }}=2.8347$ (class B). - This system, which has been studied by Reimers et al. (1995), contains C Iv and Si Iv NALs. The Si IV NAL is classified as class B due to the small $C_{f}$ values of its two redward components. However, these components are badly blended with one another, and despite agreement between MINFIT and the pixel-by-pixel results, there is significant ambiguity in our assessment. This is amplified by the fact that the C Iv NAL shows full coverage over the same region. As shown in Figure 13.13, Ly $\alpha$ is saturated and black in this region and remains so redward of the C IV and Si IV NALs. Low-ionization absorption (particularly $\mathrm{C}_{\text {II) }}$ is not detected; however, $\mathrm{Si}$ III is stronger than Si IV and matches the Si IV kinematics. N v and O vi are too badly affected by blending for us to assess whether they are detected. Overall, we assess that this is a class B NAL and, if intrinsic, is likely to be a 1-HI system.

$Q 1009+2956\left(z_{\mathrm{em}}=2.644\right)$. - This quasar is famous as a target for determination of the $\mathrm{D} / \mathrm{H}$ ratio. Burles \& Tytler (1998) investigated the metal-poor absorption system at $z_{\mathrm{abs}}=2.504$ to obtain a $\mathrm{D} / \mathrm{H}$ ratio measurement. No radio source has been discovered at $1.4 \mathrm{mJy}$.

$z_{\text {abs }}=2.2533($ class $A)$. - $\mathrm{A} \mathrm{Si} \mathrm{Iv} \mathrm{component} \mathrm{at} \Delta v=15 \mathrm{~km} \mathrm{~s}^{-1}$ shows partial coverage, $C_{f}=0.86 \pm 0.03$, with more than $3 \sigma$ reliability. Although this component blends with the adjacent component, the fitting result is reliable because of the high quality of this spectrum. Also, the results from the fitting method are consistent with results from pixel-by-pixel analysis at the center of the component. Figure 13.14 presents the velocity plots for several transitions. The strong line at $\Delta v \sim 90 \mathrm{~km} \mathrm{~s}^{-1}$ in the Si IV $\lambda 1394$ window is $\mathrm{N} \vee \lambda 1403$ at $z_{\mathrm{abs}}=2.6495$. However, this line does not greatly affect the Si Iv doublet because these lines are blends only in their weak wings. Our fitting analysis is still reliable. Unfortunately, the C IV doublet is not covered by our spectrum. Apparently, the $\mathrm{N} v$ doublet is not detected, and we cannot determine whether $\mathrm{O}$ vI is detected. Low- and intermediate-ionization transitions such as C II, Si II, and Si III are also identified. Ly $\alpha$ is saturated in this system. If this is an intrinsic system, it would be classified as a 1-HIL system, or possibly an $\mathrm{O}$ VI system if $\mathrm{O}$ vi absorption is strong.

$z_{\mathrm{abs}}=2.6495$ (class $A$ ). - The N v NAL in this associated system has a non-Gaussian "triangular" line profile, indicating that more than a one-component fit is needed. We fit the profile with three components at almost the same redshift. The $C_{f}$ values at $\Delta v=-4$ and $3 \mathrm{~km} \mathrm{~s}^{-1}$ are $C_{f} \sim 0.6-0.7$, similar to the results from the pixel-by-pixel method. The velocity plot of this system is presented in Figure 13.15. It is noteworthy that the $\operatorname{Ly} \alpha$ line is considerably weaker than the $\mathrm{N} v$, which is an indication of partial coverage. The $C_{f}$ of the $\operatorname{Ly} \alpha$ and $\operatorname{Ly} \beta$ lines is evaluated to be $0.32 \pm 0.02$ for the strongest component, which is smaller than the $C_{f}$ for $\mathrm{N}$ v. The $C_{f}$ is ion dependent in this system. However, as described in the previous section, the red member of this $\mathrm{N} \mathrm{v}$ doublet is blended with the blue member of the $\mathrm{Si}$ iv doublet at $z_{\mathrm{abs}}=2.2533$. The blend with the $\mathrm{N} \mathrm{v} \lambda 1243$ component causes our estimate of $C_{f}$ to be small compared to the actual value. However, based on the strength of the Si IV $\lambda 1403$ component, the blend should not be making a very large contribution, and the true $C_{f}$ value is very likely to be less than 1 . We classify this system as an intrinsic NAL candidate because of the $C_{f}$ values from $\mathrm{N} v$ and the line series and because it shows line locking. There are possible detections of C III $\lambda 977$ and Si III $\lambda 1207$, although Si III may not be real and at least has a blend to the blue. Unfortunately, C IV is not covered in our spectrum, and $\mathrm{O}$ vi may be detected but is too contaminated by the Ly $\alpha$ forest to provide any meaningful constraint. This is a good example of a 2-HI system, with weak Ly $\alpha$. It is also important to note that this system is redward of the quasar emission redshift.

Q1017+ $1055\left(z_{\mathrm{em}}=3.156\right)$. - Sargent et al. (1989) found BAL-type features in C IV and Si IV at $z_{\mathrm{abs}}=2.9720$, although there was no BAL-type profile in the Ly $\alpha$ line.

$z_{\mathrm{abs}}=2.5408$ (class B).- This system, originally identified in Sargent et al. (1989), contains three C iv NALs at $z_{\mathrm{abs}}=2.5392$, 2.5408, and 2.5425. The NAL at $z_{\mathrm{abs}}=2.5408$ is fitted with five components, the strongest of which shows apparent partial coverage with $C_{f}=0.72 \pm 0.10$. The two redward components may show partial coverage, but their $C_{f}$ values are significantly affected by possible errors in continuum fitting. The velocity plots for various transitions are presented in Figure 13.16. Lowionization lines, such as $\mathrm{Si}$ II and $\mathrm{Al}$ II, are not detected, despite the strong Ly $\alpha$ line. Weak Si III and Si IV lines may be present but are hard to confirm and separate from the Ly $\alpha$ forest. Although both $\mathrm{N} v$ transitions are badly affected by blends, using both we find that $\mathrm{N} v$ is not detected for this system. If intrinsic, this would be a 1-H system. However, the unphysical value for one of the components in the $z_{\mathrm{abs}}=2.5408 \mathrm{NAL}$, combined with the gradient in $C_{f}$ across this NAL, suggests a possible error in the slope of the continuum in this region.

$z_{\text {abs }} \sim 2.97$ (class $A$ ). - Sargent et al. (1989) detected this (mini-)BAL system. Both the $\mathrm{C}$ IV and Si Iv NALs have such broad line profiles (FWHM $>1000 \mathrm{~km} \mathrm{~s}^{-1}$ ) that their blue and red components are self-blended. Because of this, we cannot determine $C_{f}$ by either method. Nonetheless, because of the broad profiles, we classify the system as class A intrinsic. The velocity plot for this system is presented in Figure 13.17. We do not have any information on Ly $\alpha$ because the broad components have been "normalized out" in the Ly $\alpha$ forest. Similar problems arise in the regions of $\mathrm{N} v$ and O vi. Therefore, we plot the spectrum before normalization for these transitions. Along with the Si IV and C IV, C II $\lambda 1335$ with a broad line profile is also found. This system is noteworthy because it is so broad and because of the detection of the low-ionization $\mathrm{C}_{\text {II }}$ transition. We would classify this system as a 2-HIL miniBAL system.

$z_{\text {abs }} \sim 3.03$ (class A).-This is also a broad and smooth absorption line system, detected only in C Iv and not in Si iv. Again, $C_{f}$ cannot be measured due to self-blending, but the broad profile is used to classify the system as class A. The velocity plot of this system is presented in Figure 13.18. We do not have any information on $\mathrm{Ly} \alpha, \mathrm{N}$ v, and $\mathrm{O}$ vi because they have been normalized out. Again, for these transitions we plot the spectrum before normalization. No low- or intermediate-ionization transitions are detected; however, many of them have noisy coverage or blends so it is difficult to tell. This system could be a 2-H mini-BAL system. 
$z_{\text {abs }} \sim 3.11(\operatorname{class} A)$. - A broad self-blended N v profile, with a flux-weighted Doppler parameter of $\sim 1000 \mathrm{~km} \mathrm{~s}{ }^{-1}$, is seen in this associated system. Again, $C_{f}$ cannot be evaluated, but we consider this system to be class A intrinsic because of the broad profile. The velocity plot of this system is presented in Figure 13.19. Again, we can gain no information about Ly $\alpha$ or $\mathrm{O}$ vi in the Ly $\alpha$ forest region, and C IV was not covered in our spectrum. However, very narrow Si III and Si IV lines are detected at $z_{\mathrm{abs}}=3.1117$ and 3.1125 , and $\mathrm{C}_{\text {II }} \lambda 1335$ may be detected from the $z_{\mathrm{abs}}=3.1125$ system as well. The Ly $\alpha$ and Ly $\beta$ profiles are saturated in the region centered around these narrow components. Si II $\lambda 1260, \mathrm{C}_{\text {II }} \lambda 1335$, and O I $\lambda 1302$ may also be detected $150 \mathrm{~km} \mathrm{~s}{ }^{-1}$ redward of the narrow Si III and Si IV lines, but these identifications cannot be confirmed. The low- and intermediate-ionization transitions could arise in a system independent of the broad intrinsic $\mathrm{N} v$ system. We would classify the system as a 2-HI mini-BAL system with intermediate-ionization narrow lines.

Q1055+4611 $\left(z_{\mathrm{em}}=4.118\right)$. - Based on the optical and submillimeter data for this quasar, Isaak et al. (2002) inferred that the black hole mass, its accretion rate, and the dust mass around the quasar are $19.0 \times 10^{9} M_{\odot}, 420 M_{\odot} \mathrm{yr}^{-1}$, and $1.8 \times 10^{9} M_{\odot}$, respectively. The $\mathrm{X}$-ray flux at $2 \mathrm{keV}$ is $f_{\nu}(2 \mathrm{keV})=2.34 \times 10^{-31} \mathrm{ergs} \mathrm{cm}^{-2} \mathrm{~s}^{-1} \mathrm{~Hz}^{-1}$, and the X-ray (2 keV) to optical (2500 $\AA$ ) flux ratio was estimated to be $\alpha_{\text {ox }}=1.638_{-0.207}^{+0.228}$ (Bechtold et al. 2003).

$z_{\mathrm{abs}}=3.3658$ (class $B$ ). - If this is a real C IV doublet, the blueward member would coincide with the C IV $\lambda 1551$ line of a possible $\mathrm{C}$ IV doublet at $z_{\mathrm{abs}}=3.3591$. That possible doublet has an unphysical doublet ratio over much of its profile, indicating an unknown blend with the $\lambda 1548$ line. The redward member of this system would be blended with Si IV $\lambda 1403$ at $z_{\text {abs }}=3.8259$. However, that Si IV $\lambda 1403$ line already appears to be somewhat weak in comparison with its $\lambda 1394$ counterpart. These factors call into question the reality of this $z_{\mathrm{abs}}=3.3658$ system. Due to the several blends, it is impossible to evaluate $C_{f}$ reliably for any of the components. It is worth noting, however, that if this system exists, it exhibits line locking with the $z_{\mathrm{abs}}=3.3591$ system (a weak system, but confirmed with $\operatorname{Ly} \alpha$ line), which might suggest an intrinsic nature. It is because of the possible line locking that this system is classified as class B. The velocity plots for various transitions are presented in Figure 13.20. The Ly $\alpha$ in the region of interest is quite weak, similar in equivalent width to the $\mathrm{C}$ IV. This may be hard to explain, which also calls into question the reality of the doublet itself. On the other hand, we have not been able to determine other feasible identifications for these lines, which are not in the Ly $\alpha$ forest. We would classify this system as a 1-HI system. This is one of the least certain class B systems in our sample. $z_{\mathrm{abs}}=3.5314($ class $A)$. - The Si IV NAL in this system has a complex line profile. The strong components at $\Delta v<0 \mathrm{~km} \mathrm{~s}$ show full coverage. Two of the weaker components at $\Delta v>0 \mathrm{~km} \mathrm{~s}^{-1}$ show partial coverage. Although they are weak components, uncertainties of $C_{f}$ values from the continuum fitting errors are very small because their $C_{f}$ values are much smaller than 1.0 (see $\S 3.1$ ). The system is plotted in velocity space in Figure 13.21. The Ly $\alpha$ absorption is very strong for this system. Because of confusion in the Ly $\alpha$ forest, no other transitions but C II $\lambda 1335$, Si II $\lambda 1193$, and Si III $\lambda 1207$ can be claimed as detections. C IV is not covered. The $\mathrm{N} v$ doublet might be detected at certain velocities but is too badly contaminated in the Ly $\alpha$ forest to tell. Therefore, we tentatively classify this as a 1-HIL system.

HS 1103+6416 $\left(z_{\mathrm{em}}=2.191\right)$. - This quasar was discovered during the Hamburg Quasar Survey (Hagen et al. 1995). A LLS at $z_{\text {abs }} \sim 1.89$, with an estimated optical depth $\tau \sim 2$ at the limit, was identified (Reimers et al. 1995).

$z_{\text {abs }}=1.8874$ (class $\left.B\right)$. - This C IV NAL, containing three narrow components, could be line locked with a C IV NAL at $z_{\text {abs }}=$ 1.8919. However, the overlap could also be attributed to clustering of $\mathrm{C}$ IV absorbers on a scale of $\sim 500 \mathrm{~km} \mathrm{~s}{ }^{-1}$. Because of the blending of the $\mathrm{C}$ IV $\lambda 1551$ profile with the much stronger $\lambda 1548$ profile from the $z_{\mathrm{abs}}=1.8919$ system, a $C_{f}$ analysis cannot be performed. The velocity plots for various transitions are presented in Figure 13.22. The Ly $\alpha$ profile is strong and saturated. Weak Si III $\lambda 1207$ and Si IV are detected, but low-ionization transitions, most notably C II, are not. N v is also not detected. Other than the possible line locking, this would appear to be a normal intervening absorber. We would classify this system as a 1-HI system.

$z_{\mathrm{abs}}=1.8919$ (class $\left.B\right)$. - This strong C IV NAL can be fitted with seven components based on just C IV $\lambda 1551$. The $\mathrm{C}$ IV $\lambda 1548$ of two components at $\Delta v=45$ and $66 \mathrm{~km} \mathrm{~s}^{-1}$ is strongly blended with the $\mathrm{C}$ IV $\lambda 1551$ from the $z_{\text {abs }}=1.8874$ system (possible line locking). A strong $\mathrm{Si}$ IV doublet is also detected for this system. $C_{f}$ values could be derived for four components of the Si IV doublet, but three of them are consistent with full coverage within 2-3 $\sigma$ errors. The velocity plots for various transitions are presented in Figure 13.23. The Ly $\alpha$ related to this system is strong and saturated. This strong metal line system has detection of many low-ionization transitions, including O I, Si II, Al II, and C II. The intermediate-ionization transitions, Si III and Si IV, also exhibit strong absorption. The high-ionization transition, $\mathrm{N} \mathrm{v}$, however, is not detected, and $\mathrm{O}$ vI is not covered. Again, other than the possible line locking, we would not suspect that this system is intrinsic. We would classify this system as a 1-HIL system.

$Q 1107+4847\left(z_{\mathrm{em}}=3.000\right)$. - The radio source corresponding to this quasar is very weak $(0.48 \mathrm{mJy}$ at $4.95 \mathrm{GHz}$; Kuhn et al. 2001). It is detected within $10^{\prime \prime}$ of the optical source at $1.4 \mathrm{GHz}$ (NVSS). The X-ray-to-optical flux ratio is $\alpha_{\mathrm{ox}}=1.809$ (Kuhn et al. 2001 ).

$z_{\mathrm{abs}}=2.7243(\operatorname{class} A)$. - A C IV NAL was identified by Carballo et al. (1995). We also detected a Si IV NAL. Both MINFIT and the pixel-by-pixel method found partial coverage, with $C_{f}=0.49 \pm 0.04$ at the center of the strongest Si IV NAL component. We also find small $C_{f}$ values for the weaker components to the red, but those are subject to larger continuum fitting errors. The corresponding $C$ IV NAL also shows partial coverage in its strongest component at $\Delta v=-8 \mathrm{~km} \mathrm{~s}^{-1}$, with $C_{f}=0.70+0.17-0.15$. The other C IV components yield $C_{f}$ values consistent with 1 . There is a possibility that the $\mathrm{Si}$ IV $\lambda 1403$ profile is affected by absorption related to the feature at $-100 \mathrm{~km} \mathrm{~s}^{-1}$ in its window, which would affect our $C_{f}$ calculation. However, we note that partial coverage is also found for one of the C IV components in this system. The velocity plot of this system is presented in Figure 13.24. The Ly $\alpha$ and $\mathrm{Ly} \beta$ are strong and saturated. Very weak $\mathrm{C}$ II $\lambda 1335$ is detected at $\Delta v<0 \mathrm{~km} \mathrm{~s}^{-1}$, but not at $\Delta v>0 \mathrm{~km} \mathrm{~s}$. Also, at positive velocities, the $\mathrm{C}$ IV absorption is relatively stronger than the $\mathrm{Si}$ IV absorption. This system appears to have an ionization gradient with velocity. $\mathrm{N} v$ is not detected in a blend-free region of the spectrum. C IV, as well as Si IV NAL, shows evidence for partial coverage, although only in one component, so this is likely to be an intrinsic absorber. This system is classified as a 1-HIL system.

$Q 1330+0108\left(z_{\mathrm{em}}=3.510\right)$. - This quasar is listed in a catalog of high-luminosity quasars (Surdej et al. 1993). A radio source is not detected to a limit of $2.5 \mathrm{mJy}$ at $1.4 \mathrm{GHz}$ (NVSS).

$z_{\mathrm{abs}}=3.1148($ class $A)$. $-\mathrm{C}$ IV and $\mathrm{Si}$ IV NALs are identified in this system. The weaker component of the $\mathrm{C}$ IV NAL, at $\Delta v=$ $-17 \mathrm{~km} \mathrm{~s}^{-1}$, has a small coverage fraction, $C_{f}=0.45 \pm 0.10$, while the stronger one at $\Delta v=12 \mathrm{~km} \mathrm{~s}^{-1}$ has $C_{f}=0.89 \pm 0.10$. 
Although the latter differs from 1 by $<1 \sigma$, its profile shape at the bottom is reminiscent of a saturated profile that should be black for full coverage. The Si Iv NAL has a similar line profile to the $\mathrm{C}$ iv NAL, but it shows full coverage. The $\mathrm{S} / \mathrm{N}$ of this spectrum is low around both the $\mathrm{Si}$ IV and $\mathrm{C}$ IV doublets, leading to some uncertainties. The velocity plots for various transitions in this system are presented in Figure 13.25. Ly $\alpha$ is strong and saturated and extends far redward of the $\mathrm{C}$ Iv. Very weak $\mathrm{C}$ II $\lambda 1335$ is detected. The $\mathrm{N} v$ doublet suffers from blending in the forest but can be constrained not to be strong at the velocity of this absorber. This would therefore classify as a 1 -HIL system.

Q1334-0033 $\left(z_{\mathrm{em}}=2.801\right)$.- This LBQS quasar is identified at $850 \mu \mathrm{m}$ with $2.5 \pm 2.6 \mathrm{mJy}$ using the SCUBA array camera on the James Clerk Maxwell Telescope (JCMT; Priddey et al. 2003). We classify this quasar as radio-quiet because there is no radio source detection within $10^{\prime \prime}$ of the optical coordinate at $1.4 \mathrm{GHz}$ (NVSS).

$z_{\mathrm{abs}}=2.2010($ class $B)$. - Six overlapping C Iv components are detected within $\Delta v= \pm 100 \mathrm{~km} \mathrm{~s}^{-1}$ of each other. Five of these components show partial coverage, with large variation in the values with velocity. Although there is a weak component at $\Delta v=$ $114 \mathrm{~km} \mathrm{~s}^{-1}$ whose $C_{f}$ value is small and not consistent with 1 within $3 \sigma$, this is at the edge of the line profile and not very reliable. $C_{f}$ values of the other components are all consistent with 1.0 within $3 \sigma$. Therefore, we classify this as a class B system. The velocity plots for various transitions are presented in Figure 13.26. The Ly $\alpha$ is saturated and black in this region. There are no convincing detections of low-ionization transitions. The intermediate-ionization transitions, $\mathrm{Si}$ III and $\mathrm{Si}$ IV, may be detected, although they are confused by blends. $\mathrm{N} v$ is not detected and $\mathrm{O}$ vI is not covered. This would classify as a 1-HI system, with some uncertainty in whether intermediate-ionization transitions are detected.

$Q 1425+6039\left(z_{\mathrm{em}}=3.165\right)$. - The metallicity of the line-emitting BLR in this quasar was estimated to be $Z \sim 8 Z_{\odot}$ based on the emission-line flux ratios of $f(\mathrm{~N}$ v $\lambda 1240) / f(\mathrm{C}$ IV $\lambda 1549)(=8.4)$ and $f(\mathrm{~N}$ v $\lambda 1240) / f(\mathrm{He}$ II $\lambda 1640)(=5.1)$ (Dietrich \& Wilhelm-Erkens 2000).

$z_{\mathrm{abs}}=2.7699($ class $A)$.- The asymmetric line profile of this Si IV NAL is fitted well by two components. MINFIT determined small $C_{f}$ values for both components, $C_{f}=0.60 \pm 0.08$ and $0.52+0.28-0.22$. This system produces a sub-DLA line with $\log N_{\mathrm{H}_{\mathrm{I}}} \sim 19.37 \mathrm{~cm}^{-2}$, as well as various low- and intermediate-ionization metal lines such as O I $\lambda 1302, \mathrm{Si}$ II $\lambda 1190, \mathrm{Si}$ II $\lambda 1193, \mathrm{Si}$ II $\lambda 1527$, and $\mathrm{Si}$ III 21207 . At most, only a small amount of $\mathrm{C}$ IV absorption is detected, and $\mathrm{N}$ v and $\mathrm{O}$ vi absorption also appears not to be present. However, a system at $z=2.7728$, which is located only $230 \mathrm{~km} \mathrm{~s}^{-1}$ redward of this system, has high-ionization lines such as the $\mathrm{C}$ IV, $\mathrm{N}$ v, and $\mathrm{O}$ VI doublets. Although the $\mathrm{C}$ IV doublet in this system is black at its line center and thus has full coverage, $C_{f}=1$, these two systems could be physically related because of small velocity difference from each other. The velocity plots are presented in Figure 13.27 for various transitions. The coverage fraction analysis shows fairly convincing evidence for partial coverage in $\mathrm{Si}$ iv. However, it is unusual that this system is a sub-DLA, with strong low-ionization absorption and very little highionization absorption. We cannot reject an idea that an intrinsic system happens to blend with an intervening sub-DLA system. Ellison et al. (2002) have reported associated DLAs but interpret them as a population of galaxies clustering with the quasar host. This system, however, is at $v_{\text {shift }}=-29,800 \mathrm{~km} \mathrm{~s}^{-1}$, so in this case there would have to be a self-shielded neutral region as part of the intrinsic absorber. This system can be classified as 1-HIL, although it has an unusually low ionization state.

$Q 1548+0917\left(z_{\mathrm{em}}=2.749\right)$. - This quasar was discovered by Hazard et al. (1986). The Ly $\alpha$ emission line flux is $F(\operatorname{Ly} \alpha)=$ $1.41 \times 10^{-13} \mathrm{ergs} \mathrm{s}^{-1} \mathrm{~cm}^{-2}$, and the flux ratio between $\mathrm{C}$ Iv and Ly $\alpha$ is $0.45 \pm 0.04$ (Steidel et al. 1991). Steidel et al. (1991) discovered a companion galaxy located $4.8^{\prime \prime}$ from the quasar and $\sim 1000 \mathrm{~km} \mathrm{~s}^{-1}$ redshifted from the quasar. This galaxy is one of a few examples that have been discovered in the vicinity of "radio-quiet" quasars.

$z_{\mathrm{abs}}=2.3198$ (class A).--Sargent et al. (1989) found a $\mathrm{C}$ IV doublet from this sub-DLA system that has $\log N_{\mathrm{H}_{\mathrm{I}}} \sim 19.81$ (Dittmann \& Koeppen 1995). We detected C IV and Si Iv doublet lines. The C IV can be fitted with two components; however, for the blueward component, the doublet ratio is greater than 2 . This could be due to contamination of the $\mathrm{C}$ IV $\lambda 1548$ profile with the unknown blend at $\Delta v \sim-130 \mathrm{KM} \mathrm{s}^{-1}$. The redward component, however, shows $C_{f}=0.43 \pm 0.07$, which is convincing evidence for partial coverage assuming that it is not affected by blends. The Si IV doublet shows four components. The redward part has a line profile (inverted triangle) that suggests that a narrower and a broader component exist. The doublet ratio of Si IV is greater than 2 in some regions, so there is no evidence for partial coverage in that doublet. Although the bluest component of Si IV shows $C_{f}=0.18 \pm 0.08$, it is weak and subject to large continuum fitting errors. The velocity plot for this system is presented in Figure 13.28. The Ly $\alpha$ profile is damped. Many low-ionization lines that are covered in our spectrum (i.e., $\mathrm{O}$ I, Si II, Al II, C II) are detected, while the $\mathrm{N} v$ doublet is not. Although the coverage fraction of one of the $\mathrm{C}$ IV components leads us to classify this system as intrinsic, it seems unusual that an intrinsic system would have a damped Ly $\alpha$ profile. Any host galaxy absorption would be at a redshift much closer to that of the quasar, and not at the large velocity offset suggested for this system $\left(v_{\text {shift }} \sim-36,000 \mathrm{~km} \mathrm{~s}^{-1}\right)$. Again, we cannot reject an idea that an intrinsic system is simply blending with an unrelated intervening sub-DLA system. If this is indeed an intrinsic system, as our coverage fraction analysis has shown, we would classify it as a 1-HIL system. On the other hand, if this is an intervening system, $C$ IV $\lambda 1551$ would have to be blended with another line in order to produce an incorrect $C_{f}$ value.

$z_{\text {abs }}=2.6082$ (class B). - Two components are required to fit the absorption profile of $\mathrm{Si}$ iv in this system. The redder component, at $\Delta v=13 \mathrm{~km} \mathrm{~s}^{-1}$, shows apparent partial coverage, with $C_{f}=0.72+0.10-0.09$. However, the $\mathrm{S} / \mathrm{N}$ in this region is low, and we must consider the continuum level uncertainty. Nonetheless, the results from the profile fitting method and the pixel-by-pixel method are fairly consistent at the center of the component. The velocity plots for various transitions are presented in Figure 13.29. Similar line profiles are seen in several transitions such as Al II, Si II, Si III, C II, and C IV, although the C IV doublet does not show partial coverage. We did not fit the trough around $\Delta v=80 \mathrm{~km} \mathrm{~s}^{-1}$ because the doublet ratio between $\mathrm{Si}$ Iv $\lambda 1394$ and $\mathrm{Si}$ IV $\lambda 1403$ is larger than 2, implying that contamination by other lines has occurred. Ly $\alpha$ is very strong and wide, $\sim 500 \mathrm{~km} \mathrm{~s}^{-1}$, but is not fully black at some velocities. This system would classify as a 1-HIL system.

$z_{\mathrm{abs}}=2.6659($ class $A)$. - This very weak $\mathrm{C}_{\mathrm{IV}}$ system can be fitted with two components that are separated by $\sim 40 \mathrm{~km} \mathrm{~s}^{-1}$ from each other. The bluer component has a $C_{f}$ value that differs from 1 by more than $3 \sigma, C_{f}=0.29+0.15-0.12$, even if we consider uncertainty due to the error in the continuum level. The system is plotted in velocity space in Figure 13.30. C II and the very weak 
Si IV doublet are also identified. We do not find an $\mathrm{N} v$ doublet. Ly $\alpha$ is saturated at the center of the system. The evidence for this being an intrinsic system is just the partial coverage seen in the $\mathrm{C}$ IV doublet. We classify it as a 1-HIL system.

$z_{\mathrm{abs}}=2.6998($ class $A)$. - This is an associated NAL system whose velocity offset from the quasar is $v_{\text {shift }} \sim-4000 \mathrm{~km} \mathrm{~s} \mathrm{~s}^{-1}$. The $\mathrm{C}$ IV line is fitted with two components, the stronger of which shows partial coverage, and the weaker of which is consistent with full coverage. For the Si Iv line, we cannot derive a physical value of $C_{f}$, most likely due to a blend with the Si IV $\lambda 1394$ line. The velocity plots are presented in Figure 13.31. This system has a saturated Ly $\alpha$ line, which is black. Very weak $\mathrm{Si}$ II and $\mathrm{C}$ II absorption and strong Si III absorption are detected. As mentioned above, Si IV is detected, but the Si IV $\lambda 1394$ transition appears to be contaminated by a blend. Neither $\mathrm{N}$ v nor O vI is detected. The only evidence for an intrinsic nature for this system is the partial coverage for one $\mathrm{C}$ IV component. We classify it as a 1-HIL system.

Q1554+3749 $\left(z_{\mathrm{em}}=2.664\right)$. - This quasar was discovered by the Palomar Transit Grism Survey (Schneider et al. 1994). A radio source corresponding to the optical source was detected at $1.4 \mathrm{GHz}$ with a flux of $6.64 \mathrm{mJy}$. The radio-to-optical flux ratio is $\sim 12$, which classifies this quasar as radio-loud based on the criteria in Kellermann et al. $(1989,1994)$. We, however, classify the quasar as radio-quiet for the purpose of our study because the other radio-loud quasars in our sample have much larger values, $\mathcal{R}>130$.

$z_{\mathrm{abs}}=2.3777$ (class $A$ ).-This asymmetric Si IV NAL is adequately fitted with two components. The broader component at $\Delta v=6 \mathrm{~km} \mathrm{~s}^{-1}$ shows partial coverage, but the narrower component has a $C_{f}$ value consistent with 1 . The velocity plot of this system is presented in Figure 13.32. Low-ionization lines, such as C II $\lambda 1335$ and Si II $\lambda 1260$, are detected, although the latter could be blended with other lines since it is usually weak compared to $\mathrm{C}_{\text {II }} \lambda 1335$. In fact, these low-ionization transitions show weaker outlying clouds at $\Delta v \sim 80-130 \mathrm{~km} \mathrm{~s}^{-1}$, which are very strong in Si III $\lambda 1207$ and apparent in the Si IV $\lambda 1394$ profile, but not detected in the Si IV $\lambda 1403$ profile. C IV is not covered. We do not see an $\mathrm{N} v$ in this system. This system classifies as 1 -HIL.

HS $1700+6416\left(z_{\mathrm{em}}=2.722\right)$. - This quasar was discovered in the course of the Hamburg quasar survey as one of the most luminous high- $z$ quasars. The ROSAT image of the quasar field shows two bright X-ray clusters, whose X-ray emission extends to the line of sight of the quasar (Reimers et al. 1997). The high luminosity of the quasar could be amplified by the gravitational lensing effects through these two clusters, one of which is identified as Abell 2246.

$z_{\text {abs }}=2.4330$ (class $\left.B\right)$. - Tripp et al. (1997) noticed a prominent complex of C IV NALs at $z_{\text {abs }}=2.432-2.441$, which shows line locking. This system has six narrow components, but $C_{f}$ cannot be derived directly for any of them because of the superposition of the $\mathrm{C}_{\text {IV }} \lambda 1551$ member with the $\mathrm{C}$ IV $\lambda 1548$ member of the $z_{\mathrm{abs}}=2.4394$ system. In order to evaluate the coverage fraction in this blended system, we first fitted the $\mathrm{C}$ IV $\lambda 1548$ transition from the $z_{\text {abs }}=2.4330$ system and the C IV $\lambda 1551$ transition from the $z_{\text {abs }}=2.4394$ system, assuming $C_{f}=1$. Then we applied the resulting column densities and Doppler parameters to model the expectation for the blended region of the spectrum. We find that this model is almost consistent with the data, which implies full coverage for these systems. The velocity plots for various transitions are presented in Figure 13.33. The Ly $\alpha$ profile is very strong and black over the velocity range of interest for this system, but the normalized flux recovers to 1 between this and the $z_{\text {abs }}=$ 2.4394 system. The low-ionization transition, $\mathrm{C}_{\text {II }} \lambda 1335$, is clearly detected, with possible detections of Si II $\lambda 1260$ and Al II $\lambda 1671$. $\mathrm{Si}$ III and the Si IV doublet are detected, but the $\mathrm{Si}$ IV $\lambda 1403$ line is contaminated by a blend. $\mathrm{N} v$ is not detected and $\mathrm{O}$ VI is not covered. The line locking for this system, which appears as a precise match for several components, is the only evidence for an intrinsic origin. We would classify this system as a 1-HIL system.

$z_{\mathrm{abs}}=2.4394$ (class $\left.B\right)$. - This C IV NAL has seven components. All but the reddest three components are affected by blending (in $C$ IV 21548 ) with $C$ IV $\lambda 1551$ from the $z_{\text {abs }}=2.4330$ system. Although we cannot evaluate $C_{f}$ values for this heavily blended system, we expect that all components have full coverage, based on the analysis described above for the $z_{\text {abs }}=2.4330$ system. The velocity plots for various transitions are presented in Figure 13.34. The strong Ly $\alpha$ absorption is black for this system, but it lacks low-ionization absorption. Si III and Si IV are detected, but the Si IV doublet has an inconsistent doublet ratio, with Si IV $\lambda 1394$ being relatively too strong. $\mathrm{N} v$ is not detected and $\mathrm{O}$ vI is not covered. Because of the line locking of this system with the $z_{\mathrm{abs}}=2.4330$ system, we classify it as a probable intrinsic system. We note, however, that it probably does not have partial coverage and that it is otherwise indistinguishable from an intervening system. It would be classified as 1-HI if it is intrinsic.

$z_{\mathrm{abs}}=2.7125(\operatorname{class} A)$. - This associated system is one of the most reliable intrinsic NAL systems in our sample. Tripp et al. (1997) noted that it could only partially cover the quasar flux source by their apparent column density profile analysis of the $\mathrm{N} v$ doublet, using a lower resolution spectrum $\left(\mathrm{FWHM} \sim 20 \mathrm{~km} \mathrm{~s}^{-1}\right.$ ). In our spectrum, both $\mathrm{C}$ IV and $\mathrm{N} v$ lines are detected and both doublets show evidence for partial coverage. The asymmetric line profiles require multiple components for an adequate fit. The velocity plot of this system is presented in Figure 13.35. It is striking that the Ly $\alpha$ line is weaker than even $\mathrm{N} v \lambda 1239$ for this system. However, we note that if the coverage fraction for $\operatorname{Ly} \alpha$ is $C_{f} \sim 0.4$, consistent with the value determined from $\mathrm{N}$, we would see a saturated profile with minimum normalized flux of 0.6 . Low- and intermediate-ionization transitions are not detected for this system. $\mathrm{O}$ VI may be detected, but the $\mathrm{O}$ vI $\lambda 1038$ line is affected by a blend, and the $\mathrm{O}$ VI $\lambda 1032$ line probably is as well. If most of the apparent $\mathrm{O}$ VI absorption is real, $\mathrm{O}$ VI would be much stronger than the $\mathrm{N}$ v and $\mathrm{C}$ IV lines. This is a $2-\mathrm{H}$ system.

$z_{\mathrm{abs}}=2.7164$ (class $\left.A\right)$. - This is an associated system, separated by only $\sim 300 \mathrm{~km} \mathrm{~s}^{-1}$ from the $z_{\text {abs }}=2.7125 \mathrm{system}$. We fit the very weak line profile of the $\mathrm{N} v$ doublet with one component. The covering factor is estimated to be $0.39+0.09-0.08$. The pixelby-pixel method yields a similar result around line center. The system is plotted in velocity space in Figure 13.36 . While no lowand intermediate-ionization transitions are identified, some $\mathrm{O}$ vi absorption is probably detected in the Ly $\alpha$ forest. C IV $\lambda 1548$ is detected but is even weaker than the $\mathrm{N}$ v. Ly $\alpha$ is very weak, with almost no detection, which would be unusual for an intervening system. This intrinsic NAL is classified as a 2-H system.

HS 1946+ $7658\left(z_{\mathrm{em}}=3.051\right)$.- - This quasar was discovered by Hagen et al. (1992), using objective prism observations at the $80 \mathrm{~cm}$ Calar Alto Schmidt Telescope. Kuhn et al. (1995) obtained radio and X-ray data of the quasar and confirmed that its spectral energy distribution is generally similar to those of low- $z(z \sim 1)$ quasars in Elvis et al. (1994).

$z_{\mathrm{abs}}=2.8928$ (class $\left.A\right)$. - This system was identified by Tripp et al. (1996). A C IV NAL with two components is detected in our spectrum. We find $C_{f}=0.59+0.09-0.08$ for the redward component, but the blueward component has $C_{f}$ consistent with 1 . Figure 13.37 presents the velocity plots for various transitions. The Ly $\alpha$ profile is black and centered roughly on the two C IV 
components. Low-ionization absorption is possibly detected as very weak $\mathrm{C}$ II $\lambda 1335$. A weak Si IV doublet is detected for the redder component, but not for the bluer. $\mathrm{N} v$ is not detected, but we cannot tell if weak $\mathrm{O}$ v is detected or not because of blending in the Ly $\alpha$ forest. This system, if intrinsic, is probably a 1-HIL system.

$z_{\mathrm{abs}}=3.0385$ (class $\left.A\right)$. - In this associated system, we fit the weak C Iv NAL with three components. One of them, at $\Delta v=-3 \mathrm{~km} \mathrm{~s}^{-1}$, shows partial coverage, $C_{f} \sim 0.18$, by both the pixel-by-pixel and fitting methods. The other components have $C_{f}$ consistent with 1 . The velocity plot of this system is presented in Figure 13.38. Ly $\alpha$ is saturated and black, but not very strong compared to most other systems. $\mathrm{Ly} \beta$ is not saturated. No low- or intermediate-ionization transitions are detected to sensitive limits. $\mathrm{N} \mathrm{v}$ is not detected, and $\mathrm{O}$ VI is again contaminated by Ly $\alpha$ forest lines so that no judgment can be made. This is probably a 1-H system.

$z_{\text {abs }}=3.0497$ (class A).-Tripp et al. (1996) studied this associated system in detail and from photoionization modeling concluded that it is not likely to be intrinsic. The system is separated by only $\sim 800 \mathrm{~km} \mathrm{~s}^{-1}$ from the class A system at $z_{\text {abs }}=3.0385$. We identified $\mathrm{N} v, \mathrm{Si}$ Iv, and C Iv NALs. The N v NAL is classified as class A because one of its components shows partial coverage. On the other hand, C IV and Si IV NALs have full coverage because they are black at line centers. The velocity plot of this system is presented in Figure 13.39. The Ly $\alpha$ profile is black and about the same equivalent width as $\mathrm{C}$ Iv. We attribute this to a similar velocity spread for multiple saturated components in both transitions. The low-ionization transitions, $\mathrm{Si}$ II and $\mathrm{C}$ II, are detected, but $\mathrm{O}$ I is not. $\mathrm{Si}$ III, $\mathrm{C}$ III, and Si IV are very strong, with broad, black line profiles. It is again difficult to assess whether $\mathrm{O}$ VI absorption is detected, although alignment of one component in the $\mathrm{O}$ vI $\lambda 1038$ profile with other transitions suggests that it is. High- and low-ionization transitions, such as $\mathrm{N} v$ and $\mathrm{Si}$ II, show good alignment with each other. This is clearly a multiphase absorber. Our assessment that it might be intrinsic hinges on determination of $C_{f}$ in one of the $\mathrm{N} v$ components. We would classify this system as a 1-HIL system.

Adam, G. 1985, A\&AS, 61, 225

Anderson, S. F., Weymann, R. J., Foltz, C. B., \& Chaffee, F. H. 1987, AJ, 94, 278

Arav, N., Brotherton, M. S., Becker, R. H., Gregg, M. D., White, R. L., Price, T., \& Hack, W. 2001a, ApJ, 546, 140

Arav, N., Li, Z.-Y., \& Begelman, M. C. 1994, ApJ, 432, 62

Arav, N., et al. 2001b, ApJ, 561, 118

Balsara, D. S., \& Krolik, J. H. 1993, ApJ, 402, 109

Barlow, T. A., \& Sargent, W. L. W. 1997, AJ, 113, 136

Barthel, P. D., Tytler, D. R., \& Vestergaard, M. 1997, in ASP Conf. Ser. 128, Mass Ejection from Active Galactic Nuclei, ed. N. Arav, I. Shlosman, \& R. J. Weymann (San Francisco: ASP), 48

Barvainis, R., \& Ivison, R. 2002, ApJ, 571, 712

Bechtold, J., et al. 2003, ApJ, 588, 119

Becker, R. H., White, R. L., \& Edwards, A. L. 1991, ApJS, 75, 1

Bergeron, J., et al. 1994, ApJ, 436, 33

Blandford, R. D., \& Payne, D. G. 1982, MNRAS, 199, 883

Brotherton, M. S., Wills, B. J., Steidel, C. C., \& Sargent, W. L. W. 1994, ApJ, 423, 131

Burles, S., \& Tytler, D. 1998, ApJ, 507, 732

Carballo, R., Barcons, X., \& Webb, J. K. 1995, AJ, 109, 1531

Carswell, R. F., Williams, R. E., Beaver, E. A., Harms, R., \& Strittmatter, P. A. 1975, ApJ, 195, 269

Chelouche, D., \& Netzer, H. 2005, ApJ, 625, 95

Chen, J.-S., Morton, D. C., Peterson, B. A., Wright, A. E., \& Jauncey, D. L. 1981, MNRAS, 196, 715

Chiang, J., \& Murray, N. 1996, ApJ, 466, 704

Churchill, C. W., \& Vogt, S. S. 2001, AJ, 122, 679

Condon, J. J., Cotton, W. D., Greisen, E. W., Yin, Q. F., Perley, R. A., Taylor, G. B., \& Broderick, J. J. 1998, AJ, 115, 1693

Constantin, A., Shields, J. C., Hamann, F., Foltz, C. B., \& Chaffee, F. H. 2002, ApJ, 565, 50

Corbin, M. R. 1990, ApJ, 357, 346

Crenshaw, D. M., Kraemer, S. B., Gabel, J. R., Schmitt, H. R., Filippenko, A. V., Ho, L. C., Shields, J. C., \& Turner, T. J. 2004, ApJ, 612, 152

Crenshaw, D. M., Maran, S. M., \& Mushotzky, R. F. 1998, ApJ, 496, 797

de Kool, M., Korista, K. T., \& Arav, N. 2002, ApJ, 580, 54

Dietrich, M., \& Wilhelm-Erkens, U. 2000, A\&A, 354, 17

Dittmann, O. J., \& Koeppen, J. 1995, A\&A, 297, 671

D’Odorico, V., Cristiani, S., Romano, D., Granato, G. L., \& Danese, L. 2004, MNRAS, 351, 976

Ellison, E., Yee, H. K. C., \& Green, R. F. 1991, ApJ, 371, 49

Ellison, S. L., Yan, L., Hook, I. M., Pettini, M., Wall, J. V., \& Shaver, P. 2002, A\&A, 383, 91

Elvis, M. 2000, ApJ, 545, 63

Elvis, M., et al. 1994, ApJS, 95, 1

Emmering, R. T., Blandford, R. D., \& Shlosman, I. 1992, ApJ, 385, 460

Everett, J. E. 2005, ApJ, 631, 689

Foltz, C. B., Weymann, R. J., Peterson, B. P., Sun, L., Malkan, M. A., \& Chaffee, F. H. 1986, ApJ, 307, 504 (F86)

Gabel, J. R., Arav, N., \& Kim, T.-S. 2006, ApJ, 646, 742

Ganguly, R., Bond, N. A., Charlton, J. C., Eracleous, M., Brandt, W. N., \& Churchill, C. W. 2001, ApJ, 549, 133 (G01)
Ganguly, R., Eracleous, M., Charlton, J. C., \& Churchill, C. W. 1999, AJ, 117, 2594 (G99)

Ganguly, R., Masiero, J., Charlton, J. C., \& Sembach, K. R. 2003, ApJ, 598, 922

Granato, G. L., De Zotti, G., Silva, L., Bressan, A., \& Danese, L. 2004, ApJ, 600,580

Grevesse, N., \& Anders, E. 1989, in AIP Conf. Proc. 183, Cosmic Abundances of Matter, ed. C. I. Waddington (New York: AIP), 1

Griffith, M. R., Wright, A. E., Burke, B. F., \& Ekers, P. D. 1995, ApJS, 97, 347

Hagen, H.-J., Groote, D., Engels, D., \& Reimers, D. 1995, A\&AS, 111, 195

Hagen, H.-J., et al. 1992, A\&A, 253, L5

Hall, P. B., et al. 2002, ApJS, 141, 267

Hamann, F. 1997, ApJS, 109, 279

1998, ApJ, 500, 798

Hamann, F., Barlow, T. A., \& Junkkarinen, V. 1997a, ApJ, 478, 87

Hamann, F., Barlow, T. A., Junkkarinen, V., \& Burbidge, E. M. 1997b, ApJ, 478,80

Hamann, F., Korista, K. T., \& Morris, S. L. 1993, ApJ, 415, 541

Hamann, F., \& Sabra, B. 2004, in ASP Conf. Ser. 311, AGN Physics with the Sloan Digital Sky Survey, ed. G. T. Richards \& P. B. Hall (San Francisco: ASP), 203

Hazard, C., Morton, D. C., McMahon, R. G., Sargent, W. L. W., \& Terlevich, R. 1986, MNRAS, 223, 87

Hewitt, A., \& Burbidge, G. 1987, ApJS, 63, 1

Hook, I. M., McMahon, R. G., Irwin, M. J., \& Hazard, C. 1996, MNRAS, 282, 1274

Isaak, K. G., Priddey, R. S., McMahon, R. G., Omont, A., Peroux, C., Sharp, R. G., \& Withington, S. 2002, MNRAS, 329, 149

Kaspi, S., Netzer, H., Chelouche, D., George, I. M., Nandra, K., \& Turner, T. J. 2004, ApJ, 611, 68

Kellermann, K. I., Sramek, R., Schmidt, M., Shaffer, D. B., \& Green, R. 1989, AJ, 98, 1195

Kellermann, K. I., Sramek, R. A., Schmidt, M., Green, R. F., \& Shaffer, D. B. 1994, AJ, 108, 1163

Kirkman, D., et al. 2005, MNRAS, 360, 1373

Konigl, A., \& Kartje, J. F. 1994, ApJ, 434, 446

Kormann, R., Schneider, P., \& Bartelmann, M. 1994, A\&A, 286, 357

Krolik, J. H., \& Kriss, G. A. 1995, ApJ, 447, 512 2001, ApJ, 561, 684

Kuhn, O., Bechtold, J., Cutri, R., Elvis, M., \& Rieke, M. 1995, ApJ, 438, 643 Kuhn, O., Elvis, M., Bechtold, J., \& Elston, R. 2001, ApJS, 136, 225

Marziani, P., Sulentic, J. W., Dultzin-Hacyan, D., Calvani, M., \& Moles, M. 1996, ApJS, 104, 37

Misawa, T., Eracleous, M., Charlton, J. C., \& Tajitsu, A. 2005, ApJ, 629, 115 (M05)

Misawa, T., Tytler, D., Iye, M., Storrie-Lombardi, L. J., Suzuki, N., \& Wolfe, A. M. 2002, AJ, 123, 1847

Misawa, T., Yamada, T., Takada-Hidai, M., Wang, Y., Kashikawa, N., Iye, M., \& Tanaka, I. 2003, AJ, 125, 1336

Monet, D., et al. 1998, USNO-A V2.0, A Catalog of Astrometric Standards (Flagstaff: USNO)

. 2003, AJ, 125, 984

Murray, N., \& Chiang, J. 1997, ApJ, 474, 91 
Murray, N., Chiang, J., Grossman, S. A., \& Voit, G. M. 1995, ApJ, 451, 498 Narayanan, D., Hamann, F., Barlow, T., Burbidge, E. M., Cohen, R. D., Junkkaribeb, V., \& Lyons, R. 2004, ApJ, 601, 715

Neff, S. G., \& Hutchings, J. B. 1990, AJ, 100, 1441

Netzer, H., et al. 2003, ApJ, 599, 933

Oke, J. B., \& Schild, R. E. 1970, ApJ, 161, 1015

O’Meara, J. M., Tytler, D., Kirkman, D., Suzuki, N., Prochaska, J. X., Lubin, D., \& Wolfe, A. M. 2001, ApJ, 552, 718

Osmer, P. S., \& Smith, M. G. 1976, ApJ, 210, 267

Page, K. L., Turner, M. J. L., Reeves, J. N., O’Brien, P. T., \& Sembay, S. 2003, MNRAS, 338, 1004

Petitjean, P., Rauch, M., \& Carswell, R. F. 1994, A\&A, 291, 29

Petitjean, P., \& Srianand, R. 1999, A\&A, 345, 73

Press, W. H., Rybicki, G. B., \& Schneider, D. P. 1993, ApJ, 414, 64

Priddey, R. S., Isaak, K. G., McMahon, R. G., \& Omont, A. 2003, MNRAS, 339, 1183

Proga, D., Stone, J. M., \& Kallman, T. R. 2000, ApJ, 543, 686

Reichard, T. A., et al. 2003, AJ, 126, 2594

Reimers, D., Rodriguez-Pascual, P., Hagen, H.-J., \& Wisotzki, L. 1995, A\&A, 293, L21

Reimers, D., Toussaint, F., Hagen, H.-J., Hippelein, H., \& Meisenheimer, K. 1997, A\&A, 326, 489

Richards, G. T. 2001, ApJS, 133, 53

Richards, G. T., Laurent-Muehleisen, S. A., Becker, R. H., \& York, D. G. 2001, ApJ, 547, 635

Richards, G. T., York, D. G., Yanny, B., Kollgaard, R. I., Laurent-Muehleisen, S. A., \& Vanden Berk, D. E. 1999, ApJ, 513, 576

Sabra, B. M., \& Hamann, F. 2005, preprint (astro-ph/0509421)

Sargent, W. L. W., Boksenberg, A., \& Steidel, C. C. 1988, ApJS, 68, 539

Sargent, W. L. W., Steidel, C. C., \& Boksenberg, A. 1989, ApJS, 69, 703

Scannapieco, E., \& Oh, S. P. 2004, ApJ, 608, 62

Schmidt, M., \& Green, R. 1983, ApJ, 269, 352
Schneider, D. P., Schmidt, M., \& Gunn, J. E. 1994, AJ, 107, 1245

Scott, J. E., et al. 2004, ApJS, 152, 1

Shakura, N. I., \& Sunyaev, R. A. 1973, A\&A, 24, 337

Smith, M. G., \& Wright, A. E. 1980, MNRAS, 191, 871

Sprayberry, D., \& Foltz, C. B. 1992, ApJ, 390, 39

Springel, V., Di Matteo, T., \& Hernquist, L. 2005, ApJ, 620, L79

Srianand, R., \& Petitjean, P. 2000, A\&A, 357, 414

Steidel, C. C. 1990, ApJS, 72, 1

Steidel, C. C., Dickinson, M., \& Sargent, W. L. W. 1991, AJ, 101, 1187

Steidel, C. C., \& Sargent, W. L. W. 1991, ApJ, 382, 433

Storrie-Lombardi, L. J., McMahon, R. G., Irwin, M. J., \& Hazard, C. 1996, ApJ, 468, 121

Sulentic, J. W., Marziani, P., Dultzin-Hacyan, D., Calvani, M., \& Moles, M. 1995, ApJ, 445, L85

Surdej, J., et al. 1993, AJ, 105, 2064

Tripp, T. M., Lu, L., \& Savage, B. D. 1996, ApJS, 102, 239 1997, ApJS, 112, 1

Tytler, D., \& Fan, X.-M. 1992, ApJS, 79, 1

Vanden Berk, D. E., et al. 2001, AJ, 122, 549

Véron-Cetty, M.-P., \& Véron, P. 2003, A\&A, 412, 399

Vestergaard, M. 2003, ApJ, 599, 116

Wampler, E. J., Chugai, N. N., \& Petitjean, P. 1995, ApJ, 443, 586

Weymann, R. J., Morris, S. L., Foltz, C. B., \& Hewett, P. C. 1991, ApJ, 373, 23

Weymann, R. J., Williams, R. E., Peterson, B. M., \& Turnshek, D. A. 1979, ApJ, 234, 33

Wise, J. H., Eracleous, M., Charlton, J. C., \& Ganguly, R. 2004, ApJ, 613, 129

Wold, M., Lacy, M., Lilie, P. B., \& Serjeant, S. 2000, MNRAS, 316, 267

Yee, H. K., \& Ellison, E. 1993, ApJ, 411, 43

Yee, H. K., \& Green, R. F. 1987, ApJ, 319, 28

Young, P., Sargent, W. L. W., \& Boksenberg, A. 1982, ApJS, 48, 455

Yuan, Q., Green, R. F., Brotherton, M., Tripp, T. M., Kaiser, M. E., \& Kriss, G. A. 2002, ApJ, 575, 687 